\title{
Serotonin and Parkinson
}

Citation for published version (APA):

Scholtissen, B. (2005). Serotonin and Parkinson. [Doctoral Thesis, Maastricht University]. NeuroPsych Publishers. https://doi.org/10.26481/dis.20051102bs

Document status and date:

Published: 01/01/2005

DOI:

10.26481/dis.20051102bs

Document Version:

Publisher's PDF, also known as Version of record

\section{Please check the document version of this publication:}

- A submitted manuscript is the version of the article upon submission and before peer-review. There can be important differences between the submitted version and the official published version of record.

People interested in the research are advised to contact the author for the final version of the publication, or visit the DOI to the publisher's website.

- The final author version and the galley proof are versions of the publication after peer review.

- The final published version features the final layout of the paper including the volume, issue and page numbers.

Link to publication

\footnotetext{
General rights rights.

- You may freely distribute the URL identifying the publication in the public portal. please follow below link for the End User Agreement:

www.umlib.nl/taverne-license

Take down policy

If you believe that this document breaches copyright please contact us at:

repository@maastrichtuniversity.nl

providing details and we will investigate your claim.
}

Copyright and moral rights for the publications made accessible in the public portal are retained by the authors and/or other copyright owners and it is a condition of accessing publications that users recognise and abide by the legal requirements associated with these

- Users may download and print one copy of any publication from the public portal for the purpose of private study or research.

- You may not further distribute the material or use it for any profit-making activity or commercial gain

If the publication is distributed under the terms of Article $25 \mathrm{fa}$ of the Dutch Copyright Act, indicated by the "Taverne" license above, 


\section{Serotonin and Parkinson}

Neuropsych Publishers Maastricht, the Netherlands 
(c) Bart Scholtissen, Maastricht 2005

design / Katemingk grafische dienstvertening, Maastricht

cover intage / paintings by Carla Brandts

print | Drukkerii Gijsemberg, Maasmechelen

publisher / Neufopsych Publishers, Maastricht University, PO Box 616, NL-6200 MD Maastricht

isbon | 90-75579-24-9

Neuropsych Publishers is a nonprofit organization, which aims at promoting the science of 'Brain and Behaviour' and improving the application of the products of this science in health sare and eduration. Neuropsych Publishers accomplishes these aims by publishing books, dlissertations, and other products of scientific activity, by disseminating educational materials and publication of tests, assessment scales and other psychometric instruments in the field of Nouropsychology. Neuropsychiatry, and other areas within the domain of Brain and Behawiour.

All rights are reserved. No part of this book may be reproduced or transmitted in any form or by any means, without written permission from the author or, when appropriate, the publisher of the apticle. 


\title{
Serotonin and Parkinson
}

\author{
PROEFSCHRIFT
}

ter verkrijging van de graad van doctor aan de Universiteit Maastricht

op gezag van de Rector Magnificus, Prof. mr. G.P.M.F. Mols

volgens het besluit van het College van Decanen,

in het openbaar te verdedigen

op woensdag 2 november 2005 om 14.00 uur

door

\section{Bart Scholtissen}

geboren op 7 januari 1978 te Heerlen 


\section{Promotores}

Prof. dr. F.R.J. Verhey

Prof, dr. H.W.M. Steinbusch

\section{Co-promatores}

Dr. A.F.G. Leentjens

Dr. FW. Vreeling

\section{Beoordelingscommissie}

Prof. dr. J.J. Jolles (voorzitter)

Dr. A. Blokland

Dr. P.J. Koehler

Em. Prof. dr. H.M. van Praag

Prof. dr. W. Riedel

The research presented in thit thesis was performed at the Mastricht Brain \& Eehavilour linstitule. andisd the Department of Psychiatry \& Neuropsychology, Maastricht University.

The publication of this thesis was financially supported by:

Medtronic, Nowartis, Glaxo5mithkme, Parkinson patiëntenwerenigging, Pfizer, Vam Leersim KNAW Foundation, Lundbeck instyaeneca, Sanofi-Aventis. 
If you never try, you never know... 


\section{Contents}

1 Introduction 11

2 Serotonergic mechanisms in Parkinson's Disease

Opposing results from preclinical and clinical data

21

3 Functional investigations into the role of dopamine and serotonin in partial bilateral striatal 6 -hydroxydopamine lesioned rats

4 Recovery after a partial bilateral striatal 6-hydroxydopamine lesion in rats 61

5 Effects of acute tryptophan depletion on cognition, memory, and motor performance in Parkinson's Disease 71

6 The serotonergic hypothesis for depression in Parkinson's Disease An experimental approach 87

7 Challenging the serotonergic system in Parkinson's Disease patients Effects on cognition, memory, motor performance and mood

103

8 The manual tracking task for tremor assessment A new task for investigating manual tracking movement in $\mathrm{PD}$ 121

9 General discussion 133

10 Summary 151

11 Samenvatting 157

12 Dankwoord 163

13 Publications 171

14 Curriculum vitae 


\section{Introduction}

\section{Parkinson's disease}

In 1817 a physician by the name of James Parkinson (1755-1824) published a monograph in which he described 6 patients, who all suffered from a disease then known as "paralysis agitans", also named 'the shaking palsy" 111. Written accounts of this "shaking disease' date back by approximately 4500 years when 'kampavata' (literally meaning: 'shaking rigidity') was described in the ayurveda writings. Since then several persons, among which Galen (131-201), have reported symptoms of what is nowadays known as Parkinson's disease (PD). In the writings of Shakespeare indications of PD may be found. See for instance the following citation from Henry Vl: "why dost thou quiver man? It is the palsy, and not fear, that provokes me". From these recordings of PD it might be clear that PD certainly is no "modern" disease that has only been around for the last decades.

$\mathrm{PD}$ is one of the most prevalent neuropsychiatric diseases, affecting approximately 75-175 per 100.000 people in the general population. The most frequently described symptoms are tremor, rigidity and bradykinesia. These are all motor symptoms, which find their pathophysiological origin in the degeneration of dopamine producing neurons in the substantia nigra pars compacta, an area in the mesencephalic part of the brain [21. These neurons project via the niligrostriatal tract to the striatum where they modulate the activity of the direct and indirect pathway [3]. The net result of this action is the reduction of GABA-ergic output to the thallamus, which results in a higher glutamatergic excitation of the cortex wia the cortico-thalamic pathway. In case of PD there is a loss of dopaminergic input from the substantia nigra pars compacta. This results in less inhibition of the direct pathway and more excitation of the indirect pathway in the basal ganglia. This in turn, results in less output to the thalamus and cortex, resulting in the symptoms which are so typical for PD (Figure 1).

Although PD was, and is, often described as a motor disease with familiar symp toms such as tremor, rigidity, and bradykinesia, this is not the whole story. Besides motor symptoms; cognitive and psychiatric symptoms such as depression and hallucina- 
tions are also frequently reported by patients. This is one of the reasons why PD should be seen as a neuropsychiatric instead of solely a neurological disease. Furthermore, it is known that other neurotransmitter systems than the dopamine system, such as the serotonin, horadrenalin, and cholinergic system are also involved in the pathophysiology of the disease. This thesis is mainly concerned with the role of serotonin in PD.

\section{Serotonin}

Serotonin (5-hydroxytryptophan or 5-HT) is a monoamine which is involved in a number of processes in brain and body. Serotonin is most commonly known from its role in the pathogenesis of depression $|4|$. This is one of the links between serotonin and PD. Depression is one of the most common comorbid psychiatric disorders in PD. The incidence of depression in PD ranges from $2-70 \%$, depending on the population test$\mathrm{ed}_{\mathrm{s}}$ and the methods used for testing. Generally, a mean percentage of $25-40 \%$ is used in literature $|5,6|$. Motor, cognitive and affective symptoms are primarily related to a decreased dopaminergic activity, but serotonergic mechanisms are most likely also involved. Reduction of postmortem serotonin levels in brains of PD patients $[7,8]$, a loss of neurons in the dorsal raphe $|9|$, and decreased levels of 5-hydroxyindole acid (5HIAA) in the cerebrospinal fluid (CSF) [10-13] point in this direction. This decreased serotonergic activity is thought to be correlated with the severity of motor symptoms [14]. It is unclear whether this decrease in serotonergic activity reflects a primary degenerative mechanism, or a physiological compensation mechanism. The precise role of a decreased serotonergic activity for motor symptoms, cognition, and affect remains unclear. From animal research it is known that serotonin and dopamine are able to inhibit each others activity in the striatum $|15|$. Furthermore, reduced serotonergic activity also influences cognitive functioning $|16-19|$.

\section{Aims of the thesis}

The main aim of this thesis is to gain a better insight into the role of the serotonergic neurotransmitter system in the pathophysiology and symptomatology of PD. The research described in this thesis is based on findings from both clinical and animal research. The overall idea behind the studies described in the present thesis all find their origin in the aspiration and necessity to link preclinical and clinical research. Both fields of research, unfortunately, often operate isolated from each other, and the ultimate goal: gaining knowledge in order to improve or cure a disease, is sometimes lost out of sight. Both fields should ideally benefit from results realized in the other field. 
Although this aim was not always within easy reach, this thesis tries to bridge this gap, by combining clinical and preclinical research on the topic of PD. Aglainst this back ground, the main research questions of this thesis are:

1. Is there a link between predinical and dinical research regarding investigations of functional interventions of the serotonergic neurotransmitter system in PD?

2. Can an animal model of PD provide useful information for the clinical practice?

3. Does an acute functional intervention of the serotonergic neurotransmitter system lead to observable changes in motor, cognition, and mood in patients suffering from $P D$ ?

\section{Outline of the thesis}

Chapter 2 provides an overview of functional serotonergic interventions in both animal models of PD and human PD. Over the last decades a lot of research has been done to further elucidate the exact role of serotonin in PD. There are two possible options of in. vestigating this matter. One is creating an animal model that mimics certain aspects of the human disease, and the other is directly intervening in serotonin levels in patients suffering from $P D$, and measuring the effects of this intervention by neuroimaging studies or behavioral and neuropsychological assessment. Since both lines of research have added knowledge to our understanding of serotonin and PD, the review will address both these fields. In both fields attention will be focused on functional interventions of the serotonergic neurotransmitter system.

Chapter 3 and 4 will deal with preclinical research into the role of serotonin in Parkin. son's Disease. Chapter 3 will describe behavioral research performed in an animal mod. el of PD, the partial bilateral 6-hydroxydopamine (6-OHDA) model. 6-OHDA is a neurotoxin which selectively lesions mono-aminergic fibers. When given in combination with desimipramine, 6-OHDA will only lesion dopaminergic fibers. Injecting 6-OHDA into the striatum of, for instance, rats produces an animal model which mimics certain motor symptoms of clinical PD. Chapter 3 describes the results of behavioral observations of the effects of different serotonergic and dopaminergic substances on reaction time responding in rats with partial bilateral 6-OHDA lesions. Chapter 4 will go into the second part of this experiment and will describe, in a qualitative way, immunohistochemi* cal outcomes of the study.

Chapter 5 to 8 report on the clinical part of the research. Chapter 5 will go into one widlely used method of experimentally influencing the serotonergic system, the method 


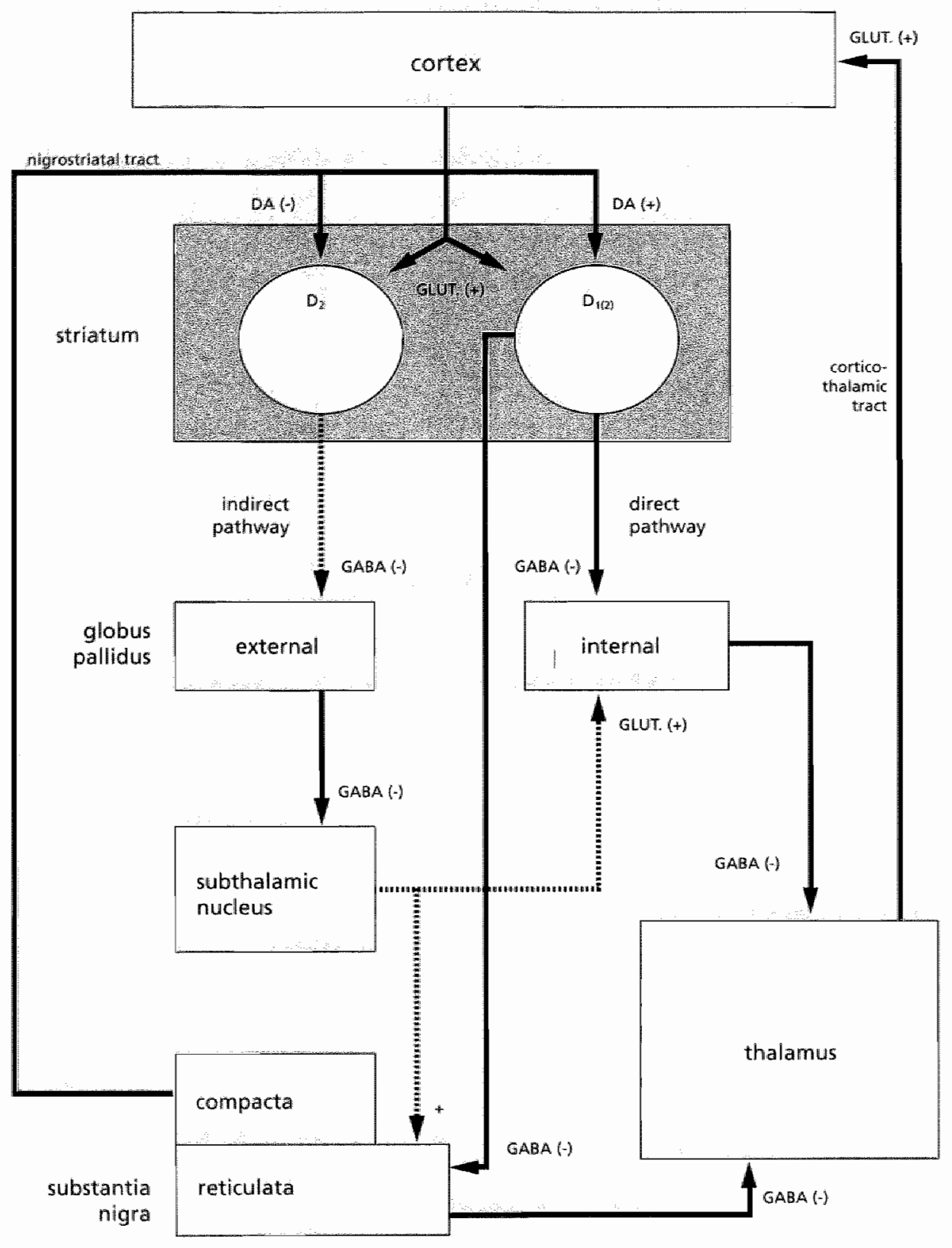

Figure 1. Schematic owerview of basal ganglia connections in the healthy situation (A) and in PD (B).

Pathway activity: dashed lines indicate low activity, thin solid lines indicate normal activity, and thick solid lines indicate high activity. 


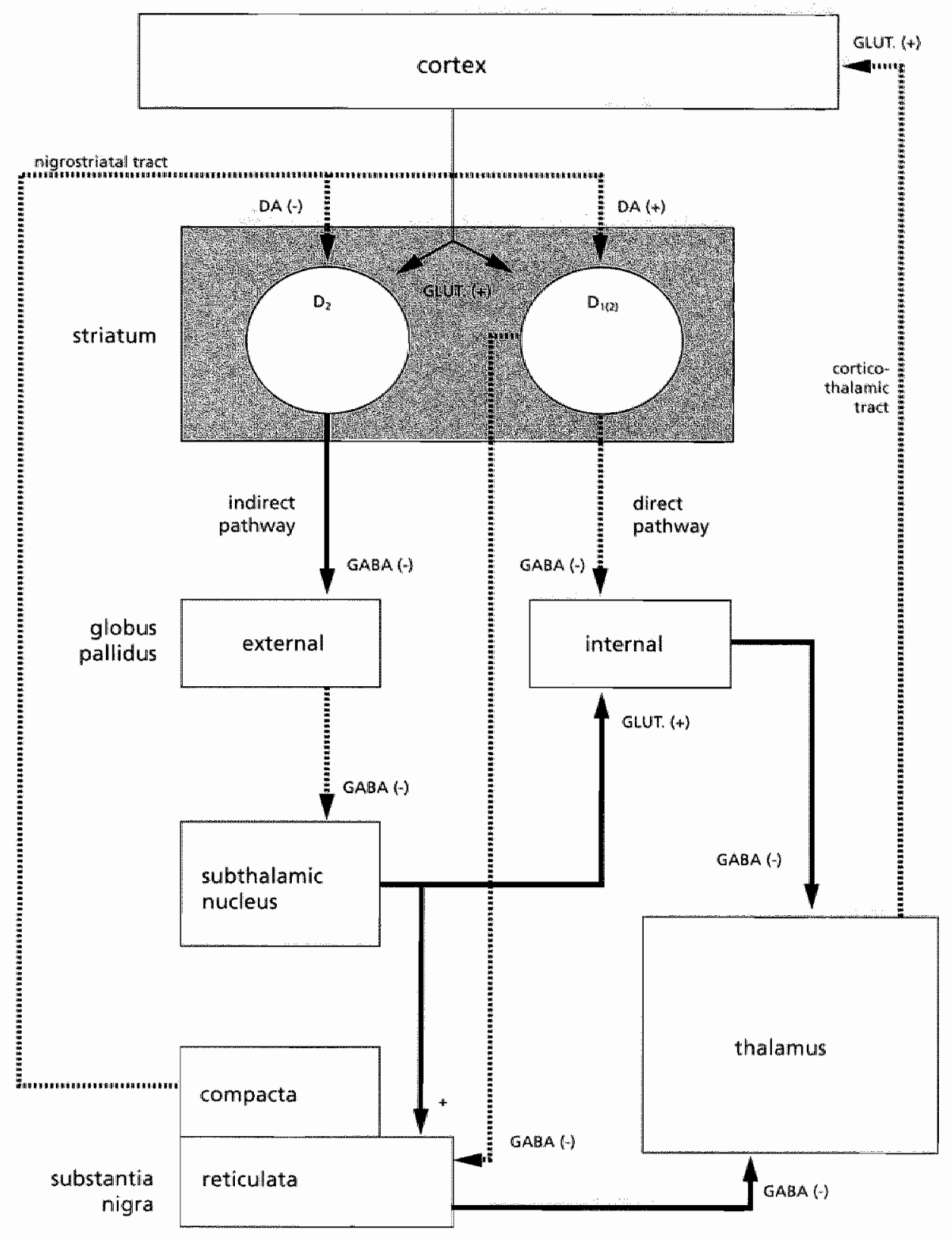


of acute tryptophan depletion (ATD). This method provides an elegant tool to acutely reduce the serotonin levels in the brain by means of an amino acid mixture which lacks the precursor of serotonin, tryptophan. The chapter will mainly focus on the effects of ATD on aspects of motor function and cognition in PD patients.

Chapter 6 will discus the effects of ATD on mood and cortisol in patients suffering from $\mathrm{PD}$. This chapter will test the serotonergic hypothesis for depression in PD, which states that a reduced serotonergic tone can be seen as a physiological compensation mechanism for the reduced dopamine activity in PD patients but at the same time is a risk factor for depression.

The contents of chapter 7 will deal with another, and opposite technique used for experimentally influencing the serotonergic system: acutely challenging serotonin instead of acutely depleting it. Challenging the serotonergic system in humans was realized by means of ingestion of a selective serotonin reuptake inhibitor (SSRI), or a 5-HTla antagonist. Both techniques have the same end effect: an acute, temporarilly, and fully reversible rise of serotonergic levels The outline is comparable with chapter 4 , in that the test battery used for both experiments is identical.

Chapter 8 describes a new task, developed in collaboration with the department of Movement Sciences of Maastricht University. The Manual Tracking Task for Tremor Assessment (MTT-TA) was developed in order to investigate problems in the execution of simple movements in PD patients. This task was performed in both the ATD experiment and the challenge experiment. Data from both experiments were combined in this chapter.

In Chapter 9 the main findings are summarized and discussed. Clinical implications and directions for future research are given.

\section{References}

111 Parkinson I, Am assay on the Shaking Palsy, London: Sherwood, Neely and Jones, 1817.

21 Hornykiewica 0, Die topische Lokalisation und das Verhalten von Noradrenalin und Dopamin (3-Hydroxytyramin in der Substankia Nigra des nomalen und Parkinsonkranken Menschen, Wien Klin Wschr. 196375309 . 312

[3] Geffen CR. Dopamine receptor function in the basal ganglia. Cin Neuropharm, 1995 18 5162.5177.

14] Vam Praag HM and De Haan S, Central serotonin metabolism and frequency of depression. Psychiatry Res, 1979 $1219-224$

15] Cunmings IL, Depression and Parkinson's disease; a review, Am J Psychiatry, 1992 149 (4) 443-454. 
(6) Leentiens Af: Depression in Parkinson's oitease: conceptual issues and dinical challenget, I Geriatr Psychiatry Neurol, 2004.17 (3) 120-125.

(7) Birkmayer W and Birkmayer J., Dopamine action and disorders of neurotransmitter balance, Gerontology. 1987 $33(3-4) 168-171$.

(8) Shannak K, Rajput A, Rozdisky B, Kish S, Gibert J and Homykiewicz O, Noradrenaline, dopamine and serotonin levels and metabolism in the human hypothalamus: obserwations in Pakinson's disease and normal subjects. Brain Res, 1994639 (1) 33-41.

(9) Jellinger $K$, Overview of morphological changes in Parkinson's disease, Adv Neurol, 198645 1.18.

110] Johansson B and Roos BE, 5-tydroxyindateacetic and homovanillic acid levels in the cerebrospinal fluid of healthy volunteers and patients with Parkinson's syndrome, Lffe Sci, 19676 (13) 1449-1454.

[11] Kostic VS, Diuricic BM, Covickowic-Sternic N, Bumbasirevic $L_{2}$ Nikolic M and Mrsulja BB, Depression and Parkinson's disease: possible role of serotonergic mechanismus, J Neurol, 1987 234 (2) 94-96.

(12) Mayeux R, Steen Y, Cote L and Wiliams IB. Altered serotonin metabotism in depressed patients with parkinson's disease, Neurology, $198434(5) 642 \cdot 646$

113] Mayeux R, Stern Y, Williams JB, Cote L. Frantz A and Dyrenfurth I, Clinical and biochemical features of depres. sion in Parkinson's disease, 1986 143:6) $756-759$.

14] Toghi $H$, Abe T, Saheki M, Yamazaki $K$ and Murata I, Concentration of catechollamines and indolearmines in the cerebrspinal fluid of patients with vascular parkinsonism compared to Parkinson's disease patients, J Neur Trans, $1997104441-449$.

115] Jacobs BL and Fornal CA, 5-HT and motor control: a hypothesis, Trends Neurosd, 199316 (9) $346-352$.

(16) Riedel WJ, Klaassen T and Schmitt JA, Tryptophan, mood, and cognitive function, Brain Behav Immun, 200216 (5) 581.589 .

(17) Riedel WJ, Cognitive changes after acute tryptophan depletion: what can they tell us?, Psychol Med, 200434 (1) $3-8$.

[18] Riedel WI, Klasassen T, Deutz NE, van Someren A and van Praag HM, Tryptophan depletion in normal volunteers produces selective impairment in memory consolidation. Psychopharmacology (Berl). 1999141 (4) 362 .369.

[19| Schmitt JA, Jarissen BL, Sobczak S, van Boxtel MP, Hogervorst E, Deutz NE and Riedell WI, Tryptophan cepletion impairs memory consolidation but improves focussed attention in healthy young volunteers, I Psychopharnacol. $200014(1) 21129$ 
CHAPTER 2

\section{Serotonergic mechanisms in Parkinson's Disease}

Opposing results from predinical and clinical data

B. Scholtissen ${ }^{a}$, F.R.J. Verhey ${ }^{a}$, H.W.M. Steinbusch ${ }^{\circ}$, A.F.G. Leentjens ${ }^{a}$

Department of Psychiaty and Neuropskchology. Institute of Brain and Behaviour, Matstricht Unikersity.

Mastricht, The Netheriands

Journal of Neural Transmission (accepted)

\section{Abstract}

Parkinson's Disease (PD) is a neuropsychiatric disease affecting approximately 75-175 per 100.000 people in the general population. The classical triad of symptoms, tremor, rigidity, and akinesia is mainly caused by degeneration of dopaminergic neurons from the substantia nigra. However, other neurotransmitter systems also show signs of degeneration, among which the serotonergic system. The exact role of serotonin in PD remains unclear. We present here a review about functional serotonergic interventions and serotonergic imaging studies in PD, and will go into the importance of combining preclinical and clinical research data in order to gain more insight into the role of serotonin in PD. More specifically, the present review is aimed at bridging the gap between data from animal models of PD and data from human research.

\section{Introduction}

Parkinson's Disease (PD) is a neuropsychiatric disease affecting approximately $1 \%$ of the population over the age of 50 years [11. It's most prominent symptoms are tremor, rigidity, and bradykinesia. However, nonmotor symptoms such as cognitive and affective symptoms are also commonly observed. The principal pathological characteristic of $P D$ is the degeneration of dopamine (DA) producing neurons in the substantia migra 
pars compacta (SNC), which accounts for most of the motor systems. Relatively little research has been focused on other parts of the dopaminergic system and on the influence of other neurotransmitter systems. The fact that other neurotransmitter systems, e.g. the noradrenergic, chollinergic, and serotonergic system, are also involved in PD, has since long been known [2,3]. Postmortem research has shown reduced levels of basal ganglia 5-HT, and in vivo studies have shown decreased levels of the 5-HT metabolite 5-hydroxy-indoleatic acid (5HIAA) [4-61. Reduced serotonin levels are often associated with a risk for depression (7). Depression is one of the most common comorbid disorders observed in PD patients. The incidence of depression in PD ranges from 2-70\%, depending on the population tested, and the methods used for testing. Generally, a mean percentage of $25-40 \%$ is reported in literature 18,91 . Furthermore, serotonin is known to influence cognitive symptoms, notably memory consolidation $\| 10$. It may also be involved in the regulation of motor processes. In animal research it is generally stated that serotonin has an inhibiting effect on striatal dopamine release [11-13], although some groups report an agonistic interaction between serotonin and dopamine $\left[14_{n} 15\right]$. Moreover, 5-HT receptor subtypes differentilally influence DA release [16]. The 'serotonergic hypothesils for depression in PD' is based on the presumption of an antagonistic interaction (17). This hypothesis states that the reduction of serotonin in PD patients is a compensatory mechanism for the reduced dopamine level in the striatum, at the cost of an increased risk for depression.

This paper will review (recent) literature about functional serotonergic interventions and serotonergic imaging studies in PD, and will give an overview of the findings concerning this relation and the importance of combining preclinical and clinical research data in order to gain more insight into the role of serotonin in PD. More specifically, the present review is aimed at bridging the gap between data from animal models of PD and data from human research.

\section{Methods}

This review consists of two parts: the first part will explore the preclinical field of research, the second part will focus on the clinical field. Studies for this review were identified by searches of MEDLINE and references from relevant articles. The following search terms were used: "Parkinson's disease", "serotonin", "serotonin and PD", "serotonin and Parkinson's disease", "functional interventions and serotonin and Parkinson's disease", "serotonin and Parkinson's disease models", "rodent Parkinson's disease models and serotonin", "primate Parkinson"s disease models and serotonin", "serotonergic autoradiography and Parkinson's disease", "serotonin positron emission tomography imaging and Parkinson"s disease", and "serotonergic imaging and Parkinson"s disease". 
Appropriate articles on both animal models of PD and human PD were selected. Conference proceedings and relevant PhD theses were also included.

\section{Parkinson's disease models in animals}

\section{Modeling PD in animals}

PD is a human disease and does not naturally occur in any other species. In order to gain insight into the possible pathological mechanisms of $P D$, valid experimental models have to be created. There are several different ways of modeling PD. The most widely used modiels make use of the toxins 1-methyl-4-phenyl-1,2,3,6-tetrahydropyridine (MPTP) (mainly used in mice and primates) and 6-hydroxydopamine (6-OHDA) (mainly used in rats), but recent models have also used proteasome inhibitors to model PD in animals $[18,19]$. The MPTP model of PD is based upon exposure to the neurotoxin MPTP.

In 1983 it was reported by Langston and colleagues that a number of young drug users developed a rapidly progressive parkinsonian syndrome after using 1-methy|-4-phenyl-4-propion-oxypiperidine (MPPP), an analog of the narcotic analgesic meperidine. However, the drug users used a contaminated version of MPPP, and MPTP was the responsible neurotoxic contaminant [20]. These patients responded to standard anti-parkinsonian levodopa treatment. The similarity between these patients and patients suffering from PD led to the development of animall models using MPTP as a PD model.

Besides primates, rodents (rats and mice) are widely used species for investigating various disease processes. The introduction of the catecholaminergic neurotoxin 6-OHDA provided a relatively easy way of destroying noradrenergic and dopaminergic neurons in these animals 121$]$. Since the degeneration of the dopaminergic nigrostriatal pathway is the major cause of the motor symptoms observed in $\mathrm{PD}$, lesioning this pathway (by means of locally injecting 6-OHDA) provided a model of PD. Injecting animals with desimipramine, a noradrenalin transporter blocker which inhibits the uptake of 6-OHDA into the noradrenergic neurons, 6-OHDA will mainly destroy the dopaminergic fibers and leave the noradrenergic fibers relativelly unharmed.

Several 6-OHDA models of PD exist and can be divided into three categories. 6OHDA was either injected into the Caudate Putamen complex (CPU, or striatum), the Medial Forebrain Bundle (MFB) or the Substantia Nigra pars compacta (SNC) itself. For a review see Deumens et al. and Orth et al $\{22,23 \mid$. Dopamine producing neurons are mainly situated in the SNC and project to the CPU via the MFB, so lesioning either one of these areas with 6-OHDA results in a decrease of dopaminergic levels, similar to the human situation. There is, however, no general agreement on which model is the best 
for modeling PD. Regarding the clinical situation, a partial bilateral model would resemble human PD best, but bilateral 6-OHDA models are not the most widely used.

\section{Serotonin in animal models of Parkinson's disease}

As mentioned before there are a number of different 6-OHDA models by which certain aspects of $\mathrm{PD}$ (e.g. slowing of reaction and motor times) can be modeled. 6-OHDA mimics the dopaminergic degeneration seen in PD in humans, but the validity of this model for human PD can be questioned, since data from neuropathological and serum samples from suicidal patients shows that dopamine is not the only degenerating neurotransmitter system. Serotonergic projections originating from the dorsal raphe nuclei innervate all parts of the basal ganglia circuitry [24]. It might therefore be plausible that 5-HT plays a role in the regulation of movements executed by the basal ganglia. In the human situation it has been shown in both in vivo and post mortem research that 5-HT transmission in the basal ganglia is indeed altered $[6,25]$. There are several ways of investigating the serotonergic system. This part of the review will focus on (acute) serotonergic interventions and imaging studies in animal models of PD.

\section{Serotonin dopamine interaction}

The available preclinical literature regarding interactions between $5-H T$ and DA reports opposite findings. On the one hand there are groups stating that 5-HT exerts an inhibitory effect on striatal DA $|11-13|$. On the other hand there are groups that have presented data stating that $5-\mathrm{HT}$ actually facilitates DA outflow [14,15]. More recent research has shown that at receptor subtype level a distinction can be made between receptor subtypes which either constitute an excitatory $\left(5-\mathrm{HT}_{2 \mathrm{a} / 2 \mathrm{~b}}\right)$ or inhibitory $\left(5-\mathrm{HT}_{2 \mathrm{c}}\right)$ influence on DA release in the striatum, and furthermore, a distinction can be made between influence which is exerted either during tonic or phasic activity of DA neurons $112,16,26 \cdot 31]$. These observations have to be taken into account when interpreting preclinical data, and when extrapolating animal data to the clinical situation.

\section{Serotonergic intervention}

The serotonergic transmitter pathway can be subdivided into a number of different pre- or postsynaptic receptor subtypes distributed throughout the brain and peripherall nervous system, of which the most important for $\mathrm{PD}$ and motor processes are the 5$H T_{i a}, 5-H T_{i b}, 5 \cdot H T_{2 a f c}$ receptor subtypes [32].

$5-\mathrm{HT}_{1 a}$ receptors are widely distributed throughout the basal ganglia. They are located on dorsal raphe neurons with efferents to the striatum, and are also localized on cortical neurons sending glutamatergic projections to the basal ganglia [33]. In an experiment by Gerber and colleagues it was found that $5-\mathrm{HT}_{1 \mathrm{a}}$ receptor stimulation represented antiparkinsonian effects in 6-OHDA lesioned rats [34|. This effect is most like- 
Iy caused by the increase in 5-HT, receptor activation, resulting in an inhibition of 5 . $H T$ release. However, research by De Deurwaerdere and colleagues and Lucas and col. leagues reported results speaking against the abovementioned observations $\mid 13,35]$.

In 1995, Numan and colleagues showed that unilateral 6-OHDA lesioning of the nigrostriatal pathway resulted in a significant increase in the hybridization density for $5-\mathrm{HT}_{2}$ receptor mRNA in the CPu ipsilateral to the lesion side [36]. In 1996, Laprade and colleagues made similar observations [37]. This finding indicates a relationship between striatal dopaminergic and serotonergic mechanisms. Similar findings were observed earlier in both rodents and primates $[38,39]$. In addition, in a study by Maeda and colleagues, it was reported that extensive DAergic denervation in adult rats induced rapid serotonergic hyperinnervation in the striatum as early as 2 weeks after lesioning 1401. Similar findings were also reported by Mignon and colleagues, although they did not use a PD specific model, but used reserpine instead to induce a mono-amine depletion [41]. Fox and Brotchie found that intracerebral infusion of a selective $5-\mathrm{HT}_{2 \mathrm{C}}$ receptor antagonist into the substantia nigra pars reticulata had an antiparkinsonian action in 6-OHDA lesioned rats. These antagonists exert a potentiating effect on the $\mathrm{D}_{2}$ agonist quinpirole resulting in a reduction of $P D$ symptoms [42]. They also observed a beneficial effect of $5-\mathrm{HT}_{2 c}$ receptor antagonists given in combination with dopamine $\mathrm{D}_{1}$ receptor agonists. This indicates that the combination of these kinds of drugs might give a better result than just $D_{1}$ agonists alone [43]. The effects of quetiapine a $5-H T_{2 a / c}$ and $D_{2 / 3}$ receptor antagonist showed similar results in both 6-OHDA lesioned rats and MPTP lesioned primates [44]. Bishop and Walker found that concurrent intrastriatal stimulation of D1 and 5 -HT2 receptors within the dopamine depleted striatum induces synergistic locomotor behavior [45]. A study by our own group, investigating the effects of the 5 $\mathrm{HT}_{2}$ receptor agonist DOI and DA agonist d-amphetamine in a bilateral 6-OHDA model, showed that serotonin and dopamine appeared to be agonistically linked to each other (Scholissen et al. submitted).

Another substance is 3,4-methyldioxymethamphetamine (MDMA), or ecstasy. This substance also exerts some serotonergic effects, but until now it remains unclear what the precise mechanism of action of MDMA is. A few preclinical studies report the ef fects of this substance. One study by Iravani and colleagues on the effects of MDMA on MPTP treated primates (common marmosets) showed that MDMA was able to reverse L-dopa induced dyskinesia and motor activity in L-dopa primed common marmosets, while the inhibition of the serotonin transporter by fluvoxamine completely inhibited the effects of MDMA on locomotor activity [46]. These results suggest that the effects of MDMA might heawily rely on serotonergic mechanisms. In another study by Lebsanft and colleagues, the effects of MDMA derivates were tested in a unilateral 6 . OHDA model. They found that the effect of MDMA and its derivates on rotational behavior was, at least partially, mediated via the serotonergic neurotransmitter system, 
but there also is evidence that MDMA influences motor behavior through other neurotransmitters like for instance norepinephrine and dopamine 147$]$. The effects are thus not selective enough for MDMA to be considered a potential candidate for serotonergic alleviation of parkinsonian symptoms.

\section{Neuro-imaging}

Another way of investiglating the function of a particular neurotransmitter is by means of imaging. This method is widely used in the human situation for investigation of serotonergic functioning in PD (see below), but in animal models of PD imaging methods have thus far not been used for this purpose.

The only method that has been used for investigating serotonin in a PD model is autoradiography, which actually is no imaging method. There are two studies reporting on 5-HT autoradiography in a PD animal model. In a study by Frechilla and colleagues, 5-HT1a receptor expression was enhanced in MPTP treated primates [48]. The hypothesized up regulation of this receptor subtype might represent a compensatory mechanism for the dopaminergic dysfunction in neurodegenerative diseases such as PD. In another autoradiography study it was found that 6-OHDA lesioning of the medial forebrain bundle led to hyperinnervation of 5-HT nerve terminals and increases in basal extracellular 5-HT levels [49].

\section{Human Parkinson's disease}

The second part of this review will focus on functional and imaging studies performed with respect to the serotonergic system in patients with $\mathrm{PD}$. As mentioned earlier, the dopaminergic neurotransmitter system is not the only system that is affected in PD. Postmortem neurochemical studies have found reduced noradrenergic, cholinergic and serotonergic activity, and concerning serotonin, reductions of up to $50 \%$ of serotonin have been found in areas of the cortex and the basal ganglia of PD patients $[2,50,51]$. But there are a number of drawbacks to postmortem studies, therefore, in vivo studles and especially functional studies (be it direct interventions of the serotonergic system or functional imaging studies) might provide a better insight in to the role of serotonin in $\mathrm{PD}$.

Two main methods of investigating the serotonergic system can be distinguished. The easiest, and most direct, way to intervene with this system can be achieved by means of decreasing or increasing the available amount of serotonin and thereby acutely altering serotonergic activity (be it decreasing or increasing serotonin content). Another way of investigating the serotonergic system is by means of functional neuroimaging, this will be discussed later. 


\section{Acute serotonergic intervention}

\section{Acute Tryptophan depletion}

For the production of serotonin the precursor L-tryptophan is needed, which the human body does not produce autonomously. This means that the precursor has to be obtained from food intake. An increased dietary intake of tryptophan, results in an increase in the synthesis of serotonin [52]. Conversely, when tryptophan is left out of the diet, serotonin synthesis will drop rapidly after only a few hours.

Acute tryptophan depletion (ATD) is a widely used method to create an acute decrease of serotonin in the brain. The method is based on the intake of a mixture (after an overnight fast) containing a balanced amount of large neutral amino acids (LNAA's). waline, leucine, isoleucine, phenylalanine, and tyrosine, but no tryptophan. The reduction of tryptophan availability is achieved by means of two mechanisms. First, the synthesis of proteins, which requires tryptophan and, therefore, reduces peripheral tryptophan levels is stimulated. Second, the LNAA's all use the same transport mechanism for passage over the blood brain barrier. This results in a competition among the LNAA's (thus also tryptophan) at the blood brain barrier. Because there is no tryptophan in the mixture, the other LNAA's will enter the brain in llarger numbers than the available tryptophan, resulting in a steep decrease of serotonin production. The tryptophan levels reach a minimum after approximately 5 to 7 hours, and levels will return to normal upon return to a normal diet $[53 \mid$.

ATD has been widely used in research in both normal and psychiatric popullations to study its effects on several aspects of cognition and mood. Lowering of mood in patients with a mood disorder in remission, their first degree relatives, and first degree relatives of patients with bipolar disorder is reported [54-57]. ATD also exacerbates anxlety, panic and aggression in vullnerable individuals $158-601$. Last, ATD also influences learning, memory and executive functions and focused attention $110,59,61,621$.

Concerning PD, however, ATD is only mentioned in one case history. In the study of McCance-Katz et al. [63), a 55 year old man with PD underwent ATD four weeks after remission of a major depression which was treated with fluvoxamine. During ATD, the Hamilton Rating Scale for Depression (HAMD) increased from 7 to 18 ( 13 being the cutoff for minor depression and 21 for major depression), and a visual analog scale (VAS) for depression (a subject-rated measure of mood state on a $0-100 \mathrm{~mm}$ scalle: $0=$ no depression, $50=$ moderate depression, $100=$ extreme depression) increased from 10 to 50. Not only did the mood of the patient change under influence of ATD, but also the motor symptoms (as measured by the Unified Parkinson's Disease Rating Scale (UP. DRS) increased from 19 to 34 points. During the placebo condition, where the patient consumes a mixture containing tryptophan and the other LNAA's in a balanced combination mood did not change. The score of the UPDRS, however, did again increase 
from 27 to 39 points. The mood lowering effect in the active condition was expected, but the worsening of motor function was not. The last was attributed to the reduction of central L-dopa concentrations after the amino acid load. Our group has performed the first experimental investigation of the effects of ATD in a group of non demented, non depressed PD patients and results showed that the effects of ATD on mood, cognition, and motor performance, were similar in both PD patients and healthy controls. Results indicated that there were no effects on mood, however, ATD had a detrimental effect on aspects of memory function, and beneficial effects on aspects of motor function (scholtissen et al. I Neurol So (accepted); Leentijens et al., Neuropsyphar (acceptedy). There have been no further studies since, inwestigating the possible effects of ATD on mood or motor symptoms of PD.

\section{Aspecific serotonergic antagonism}

The first study investigating the effects of serotonim antagonism on PD was the study by Klawans and colleagues 164|. In the study of Klawans and colleagues [64], the effect of treatment of PD patients with methysergide, a serotonin antagonist, was investigated. No improvement of motor function was observed. Motor function was measured with the Northwestern Disability Scale [65]. This, however, is not a very sensitive scale, and therefore any subtle changes might have been overlooked. There were a number of other drawbacks to this study. It was an open study, and methysergide is a partial antagonist, also exhibiting partial $5-H \mathrm{~T}_{1 a}$ agonism.

In a study by Avila and colleagues $\| 66$, the effects of nefazodone, a serotonin reuptake inhibitor and postsynaptic 5-HT2a antagonist, on both depressive and motor symptoms was investigated in a single blind experiment. Results showed that nefazodone was as effective as fluoxetine regarding the treatment of depressive symptoms, but that the effects of nefazodone on the motor symptoms were significantly better than those of fluoxetine. This beneficial effect was ascribed to the selective $5-\mathrm{HT}_{2}$ antagonistic effects of nefazodone. According to Avila and colleagues, blocking 5 - HT 2 receptors promotes dopamine release and a subsequent reduction of $\mathrm{D}_{2}$ receptor blockade. resulting in a reduction of extrapyramidal symptoms.

In an open label study by Zoldan and colleagues $|67|$, the effects of odansetron, a 5 $\mathrm{HT}_{3}$ receptor antagonist, were tested in advanced $\mathrm{PD}$ patients suffering from psychosis. They observed an improvement of psychotic symptoms, without worsening motor function, which may indicate that antagonizing $5-\mathrm{HT}_{3}$ does not influence DAergic release.

\section{Aspecific serotonergic loading}

There ale a number of different methods for increasing the overall serotonergic activity in the brain. Some of these use serotonin precursors (L-tryptophan, and 5-hydroxytryptofan (5-HTP)) as a challenge method, while others used aspecific serotonergic agonists. 
like fenfluramine or selective serotonin reuptake inhibitors (SSRI's). Not all of these methods have been used for studying acute changes in symptomatology or neurochemical parameters. A number of these methods were considered as possible treatment methods and hence the effects of long term administration were studied. Because of the useful information of these treatment studies, they will also be discussed here.

Before the proposition of the 'serotonin' hypothesis by Mayeux, research focused on restoring the depleted levels of serotonin in patients with PD by prescribing them serotonin precursors. In the study of Coppen et al. improvement of tremor, rigidity, akinesia and gait was reported after the addition of 900 to $3000 \mathrm{mg}$ tryptophan to levodopa therapy in 40 patients for at least 8 weeks [68]. A study of Sandyk and Fisher described amelioration of levodopa induced 'on-off' fluctuations in 2 female patients after tryptophan administration [69|. Other research found an amelioration of levodopa induced visual hallucinations or mental deterioration, without negative effects on extrapyramidal symptoms (70-72].

Hall and colleagues described no effect on motor function after tryptophan administration in 3 PD patients, and worsening of motor function after additional prescription of pyridoxine (vitamin $B_{6}$ ), whereas pyridoxine alone did not have any effect [73]. Pyridoxine can be given in combination with decarboxylase, this increases the effectiveness of decarboxylase. Beasly, however, described that treatment with 2 to 6 grams of tryptophan in a double-blind and placebo-controlled design did not have any influence on both the psychiatric and motor symptoms of 9 PD patients 1741. Bryant commented that a subgroup of older patients with a relative recent disease onset may benefit from L-tryptophan, whereas patients with a longer disease duration might experience adverse effects [75]. This is interesting because in patients with a relative short disease duration the degeneration of several neurotransmitter systems (among which serotonin) will not be as severe as in patients with a longer disease duration. From this it might be concluded that extra intake of tryptophan might only be effective in relatively early stages of the disease, when degeneration is still mild, and may even be disadvantageous at later stages of the disease. To make it even more puzzling, a study by Chase and colleagues described a worsening of extrapyramidal symptoms, except tremor, in 7 patients treated with 5-HTP [76].

With respect to treatment purposes the interest in serotonin precursors has faded, and interest focused almost completely on the dopaminergic neurotransmitter system. For research, better methods have become available to increase serotonin levels without directly interfering with the serotonin synthesis. Fenfluramine, a serotonin releasing agent, has long been studied as a potential serotonergic treatment option in PD. Beasly and colleagues found that fenfluramine hydrochloride had no influence on extrapyramidal symptoms in 5 medication free and 5 levodlopa treated PD patients [74]. From animal studies it is known that fenfluramine also inhibits tryptophan hydroxylase, 
the rate limiting enzyme in the synthesis of tryptophan, and may in fact lower central serotonin activity $[77]$.

As a challenge paradigm, the prolactin and cortisol response to fenfluramine are seen as an indicator of central serotonergic function. Blunted prollactin response to fenfluramine challenge has been described in mood disorders and in disorders of impulse control [78]. Both enhanced and blunted responses have been reported in panic disorder. Results from different studies are not consistent, and cortisol responses have been even less consistent. An overview studies in different psychiatric disorders is given by Newman [79].

In PD, two studies have used the fenfluramine challenge test (FCT) in order to assess serotonergic function. Effects of a single dose of $60 \mathrm{mg} \mathrm{D}$-fenfluramine on prolactin and cortisol were investigated by Kostic and colleagues in a population of 22 PD patients, 11 depressed PD patients and 20 healthy controls. Prolactin response was significantly blunted in PD patients, compared to controls. The response of depressed PD patients was significantly more blunted than that of non depressed PD patients. Cortisol responses did not differ between the groups $180 \mathrm{j}$. Volpi et al. investigated adrenocorticotrope hormone (ACTH) and cortisol response to D-fenfluramine in 10 PD patients and 10 controls. Both the ACTH and cortisol responses were blunted in PD patients 181$]$. These studies suggest a defective serotonergic control of the hypothalamic-pituitaryadrenal (HPA) axis in PD patients, and can be seen as evidence for a more generalized dysfunction of the central serotonergic system.

\section{Receptor specific challenge}

Challenging the serotonergic system by means of an SSRI might reveal some informaltion about its role in PD, but is too aspecific to provide exact information about specific actions of receptor subtypes. Therefore, a receptor specific challenge will provide more information on the exact mechanism of different receptor subtypes in PD. A number of studies have been performed, especially regarding the $5-\mathrm{H} T_{1}$ and $5-\mathrm{HT}_{2}$ receptor subtype. In a study by Hildebrand and colleagues, an improvement of resting tremor in PD patients was observed after treatment with ritanserin, a 5-HT2 receptor antagonist [82]. In a singlemblind placebo controlled study, Henderson and colleagues reported an improvement of akinesia and gait, but not of tremor, after administration of ritanserin [831. Volpi and colleagues demonstrated a blunted growth hormone response in PD patients after stimulation with sumatriptan, a $5-\mathrm{HT}_{1}$ receptor agonist 1841 . Their conclusion was that these results indicate an impairment of 5-HT, receptor mediated serotonergic transmission in the control of growth hormone secretion, suggesting that this specific deficit might alter other serotonin mediated mechanisms in the PD brain.

The study by Sempere and colleagues. [85] investigated the effects of the prokinetic agent cisapride. They observed a worsening of parkinsonian tremor in two patients with parkinsonism. They hypothesized that the effect of cisapride was partially caused 
by an agonistic action on the $5-H T_{4}$ receptor subtype. Since this is the only study addressing the $5-\mathrm{HT}_{4}$ receptor subtype and only two patients were described in this study, the results should be interpreted with caution.

\section{Neuro-imaging}

Another way of investigating neurotransmitter activity is by imaging. A number of methods are used: Single Photon Emission Computed Tomography (SPECT), Positron Emission Tomography (PET), and functional Magnetic Resonance Imaging (FMRI). These techniques are mostly used for the diagnosis of PD but also provide information about neurochemistry, metabolism and general brain activity, and might therefore also lead to a better understanding of the pathophysiological serotonergic mechanisms in PD.

\section{SPECT imaging}

The main focus of SPECT studies has been on the serotonin transporter (5-hydroxytryptamine transporter, 5-HTT). 5-HTT can be measured by means of the SPECT ligand 1231B-carboxy-iodophenyl-tropane ( $B-C I T)$. This ligand binds to both the dopamine transporter (DT) and 5-HTT, but at different time points after tracer injection (20-30 hours after injection for DT and 2-4 hours after injection for 5-HTT) $[86,87]$. Two studies reported no a relation between the 5-HTT binding in the hypothalamic and midbrain region with motor, mood or cognitive symptoms, but they based their statements on data obtained 20-24 hours after injection of the B-CIT ligand when binding to DT transport= er is reaching a maximum, and $5-\mathrm{HTT}$ binding is long past its optimum $[88,89]$.

Haapiniemi and colleagues investigated both DA and 5-HTT levels in drug naive PD patients by performing two scans (four and twenty hours after tracer injection) in each subject. They demonstrated reduced 5-HTT activity in the thalamus and medial frontal areas $|90|$. Kim and colleagues investigated DA and 5-HTT activity in a group of fortyfive PD patients in 2003 and this time performed two scans for DA and 5-HTT activity respectively. Their results suggest that $5-\mathrm{HTT}$ levels in the midbrain region may not be affected in relatively early stages of PD. They also state that it might be plausible that 5-HTT in the remaining neurons may be up regulated, thereby raising the midbrain 5HTT density to almost normal levels [91].

In the first in vivo study investigating the $5-H T_{2 a}$ receptor in $\mathrm{PD}$, our group demonstrated reduced striatal $5-\mathrm{HT}_{2 \mathrm{a}}$ receptor density in $\mathrm{PD}$ patients, as compared to matched controls, using 1231-5-R91150: a 5- $\mathrm{HT}_{2 \mathrm{a}}$ receptor specific ligand |92-95|. Reduced binding was found predominantly contralateral to the site of onset of the symptoms, and bilaterally in the area of the premotor cortex and supplementary motor area. These findings are in favor of the serotonergic hypothesis stating that down-regulation of serotonin is a compensatory response to the degeneration of dopaminergic neurons [96]. 
This process is thought to result in a decrease of motor symptoms, but on the other hand pose a risk factor for the development of a depression. Overall, SPECT imaging is a valid method for gaining more information about central serotonergic activity and distribution in PD patients.

\section{PET imaging}

Besides SPECT, PET can also be used for imaging the serotonergic system in vivo. To date, there are however, not many studies which have used this technique to inwestigate the impact of the serotonergic neurotransmitter system on PD. In a study by Doder and colleagues, a reduction of $27 \%$ in $5-\mathrm{HT}$ ia binding was observed in the midbrain raphe using "C-WAY 100635 as a ligand [97]. Kerenyi and colleagues observed that a decrease in distribution volumes of "C(+) MCN5652 (a 5-HT ligland binding to the serotonin transporter) in the caudate nucleus and the putamen was related to disease stage [98]. A drawback of using ${ }^{11} \mathrm{C}(+) \mathrm{MC} N 5652$, is that this ligand demonstrates considerable nonspecific bind ing.

To date, no other studies have investigated the serotonergic system in PD patients by means of PET imaging.

\section{Discussion}

From the existing literature, reporting on both in vivo and postmortem data in animal models and in humans, it is apparent that the serotonergic neurotransmitter system is involved in the pathophysiology of PD. In animal models of PD serotonergic research has mainly focused on the $5-\mathrm{H} T_{1}$ and $5-\mathrm{HT}_{2}$ receptor subtype. Results from these studies indicate that stimulating $5 . \mathrm{HT}_{1 / \mathrm{a}}$ receptors and inhibiting $5-\mathrm{HT}_{2}$ receptors resulted in an antiparkinsonian effect. More specifically, 5- $\mathrm{HT}_{2 \mathrm{a} / 2 \mathrm{~b}}$ receptors exert an excitatory and $5-H T_{2 c}$ receptors exert an inhibitory influence on dopamine release [16]. However, the overall picture still is confusing. This leaves room for speculation about the exact value of preclinical research for clinical PD. Regarding the sparse results from MDMA research it can be concluded that although MDMA improves parkinsonian symptoms, the exact mechanism by which this effect is achieved remains unclear. Autoradiographic studies show that botth after MPTP and after 6-OHDA lesioning, a hyperinnervation of serotonergic fibers occurs. This might represent a compensating mechanism for the damaged dopaminergic system. Results from Tanaka and colleagues showed that with progressive degeneration of DAergic neurons in $P D_{\text {. }} \mathrm{DA}$ formation increasingly takes place in striatal serotonergic nerve terminals $[99,100)$.

In clinical studies investigating PD, acutely lowering serotonin levels by means of ATD led to a worsening of both mood and motor symptoms in one case study [63], but 
in a study by our own group no effects on mood were observed. The effects of ATD appeared to be similar in both patients and control subjects impairing aspects of cog. nition and improving aspects of motor performance (Scholvissen, ef al., J Neurol sei (accepted). Increasing serotonin levels by means of administering l-tryptophan resulted in mixed results, administration of fenfluramine either had no effect at all, or resulted in unclear effects. Selectively antagonizing the $5-\mathrm{HT}_{2 \mathrm{a}}$ receptor subtype resulted in an antiparkin. sonian effect. Taken together, challenging the serotomergic system not always resulted in comparable findings. This might imply that the existing theories of serotonergic functioning in PD have to be reviewed and possibly adjusted.

Imaging studies of PD patients showed decreased leveis of serotonin in several cortical and subcortical structures, indicating a pathophysiological involvement of serotonin in the disease process. It is, however, very likely that the degree of serotonergic degeneration depends on the stage of the disease.

In general the results from animal models appear to confirm the serotonergic hy pothesis, while in the human situation there is a lack of support for this hypothesis. There is no clear explanation for this discrepancy, although one of the explanations may be that, despite the fact that preclinical research has provided much information and knowledge about the influence of serotonim on dopamine and PD, animal models in PD may not be ideal to study human PD. There are a number of reasons for this. First, almost all preclinical models (except the Rotenone model, which has never been used for investigating the role of serotonin in PD) are relatively acute lesion models, whille the human situation is far from acute. As is known, approximately $80 \%$ of the dopaminergic fibers in the human striatum have to be lost before motor symptoms will become manifest [1011. This means that the degenerative process was already present for a number of years before the first symptoms became apparent and enough time was present for reactive adaptations to the degenerative process. This is in contrast with the precinical situation, where animals are surgically 'provided' with a comparable lesion. Furthermore, preclinical models using partial lesions show signs of recovery of function and anatomical structures over time, something which does not occur in the clinical sit. uation. Finally, not all the serotonergic receptor subtypes known in animals are comparable to the receptor subtypes known in humans, which may be evidence of a different neurophysiology of the serotonergic receptar system.

\section{Conclusion}

Predinical and clinical studies into the pathophysiology of PD yield contrary results. While animal madels seem to support the hypothesis that a reduced serotonin level is a compensation for a reduced dopamine level studies in humans do not support this 
hypothesis. Although predinical studies in animal models of PD may provide useful information on the pathophysiology of PD in humans, caution has to be taken when extrapolating predinical findings to the dinical situation. The value of animall models appears to be restricted, and therefore more attention and effort should be invested in experimental research with patients suffering from PD. Nonetheless, the current status concerning research and clinical treatment of $\mathrm{PD}$ patients would have never been possible without the research done in the predinical field. Therefore, when using an animal model of $\mathrm{PD}$ it is important to realize that different models represent different stages of the clinical situation. Taking this information in to account, animal models have proven useful for gaining basic knowledge about neurotransmitter systems and interactions between these systems. But for answering specific clinical issues, more attention should be focused on testing these questions in the best available model: the clinical situation itself, patients suffering from PD.

\section{References}

11. Adams RO and Victor H, Priciples of Neurology, New York: McGraw-Hil, 1993.

22) Scatton B, Javoy-Agid F, Rouquier L, Dubois B and Agid Y, Reduction of cortical dopamine, moradrenaline, serotonin and their metabolites in Parkinson's disease, Brain Res, 1983275 (2) 321-328.

13! Miyawalki E. Meah Y and Koller MC, Serotonin, dopamine, and motor effects in Parkinson's disease, Clin Neuropharma. col, $199720(4) 300-310$.

44) Chase TN, Serotonergic mechanisms in Parkinson's disease, Arch Neurcl, 1972 27 (4) 354-356.

[5] Chase TN, Serolonergic mechanisms and extrapyramidal function in man, Adv Neurol, $1974531-39$.

[6] Chen CP, Alder JT, Bray L, Kingsbury AE, Francis PT and Foster OJ, Post-5ynaptic 5.HTIA and 5-HT2A receptors are increased in Parkinson's disease neocortex, Ann W Y Acad Sa, 1998861288.289.

17) Van Pratag HM and De Hadn S, Central serotonin metabolism and frequency of depression. Psychiatry Res, 19791219 224.

18] Cummings JL, Depression and Parkinson's disease; a review, Am I Psychiatry, 1992149 (4) 443-454.

191 Leentiens Ar, Depression in Parkinson's disease: conceystual issues and clinical challenges, J Geriatir Psychiatry Neurol, $20041713) 120-126$

(10) Schmitt JA, Jorissen BL, Sobczak S, wan Boxtel MP, Hogerworst E, Deutz NE and Riedel WI, Iryptophan depletion impairs memory consolidation but improves focussed attention in healthy young volunteers, I Psychophamacol, 2000 1.4 (1) 21.29

[11] Jacobs BL and Fonal CA, 5-HT and motor control: a hypothesis, Trends Neurosci, 1993 16 (9) 346-352.

112. Di Matteo $\mathrm{V}_{n}$ Cacchio M, Di Giulio C and Esposito E, Role of serotanin(2C) receptors in the control of brain dopaminergic function, Pharmacol Biochem Belavi, 200271 (4) 727-734.

[13] De Deurwardere $\mathrm{P}$. Nawalles 5 , Berg KA, Clarke WP and Spampinato U, Constitutive activity of the serotonin2C receptor inhibits in vivo dopamine release in the rat striatum and nucleus accumbens, J Neurosci, 200424 (13) 3235-32.41.

[14] Benloucif 's and Galloway MP, Facilitation of dopamine release in wiwo by serotonin agonists: studies with microdialysis, Eur I Pharmacol, 1991200 (1) 1-8.

[15] Benloucif \$, Keegan M] and Galloway MP, Serotonin-facilitated dopamine release in viva: pharmacalogical characteriza. 
dion, Iharmacal Exp Ther, 1993265 (1) $373-37 \%$.

(16) Lucas $G$, De Deurwaerdere $P_{f}$ Caccia $S$ and Umberto $S$, The effect of setotonergic agents on haloneridol-induced striatat dapamine release in viva: oppasite role of $5 \times \mathrm{HT}(2 \mathrm{~A})$ and $5+4 \mathrm{~T}(2 \mathrm{C})$ receptor subtypes and significance of the haloperidol dose used, Neuropharmacology, $200039(6) 1053-1063$

[17] Mayeux $R$, Stern Y, Cote Land Willams JB, Atered serotonin metabolism in depressed patients with parkinson's disease, Neurology. 198434 (5) 642-646.

[18] Dauer W and Przedborksi S, Parkinson's Disease: Mechanisms and Models, Neuron, $200939889-909$.

[19] MCWaught KS, Perl DP, Brownell AL and Olanow CW, Systemic exposure to proteasome inhibitors causes a progressive model of Parkinson's disease, Am Neurol, 200456 (1) 149-162.

[20] Langston WW, Ballard P. Tetrud WW and irwin I, Chronic Parkinsonism in humans due to a product of meperidine-analog synthesis, Science, $1983219(4587) 979-980$.

[21] Ungerstedt U, 6-Hydroxy-dopamine induced degeneration of central monoamine neurons, Eur \& Pharmacol, 19685 (1) $107-110$.

[22] Deumens R, Blokland A and Prickaerts J, Modeling Parkinson's Disease in Rats: An Evaluation of 6-OHDA Lesions of the Nigrostriatal Pathway, Exp Neurol, 2002175 (2) 303-317.

[23] Orth M and Tabrizi SJ, Models of Parkinson's Disease, Mov Disord, 2003 18 (7) 729-737.

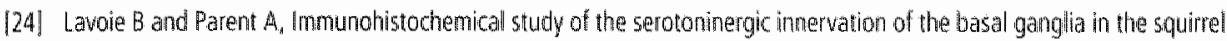
monkey, I Comp Neurol, 1990299 (1) 1-16.

[25] Hornykiewicz O, Biochemical aspects of Parkinson's disease, Neurology, 1998,51 (2 5uppl 2152-9.

[26] Di Giovanni G, De Deurwaerdere P, Di Mascio M, Di Matteo V, Esposito E and Spampinato U, Selective blockade of serotonin-2C2B receptors enhances mesalimbic and mesostriatal dopaminergic function: a combined in vivo electrophysiological and microdialysis study, Neurascience, 199991 (2) 587-597.

[27] Di Matteo W, De Blasi A, Di Giulio C and Espusito E, Role of 5-HT(2C) neceptors in the control of central dopamine function, Trends Pharmacol Sci, 200122 (5) 229.232.

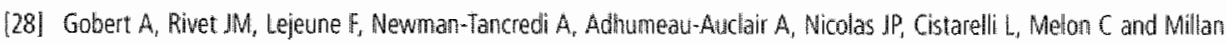
M. Serotonin(2O receptors tonically suppress the activity of mesocortical dopaminergic and adrenergic, but not serotonergic, pathways; a combined dialysis and electrophysiological analysis in the rat, Synapse, 200036 (3) 205221.

[29] Lucas $G$ and Spampinato $U$, Role of striatal serotonin2a and serotoninzc receptor subtypes in the control of in vivo dopamine outflow in the rat striatum, INeurochem, $200074693-701$.

$130 \mid$ Navailles 5, De Deurwaerdere $P$, Porras $G$ and Spanapinato $U$, in wiwo evidence that $5-H T 2 C$ receptor antagonist but not agonist modulates cocaine-induced dopamine outflow in the rat nucleus accumbens and striatum, Neuropsychopharmacology, 200429 (2) 319-326.

(31) Schmidt C, Fadayel GM, Sullivan CK and Taylor VL, 5-HT2 receptors exert a state-dependent regulation of doparninergic function: studies with MOL 100,907 and the amphetanine analogue, 3,4-methylenedioxymethamphetarinine, Eur I Pharmacol, $1992223(1) 65-74$.

[32] Nicholson SL and Brotchie JM, 5-hydroxytryptamine (5.HT, seratoniny and Parkinson's disease - opporlunilies for novel therapeutics to reduce the problems of levidopa therapy, Etur I Neurol, 200295 Suppl 31.6.

[33] Knobelmam DA, Kung HF and Lucki I. Regulation of extracelular concentrations of 5 -hydroxytryptamine (5- 417 ) in mouse striatum by 5-HT(1A) and 5-HT(1B) receptors, I Pharmacol Exp Ther, 2000292 (3) 111111117.

134] Gerber R.Altar CA and Liebman IA, Rotational behavior induced by 8-hydroxy-DPAT, a putative 5HT-1A agonist, in 6. hydroxydopamine-lesioned rats, Psychopharmacology (Bert), 198894 (2) $178-182$.

[35] Luas G, Bonhomme N, De Deurwaerdere P, Le Moal M and Spampinato U, 8-0H DPAT, a 5-HTIA agonist and ritanserin, a 5 -H $\mathrm{H}_{2 \mathrm{AC}}$ antagonist, reverse haloperidolinduced catalepsy in rats independenty of striatal dopamine release. Psychophamacology (Berl). 1997 131 (1) 57-63.

[36] Muman S, Lundgren KH, Wright OE, Herman IP and Seroogy KB, Increased expression of SHI2 receptor mPNA in rat striatum following 6- OHDA lesions of the adult nigrostriatal pathway. Brain Res Mol Brain Res, 1995 29 (2) 391-396. 


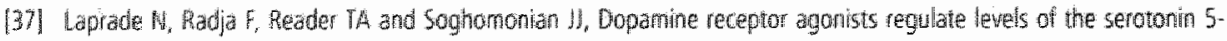
HTA receptor and its mPRA in a subpopulation of rat stiatat neurons., Neurosci, $199616(11) 3727.3736$.

[3e] Gaspar P, Febvet A and Colombo f, Serotonergic sprouting in primatte MTP-indured hemiparkinsonism, Exp Brain Res, $199396(11) 100-106$.

[39] Zhou FC, Bledsoe S and Murphy I, Serotonergic sprouting is induced by dopamine-lesion in substamtia nigra of adult vat brain, Brain Res, $1991556(1) 108-116$

[40] Maeda T, Kannarik, Shen H, Ara A, Tomiyama M, Matsunaga M and Suda T, Rapid induction of serotonergic hyperinnervation in the adult rat striatumi with extensive dopaninergic denerwation, Meurosd tett, 2003 343 (1) 17-20.

[41) Mignon $L$ and Woll WA, Posisynaptic 5-HII(A) receptors mediate an increase in locomotor activity in the monoaminedepleted rat, Psychopharnacalogy (Berl), 2002163 (1) $85-94$.

|42] Fo\% $\mathrm{SH}_{2}$ Woser $\mathrm{B}$ and Brotchie IM, Behavioral effects of $5-\mathrm{HIZC}$ receptor antagonism in the substantia nigra zona reticulata of the 6-thydroxydopamine-|esioned rat model of Parkinson's disease, Exp Neurol, 1998151 (1) 35.49

[43| Fox SH and Brotchie $1 \mathrm{M}, 5-H 72 \mathrm{C}$ ) receptor antagonists enhance the behavioural response to dopamine $0(1)$ receptor agonists in the 6-thydroxydopamine-lesioned rat, Eur I Pharmacol, 2000398 (1) $59-64$.

[44] Oh SD, Bibbiani F and Chase TH, Quetiapine attenuates levodopa-finduced motor complications in rodent and primate parkinisonian models, Exp Neurol, 2002177 (2) 557-554.

[45] Bishop $\mathrm{C}$ and Waker $\mathrm{PO}$, Combined intrastriatal dopamine d1 and serotonin 5 -hth 2 receptor stimulation reveals a mechanism for hyperlocomotion in 6-tydroxydopamine-lesioned rats, Neuroscience, $2003121(3) 649-657$.

[46] Irakani MM, Jackson MJ, Kuoppamaki M, Smith LA and Jenner P, 3,4-methylenedioxymethamphetamine (erstasy) inhibits dyskinesia expression and normalizes motor activity in 11-methyl-4-phenyl-1,2,3,6-tetrahydropyridine-treated prï* mates, INeurosi, 2003: 23 (27) 9107-9115.

[47] Lebsanft HB, Mayerhofer A, Kovar KA and Schmidt W], Is the Ecctasy-induced ipsilaterall rotation in 6-hydroxydopamine unilaterally lesioned rats clopamine independent?, J Neural Transm, $2003110(7) 707.718$.

[48] Frechilla D, Cobreros, A, Saldise L, Moratalla R, Insausti R, Luquin M and Del Rio J, Serotanin 5.HT(MA) receptor expression is selectively enhanced in the striosomal compartment of chronic parkinsonian monkeys, Synapse, $200139(4) 288$. 296.

[49] Batcioglu $\mathrm{A}$, Zhang $\mathrm{K}$ and Tarazi $\mathrm{FI}$, Dopamine depletion abollshes apomorphine:- and amphetamine-induced increases in extracellular serotonin levels in the striatum of conscious rats: a microdialysis study, Neuroscience, 2003119 (4) 10.45-1053.

150| Birkmayer Wand Bikkmayer JD, Dopamine action and disorders of neurotransmitter balance, Gerontolog\%, 498733 (3. 4. $168-171$.

[51] Birkmayer Wand Riederer $P_{2}$ Biochemische Verander ungen bei der Parkinson-Krankheit. In: Birkmayer W and Riederer $P$ (Ets.), Die Parkinson-Krankheit; Biochemie, Klinik, Therapie., Wien: Springer-Veriag, 1985, 29-59.

152! Fernstiom JD, Wurtman RI, Hamnarstrom-Waklund B, Rand WM, Munro HN and Dawidson CS, Diurnal variations in plasma concentrations of tryptophan, tryosine, and other neutral amino acids: effect of dietary protein intake, Am I Clin Nuti, 197932 (9) 1912-1922.

[53] Delgado PH. Chamey OS, Price $L H$, Landis $H$ and Heninger GR, Neuroendocrine and behavioral effects of dietary tryptophan depletion in healthy subjects., Lfe Sci, $1989452323-2332$.

[54] Deligado PL, Charney DS, Prite LH, Aghajamian GK, Landis. H and Heninger GR, Serotonin function and the mechanism of antidepressant action. Reversal of antidepressant-induced remission by rapid depletion of plasma tryptophan, Arch Gen Psychiatry, 199047 (5) 411-418.

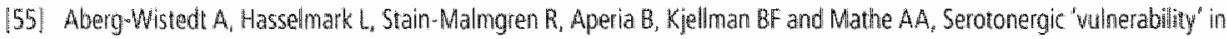
affective disorder: a study of the tryptophan depletion test and relationships between peripheral and central sesotonin indexes in citalopram-responders, Acta Psychiatr Scand, 1998.97 (5) 374-380.

[56] Klaassen $\mathrm{T}_{\mathrm{s}}$ Riedel Wi, wan Someren A, Deutz NE, Honig A and wan Praag HM, Mood effects of 24 -hour tryptophan de. pletion in healthy lirst-degree relatives of patients with affectwe disorders, Biol" Psychiatry, 199946 (4) $489-497$. 
157) Sobczak 5, Honig A, Nicolson NA and Riedel W., Eftects of acute tryptophan depletion on nood and cortisol felease in first-degree relatues of type I and type If bipolar patients and healthiy matched controls, Neuropsychopharmatology, $200227(5) 834-8.42$.

[58) Klazssen $T$, Klumperbeck 1, Deutz NE, van Praag HMN and Griez E, Efects of trypiophan depletion on anxiety and on panic provoked by carbon dioxide challenge, Psychiatry Res, 199877 (3) 167.174.

[59] LeMarquand OG, Pihl RO, Young SN, fremblay RE, Seguin JR, Pahnou RM and Benkelfat C, Tryptophan depletion, executive finctions, and disinhibition in aggressive, adolescent malles, Neuropsychophamacology, 1998 ig (4) $333-341$.

[60] Kent MM, Coplan JO, Martinez J, Kamally W, Papp LA and Gorman JM, Ventilatory effects of ryptophan depletion in panic disarder: a preliminary report. Psychiary Res, $199664(2) 83-90$.

161) Firedel WI, Klassen T, Deutz NE, van Someren A and van Praag HM, Typtophan depletion in normal volunteers produces selective imparment in memory consolidation, Psychopharmacology (Berl), 1999141 (4) 362:369.

[62] Park SB, Coull JT, MCShane RH, Young AH, Sahakian BI, Robbins TW and Cowen PI, Typtophan depletion in nomal wolunteers produces selective impaiments in learning and memory, Neuropharmacology, 199433 (3-4) $575-588$.

[63) McCance-Katz EF, Marek KM and Price LH, Serotonergic dysfunction in depression associated with Parkinson's disease, Neurology, 1992421813.1814.

[64] Klawans $\mathrm{HL}$ and Ringel SP, A clinical study of methysergide in Parkinsonism: evidence against a serotonergic mechanism, J Neurol Sci, 197319 (4) 399-405.

[65] Canter GJ, De La Torre R and Mier $M_{2}$ A method for evaluating disability in patientls with Parkinson's disease, Nerv Menit Dis, 1961 133: 143-147.

[66] Avila $A$, Cardona $X$, Martin-Baranera M, Maho P, Sastre F and Bello J, Does mefazodone improve both depression and Parkinson disease? A pillot randomized trial, J Clin Psychopharmacol, 200323 (5) 509.513.

[67] Zoldan I, Friedberg $G$, Limneh $M$ and Melamed $E$, Psychosis in advanced Parkinson's disease: treatment with ondansetron, a 5-HT3 receptor antagonist, Neurology, $199545(7)$ 1305-1308.

[68] Coppen A. Metcalle M, Carroll ID and Morris JG, Levodopa and L-tryptophan therapy in Parkinsonism, Lancet, 19721 (7752) 654-658.

[69] Sandyk $R$ and Fisher $H$, L-tryptophan supplementation in Parkinson's disease, Int J Neurosci, 198945 (3-4) 215-219.

[70] Lehmann J, Tryptophan malabsorption in lewodopa-treated parkinsonian patients. Effect of tryptophan on mental disturbances, Acta Med Scand, 1973:194 (3) 181-189.

[71] Rabey IM, Vardi I, Askenazi J] and Streifler M, L-tryptophan administration in L-dopa-induced hallucinations in elderly Parkinsonian patients, Gerontology, $197723(6) 438-444$.

172. Binkmarer W, Daniekzy W, Neumayer $E$ and Biederer $P$, The balance of biogenic amines as condition for normal behawiour, J Neural Tranism, 197233 (2) 163-178.

(73] Hall CD, Weiss EA, Morris CE and Prange AJ, Jr., Rapid deterionation in patients with parkinsonism following trypto. phan-pyridoxine addministration, Neurology. 197222 (3) 231-237.

174 ) Beasley BL, Davenpont RW and Chase TN, Fenfluramine hydrochloride treatment of parkinsonism, Arch Neurol, 1977 3.4 (4) $255-256$.

175 Bryant RC, Duration of iliness and response to tryptophan in Parkinson's disease, Arch Neural, 198037 (10) 67 .

[76] Chase TN. Ng LK and Watanabe AM, Parkinson's disease. Modilication by 5-hydroxytryptophan, Neurology, 197222 (5) $479 \cdot 484$.

[77] Fuller RW, Fenfluramine and Parkinson's disease, Arch Neurol, 197734 (111) 720.

178] Cleare AJ, Murray RM and O'Keane $V$. Assessment of serotonergic Lunction in major depression using d-fenfluamine: relation to dinical wariables and antidepressant response, Bidel Psychiatry, 1998 44 (7) $555-561$.

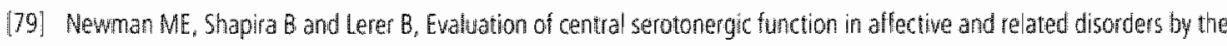
fentluramine challenge test: a critical review, Int J Neuropsychophamcol, 1998 i (1) 49-69.

180] Kostic WS, Lecic D, Dader M, Marinkovic J and Filipovic S, Prolactin and contisol responses to lenfluamine in Parkinson's disease, Biol Psychiatry. $199640(8) 769-775$. 


\title{
Functional investigations into the role of dopamine and serotonin in partial bilateral striatal 6-hydroxydopamine lesioned rats
}

\author{
Bart Scholtissena, Ronald Deumens ${ }^{a}$, Albert F.G. Leentjens ${ }^{a, b}$, Christoph Schmitz, \\ Arjan Blokland", Harry W.M. Steinbusch" "Jos Prickaerts" \\ anstitute of Brain and Behaviour. Mastricht Unwersity, Maastricht. The Netherlands \\ Department of Psychiatry, Maastricht University Hospital, Masstricht, The Netherlands.
}

Adapted from: "Functional investigations into the role of serotonin and dopamine in rats with partial bilateral striatal 6-hydroxydopamine lesions"

\section{Abstract}

Parkinson's disease (PD) is a neurodegenerative disease with degeneration of the dopaminergic (DAergic) neurons in the substantia nigra pars compacta (SNC) projecting to the caudate putamen complex (CPU) as its main cause. However, the serotonergic system also shows signs of degeneration. This led to the suggestion that alterations in the serotonergic system play a role in the pathophysiology of PD. Partial bilateral dopaminergic lesions of the CPu of rats by means of the neurotoxin 6-hydroxydopamine (6-OHDA) produce behavioral symptoms mimicking PD. In the present study the role of serotonin (5-HT) and dopamine (DA), using this model of PD, was investigated behaviorally. Reaction time (motor initiation) and movement time (motor performance) in a choice reaction time task were impaired in the lesioned animals compared to controls. The performance of rats treated with d-amphetamine or serotonergic ligands (DOI and ketanserin) in the choice reaction time task indicated that 5-HT and DA appear to be agonistically related in the CPU. The relation was the same in both control and 6-OHDA lesioned rats. Ultimately, i.e. 12 weeks after lesioning, motor initiation recovered, whereas motor performance did not. Taken together, choice reaction time responding indicated that 5-HT and DA are agonistically linked in control and 6-OHDA lesioned rats. 


\section{Introduction}

The cardinal features of Parkinson's disease (PD) are all motor symptoms, namely, resting tremor, rigidity and bradykinesia. The main cause of PD symptoms lies in the degeneration of dopaminergic (DAergic) neurons in the substantia nigra pars compacta $(S N C)$, leading to a reduction of DA in the caudate putamen complex (CPU). However, histological studies in both humans and animals have demonstrated loss of cells in a number of non-DAergic cell populations in PD, including noradrenergic neurons of the locus coeruleus and dorsal vagal nucleus, serotonergic neurons of the dorsal raphe nucleus (DR), and cholinergic neurons within the substantia innominata and the pedunculo-pontine nucleus \$1-31. Damage to these systems may influence movement-related and non-movement-related aspects of $P D$, such as cognitive function and $\operatorname{mood}[3,4]$.

Approximately $25-40 \%$ of PD patients suffer from depressive disorder [4,5]. Dysfunction of the serotonergic neurotransmitter system is commonly associated with depression 16 . The first clues regarding serotonin (5-hydroxytryptamine, 5-HT) involvement in PD were based on postmortem neurochemical data 17.91 and decreased levels of 5-hydroxyindoleacetic acid (5-HIAA) in cerebrospinal fluid (CSF) in brains of depressed PD patients [10-13]. Initial results were based on data from postmortem research, and thus generally based on patients in late stages of their disease. it is, however, also known that serotonin levels are found to be decreased in earlier stages of PD [14,15]. Serotonergic neurons in the DR and central 5-HT levels are reduced in the parkinsonian brain $|3,16|$, suggesting a link with depressive as well as non-depressive symptoms of PD. Serotonergic efferents, from the DR have been found to innervate both the CPU and the SN [17]. It is, furthermore, known that PD patients may suffer from dysfunction of the hypothalamic-pituitary-adrenal (HPA) axis resulting in abnormal cortisol and ACTH responses [18-20]. Since the serotonergic axis influences the HPA system, the endocrine abnormalities found in PD patients might also be related to deficient functioning of the 5-HT system [21]. These findings have led to the suggestion that 5-HT may play a significant role in the pathophysiology of PD. Furthermore, more knowledge about the exact role of 5-HT in PD may lead to novel treatment strategies for PD, which at present are based on DA and acetylcholine (ACh).

The possible role of 5-HT in PD is described by two opposing hypotheses. The first hypothesis regards 5-HT dysfunction as the primary event in PD [22]. In this hypothesis 5-HT is agonistically linked to DA, i.e., a decrease in 5-HT leads to a decrease in DA. The second hypothesis reglards 5-HT decrease as a secondary event in PD [23-25]. Here it is assumed that 5-HT is antagonistically linked to DA, i.e. a decrease of 5 - HT is a secondary compensatory mechanism for the initial DAergic degeneration and thus reduces the decrease in DA. Circumstantial evidence for this hypothesis can be found in the observation that selective serotonin reuptake inhibitors (SSRI's) may worsen motor symptoms in PD [26]. 
Several animal models have been used for investigating PO (also see chapter I and 3). Unfortunately, there is no general agreement on what is the best method for modeling PD. In the present study a partial billateral striatal 6-hydroxydopamine (6-OHDA) lesion rat model was used. There are several arguments for using a bilateral model instead of the more widely used unilateral lesion model [27,28]. Firstly, PD affects the human brain bilaterally, thus using a bilateral lesion resembles the human situation of PD more than using a unilateral model. Secondly compensating mechanisms from the contralateral side are reduced [29]. Also, in rats with intrastriatal 6-OHDA lesions, it was demonstrated that bilateral 6-OHDA lesions are more suitable for testing behavioral motor paradigms than unilateral 6-OHDA lesions $130 \mid$. In the present study the partial bilateral striatal 6-OHDA lesion was used to investigate the role of DA and 5-HT in an animal model of PD, using pharmacological challenge studies. Following the hypothesis of Mayeux [24] described above, it was expected that DA and 5-HT would have antagonistic effects.

\section{Materials and Methods}

\section{Animals}

23 male Lewis rats (bred in our animal facility, Maastricht University, The Netherlands) being 2 months old and weighing $200-250 \mathrm{~g}$ at the beginning of the study were used. The rats were housed under standard conditions with free access to water but limited food access to reduce their weight by approximately $15 \%[31]$. Animals were housed under a reversed $12 \mathrm{~h}$ light-dark cycle. A radio produced background noise. At the age of 3 months, rats were randomized over 2 groups according to pre test scores achieved in the first month of the experiment on a choice reaction time task. This method created two groups who were equal in performance of the choice reaction time task: the experimental group $(n=14)$ received twofold bilateral injections of 6-OHDA in the center of the CPu and the control group $(n=11)$ received double bilateral injections of saline (sham condition) in the center of the CPu. Prior to surgery and 6-12 weeks post-lesion, several behavioral tests were performed. Table 1 provides an overview of the experimental setup. The present experiments were approved by the animal ethical committee of Maastricht University (The Netherlands) and met governmental guidelines.

\section{Surgery}

The animals which were trained to perform a choice reaction time task, received stereotactic injections of $2 \mu \mid 6-0 H D A(5 \mu \mathrm{g} / \mu /$ dissolved in $0.9 \%$ saline and $0.2 \%$ ascorbic 


\begin{tabular}{|c|c|}
\hline Proporther| & Experimental steps \\
\hline 3 & Training RT task \\
\hline 2 & Training RT task \\
\hline 1 & Training RT task \\
\hline 0 & Surgery \\
\hline +4) & Recovery \\
\hline 2 & Recovery \\
\hline 3 & Recovery \\
\hline 4 & Recovery \\
\hline 5 & Retraining RT task \\
\hline 6 & Testing d-amphetamine $(0 ; 0.1 \mathrm{mg} / \mathrm{kg} \mathrm{BW})$ \\
\hline 7 & Testing $d$-amphetamine $(0.3 ; 1 \mathrm{mg} / \mathrm{kg} \mathrm{BW})$ \\
\hline 8 & Testing ool $(0,0.3 \mathrm{mg} / \mathrm{kg} \mathrm{BW})$ \\
\hline 9 & Testing DOI (11mg'kg BW) \\
\hline 540 & No testing \\
\hline 11 ? & Testing ketanserin $(0 ; 3 \mathrm{mg} / \mathrm{kg} \mathrm{BW})$ \\
\hline 1412 & Testing ketanserin $(10 \mathrm{mg} / \mathrm{kg} \mathrm{BW})$ \\
\hline
\end{tabular}

Table 1. Schematic overview of the experimental setup.

acid; Sigma, Zwijndrecht, The Netherlands) or the same injection amount of the vehicle solution ( $0.9 \%$ saline and $0.2 \%$ ascorbic acid) at four sites (two per hemisphere) in the CPu. One hour before the surgery, rats received desimipramine $(20 \mathrm{mg} / \mathrm{kg}$ in $0.9 \% \mathrm{sa-}$ line, injection volume $1 \mathrm{mg} / \mathrm{kg}$ i.p.).

Desimipramine was given to protect noradrenergic neurons from possible damage by 6-OHDA, and thus make the lesion more DA specific [32]. The animals were anaesthetized using a combination of ketamine $(50 \mathrm{mg} / \mathrm{kg}$ ) and rompun (4 mg/kg) (i.p.) and subsequently placed in a stereotactic frame. The injections of 6-OHDA or vehicle were performed in the CPu at the following coordinates [3i] AP: +0.7 and $-0.4 \mathrm{~mm}, \mathrm{~L}: 2.8$ and $3.4 \mathrm{~mm}$, and DV: $=5.0$ and $-5.0 \mathrm{~mm}$ (from bregma). Injection speed was $0.5 \mu \mathrm{l} / \mathrm{min}$, and the cannula was left in place for an additional 2 minutes before slowly retracting it. Postoperatively, Buprenorfine $(0.2 \mathrm{mg} / \mathrm{kg}$ ) was administered subcutaneous as analgesic. The animals were put back in their home cages for recovery, and weighted and handled daily. The animals had free access to food and water after surgery. Food was pulverized and mixed with water into a porridge, and given inside the cages if rats had feeding problems. Water was consumed without problems. 


\section{Drugs}

Several drugs were tested in a choice reaction time task on the following behavioral parameters: reaction time, motor time, and ratio premature responses. The drugs tested were the partial DA agonist $d$-amphetamine (Sigma, Steinheim, Germany tested at doses $0,0.3$ and $1 \mathrm{mg} / \mathrm{kg}$ body weight (BW)), the $5-H T_{2 \mathrm{a} / 2 \mathrm{c}}$ receptor agonist $1-(2,5-\mathrm{d})$ methoxy-4-iodophenyl)-2 aminopropane hydrochloride (R-(-)-DOl, DOH [34); (Sigma, tested at doses $0,0.3$ and $1 \mathrm{mg} / \mathrm{kg} B W$ ), and the $5-H T_{2 a / 2 \mathrm{c}}$ receptor antagonist ketanserin (135), Tocris Cookson Ltd., Avonmouth, UK; tested at doses 1,3 and $10 \mathrm{mg} / \mathrm{kg} \mathrm{BW}$ ). All drugs were dissolved in $0.9 \% \mathrm{NaCl}$ and injected i.p. 30 minutes before test trials started. Injections of $0.9 \% \mathrm{NaCl}$ were allso used as a control condition (as depicted in Figure 2 to 5 ). Although there are substances with higher specificity for the DAergic system, $d$-amphetamine was used in order to make comparisons possible with earlier experiments of our group $[36,37]$. Order and time frame of drug testing are provided in Table 1.

\section{Behavioral tests}

\section{Choice reaction time task}

The reaction time task used in the present experiment was identical to the task used in earlier experiments of our group. For an overview see $[31,36]$. This task provides information about several aspects of choice reaction time responding of rats. The task was always performed at the same time of day.

The following parameters were used to evaluate the responding of the rats:

Reaction Time (RT): the main latency between the onset of the tone and the release of the hinged food tray panel (after pushing it away). It is generally accepted that re. sponse latencies shorter than $100 \mathrm{~ms}$ may not reflect true RT 1311. These latencies were counted as premature responses. Response latencies longer than 1500 ms were not con. sidered to be a task related RT. The RT performance was evaluated in two ways: the mean reaction time of all responses and the mean $R T$ per hold duration.

Motor Time (MT): the mean latency between the release of the hinged food tray panel and the lever press. It was assumed that MT of more than 2 seconds does not reflect true MT [31].

Number of trials: the tatal number of trialls the rats completed in a session of maximum 30 minutes.

Premature Responses (PR): the total number of times that the rat released the hinged panel before the hold duration had elapsed. This gives an indication of the motivation of the animal. In the present study we always used the ratio of PR (i.e. the PR divided by the total number of completed trials, with a maximum of 60 ). When the to 
tal number of completed trials was not 60 this is mentioned in the text. Furthermore, a PR caused the trial to start over again.

\section{Statistics}

The data obtained from the choice reaction time task, concerning RT, MT and PR over time or over increasing doses of drugs were evaluated with a two-factoriall (Time/Dose and Group) analysis of variance (ANOVA) with repeated measures over time or concentration. Student's t-tests were performed to analyze whether the 6mOHDA lesioned group and the control group differed from each other at every time point or dose. As mentioned above, three different parameters were recorded in the choice reaction time task: RT, MT, PR. Also, the mean number of completed trials was analyzed in every session. In all sessions except the one after administering the highest dose of DOI, the rats completed the maximum of 60 trials. To get an indication of the performance over time, the vehicle injections at post-lesion weeks $6,7,8,9,11$, and 12 were analyzed. The baseline values did not differ from the pre-lesion data (data not shown). In order to enhance reliability, the performance at weeks 6 and 7,8 and 9 , and 11 and 12 were averaged. All calculations were carried out using SPSS Version 10 (SPSS Inc, Chicago, USA). $P<0.05$ was considered statistically significant.

Due to a technical problem, data of 6 control animals concerning the ketanserin measures was, unfortunately, lost. Therefore, the data analysis of ketanserin was performed with the 5 remaining control animals.

\section{Results}

\section{Body weights}

After surgery all animals were fed inside the cages, because of problems with eating from the food rack. Weight loss during the first week post-lesion was about $14 \%$ for the 6-OHDA lesioned animals, while the control group only lost $1 \%$ of their body weight in the first week after surgery. After 4 weeks, the control animals weighed about $19 \%$ more than their pre-operative weights, while the lesioned animals only weighed $9 \%$ more than their pre-operative weights. During this period the animals received food ad libitum, and were not tested behaviorally. After these 4 weeks the animals were food restricted again before behavioral testing. During the course of the present experiment the lesioned animals remained lighter than the control animals [Time: $F(2,46)=58.19$, $p<0.001$ ] (Figure 1). 


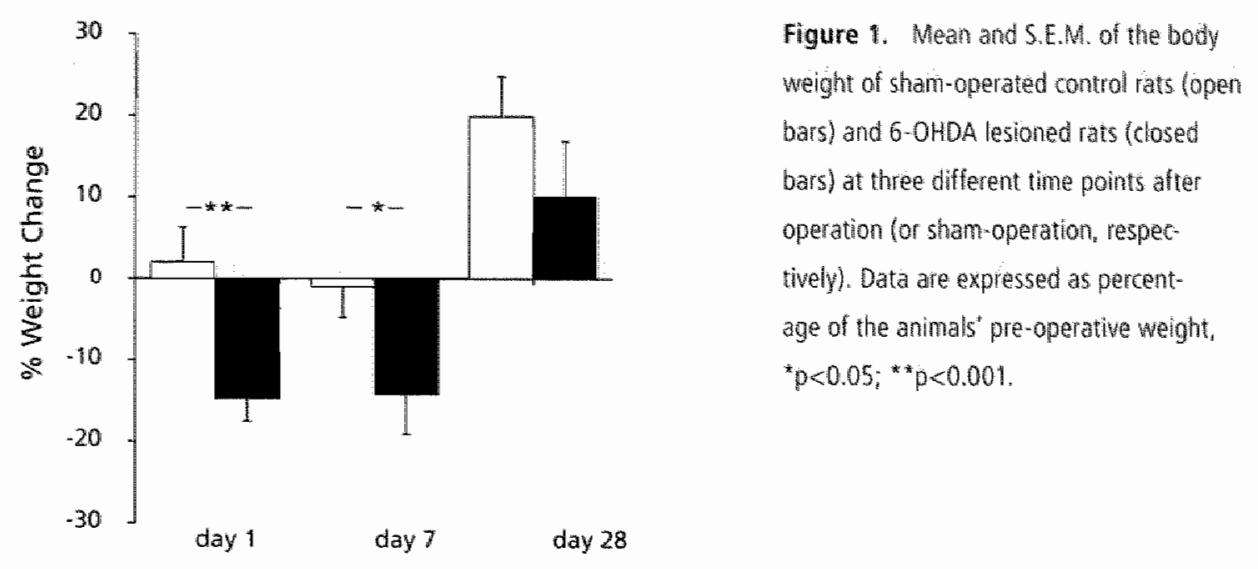

\section{Behaviloral studies}

\section{Baseline performance}

The different substances were tested at different time points during the experiment, as summarized in Table 1. $d$-Amphetamine was tested at weeks 6 and 7. DOl was tested in weeks 8 and 9 , and ketanserin was tested in weeks 11 and 12. In order to obtain an impression of the baseline performance of both groups over the time course of the experiment, the performance of the animals (after the injection of saline) on several measures over time are shown (Figure 2).

Reaction time. The mean RT of 6-OHDA lesioned animals was significantly higher than that of control animals at $6 / 7$ weeks post-lesion $[t=3.22 ; p<0.01]$ and $8 / 9$ weeks post lesion [ $t=4.07 ; p<0.001$ ] (Figure $2 \mathrm{~A}$ ). This effect was not present anymore at 11 and 12 weeks post-lesion (figure 2A). All RTs were also analyzed per hold duration. With increasing hold duration all rats reacted faster. Initially 6-OHDA lesioned rats were slow$\mathrm{er}_{\text {r }}$ this effect was the same at each hold duration (data not shown). Therefore, only the mean RT is always presented.

Motor time. The mean MT of the 6-OHDA lesioned animals was higher than that of the control group at all time points [Group: $F(1,17)=14.36, p<0.01$ ] (Figure 2B). Neither control nor 6-OHDA lesioned animals showed alterations in mean MT over time ITime: $F(2,34)=0.66$, n.s. $]$, therefore, the difference between the two groups remained the same [Group by Time: $F(2,34)=1.35$, n.s.] (Figure 2B).

Premature responses. The mean proportion of PR was significantly higher in the 6OHDA lesioned group than in the control group [Group: $F(1,17)=20.38, p<0.01$ ] (Figure 20). The ratio of the PR changed neither for the control nor for the 6-OHDA lesioned group over time [Time: $F(2,34)=1.56, n . s$.$] . The difference between both groups re-$ mained the same [Group by Time: $F(2,34)=0.20$, n.s.] (Figure $2 \mathrm{C}$ ). 

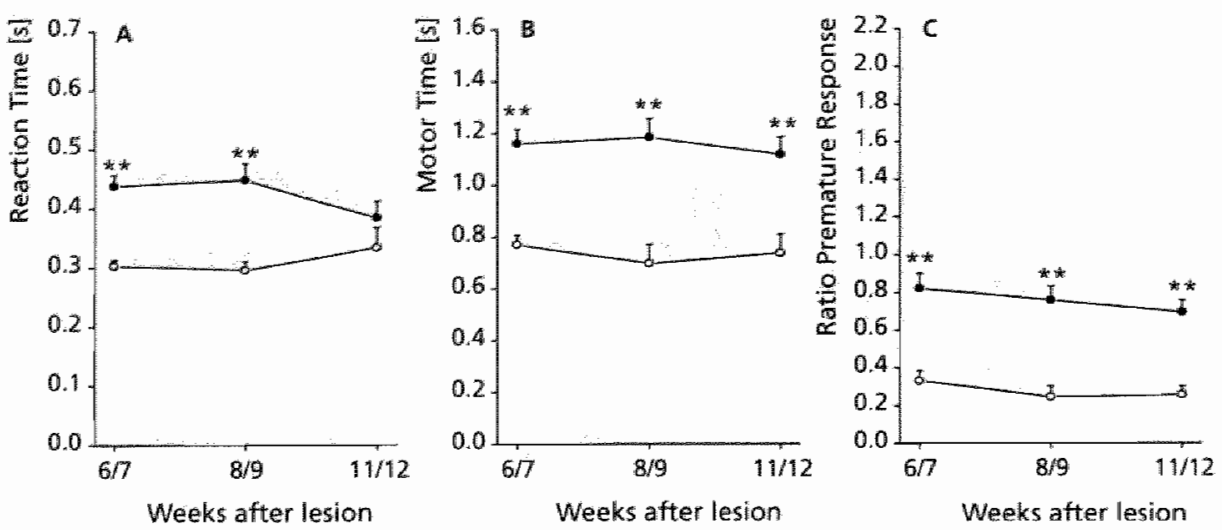

Figure 2. Mean and S.E.M. of baseline performance ( $A$, reattion time; $B$, motor time; $C$, ratio premature responses) of sham:operated control rats (open dots) and 6-OHDA lesioned rats (closed dot5) as a function of the time interwal after operation (or sham-operation, respectively). Data from respectively weeks 6 and 7, weeks 8 and 9 and weeks 11 and 12 were pooled. ", p<0.05; *,$p<0.01$ (differences between sham-operated control rats and 6-OHDA lesioned rats).
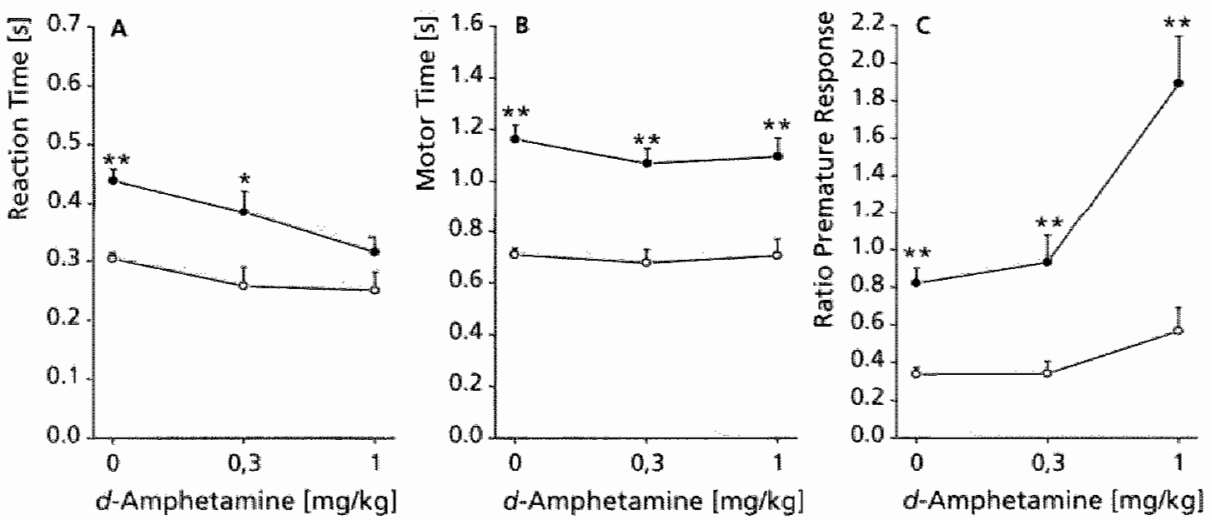

Figure 3. Mean and S.E.M. of reaction time (A), motor time (B) and ratio premature responses (C) of shamoperated control rats (open dots) and 6-OHDA lesioned rats (closed dots) as a function of the dose of damphetanine at 6 and 7 weeks after operation (or sham-operation, respectively) ${ }^{*}, p<0.05 ; * *, p<0.01$ (differences between sham-operated control rats and 6.0HDA lesioned rats). 


\section{d-Amphetamine}

Effects of the partial DA agonist d-amphetamine were tested at increasing doses 10 ; $0.3 ; 1 \mathrm{mg} / \mathrm{kg} \mathrm{BW}$ ) at 6 and 7 weeks post-lesion.

Reaction time. The mean RT in 6-OHDA lesioned and control animals differed significantly [Group: $F(1,21)=10.19, p<0.01]$ (Figure $3 \mathrm{~A}$ ). There was an overall reduction of RT at increasing doses of d-amphetamine [Dose: $F(2,42)=19.68, p<0.001]$. However, the effect was more pronounced in the 6-OHDA lesioned group when compared with the control group [Dose by Group: $F(2,42)=3.69, p<0.05]$. t-Tests revealed that the performance of the groups significantly differed after intervention with $0 \mathrm{mg} / \mathrm{kg} B W$ and $0.3 \mathrm{mg} / \mathrm{kg}$ BW (both $\mathrm{t}$ walues $>2.64, \mathrm{p}<0.05)$, but not $1.0 \mathrm{mg} / \mathrm{kg}$ BW $[\mathrm{t}(23)=1.82, \mathrm{n} . \mathrm{s}$.] (Figure 3A).

Motor time. The mean MT of the 6-OHDA lesioned animals was higher than that of the controls [Group: $F(1,21)=28.62, p<0.001$ ] (Figure 3B). Increasing doses of $d$-amphetamine did not change MT in both groups [Dose: $F(2,42)=2.47$, n.5.]. t-Tests showed that the groups differed significantly over all doses (all $t$ vallues $>4.16, p<0.001$ ). In both control and 6-OHDA lesioned rats, administration of d-amphetamine had no effect [Group by Dose: $F(2,42)=0.73$, n.s.] (Figure 38 ).

Premature responses. The mean proportion PR was higher for the 6-OHDA lesioned animals than for controls [Group: $F(1,21)=24.21, p<0.001$ ] (Figure $3 \mathrm{C}$ ). Increasing doses of $d$-amphetamine increased the PR [Dose: $F(2,42)=16.12, p<0.001]$. This effect was more pronounced in the 6-OHDA lesioned animals than in the control animals [Dose by Group: $F(2,42)=6.70, p<0.01]$. $t$-Tests showed that there was a significant difference between both groups at all doses (all t values $>3.29, p<0.01$ ) (Figure 36).

\section{Dol}

Effects of the $5-H T_{2 a} / 2 c$ receptor agonist DOI were tested at increasing doses $(0 ; 0.3$; $1 \mathrm{mg} / \mathrm{kg} \mathrm{BW}$ ) at weeks 8 and 9 post-lesion.

Reaction time. Overall, there was a significant difference between the RT of the 6. OHDA lesioned rats and the control rats [Group: $F(1,19)=8.64, p<0.01]$ (Figure 4A). Increasing doses of DOI appeared to decrease the RT in the lesioned animals while RT in the control group remained at approximately the same level regardless the dose. This was supported by an interaction effect [Dose by Group: $F(2,38)=3.36, p<0.05$ ]. On the other hand, no main effect of dose was found [Dose: $F(2,38)=0.49$, n.5.]. $t$-Tests showed that 6-OHDA lesioned animals were slower than controls at the doses of 0 and $0.3 \mathrm{mg} / \mathrm{kg}$ (both $\mathrm{t}$ values $>3.74, p<0.001$ ), but the performance at a dose of $1.0 \mathrm{mg} / \mathrm{kg}$ was not different $[t(19)=1.19, \mathrm{n} .5$.$] (Figure 4A).$

Motor time. The mean MT of the 6-OHDA lesioned animals was higher than that of the control animals [Group: $F(1,19)=25.97, p<0.001]$ (Figure $4 B$ ). Increasing doses of DOI increased MT in the rats [Dose: $F(2,38)=20.83, p<0.001]$. The effect of DOI was simi- 

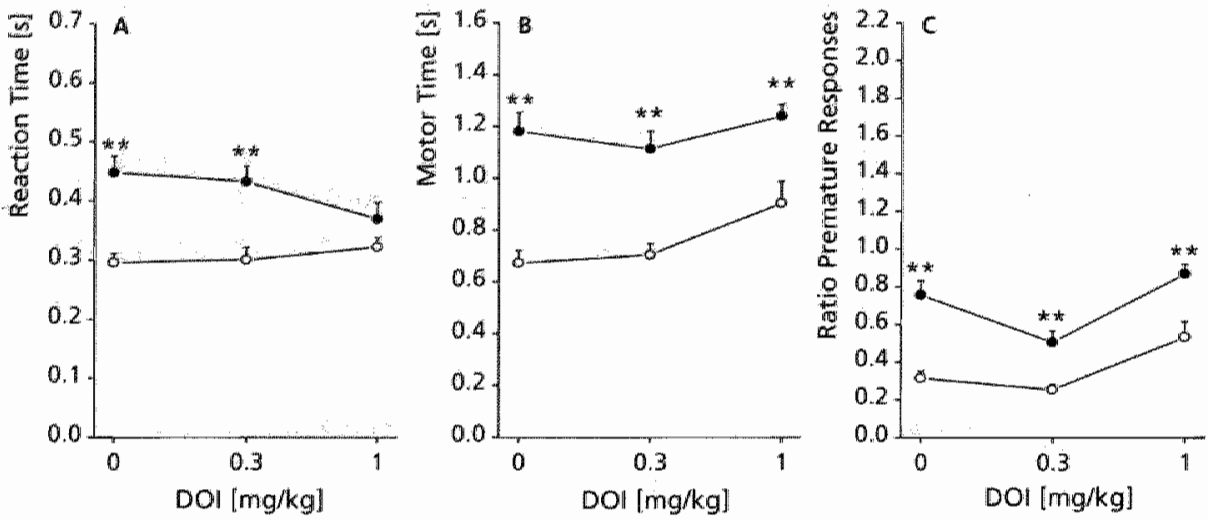

Figure 4, Mean and S.E.M. of reaction tine $(A)$, motor time $(B)$ and ratio premature responses $(C)$ of shamoperated control rats (open dots) and 6-OHOA lesioned rats (closed dots) as a function of the dose of DOI at 8 and 9 weeks after operation (or sham-operation, respectively). ${ }^{*}, p<0.05^{* * *}, p<0.01$ (differences between sham-operated control rats and 6-OHDA lesioned rats).
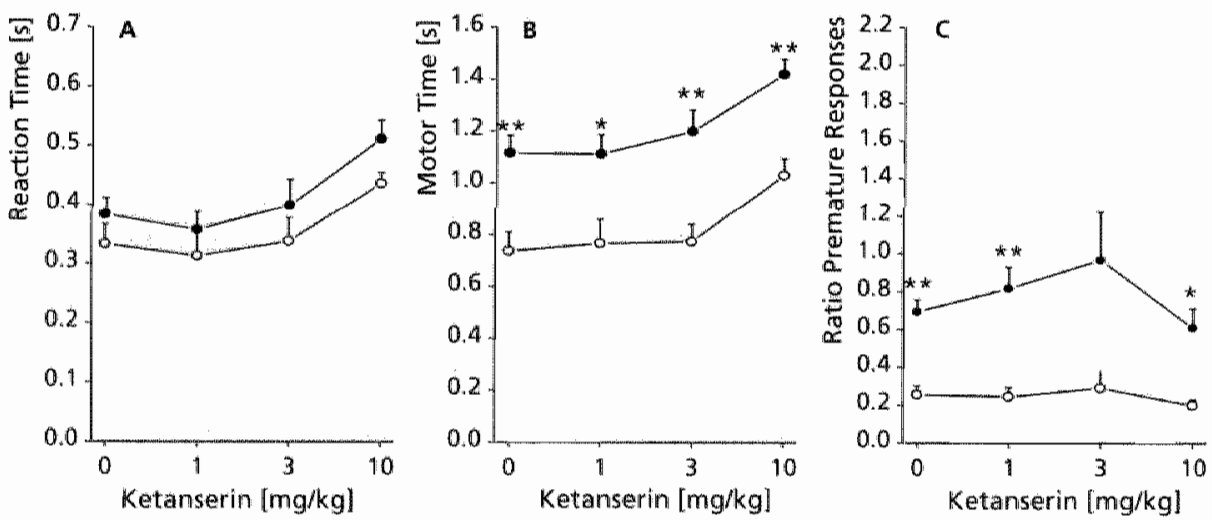

Figure 5. Mean and S.E.M. of reaction time (A), motor time (B) and ratio premature responses $(C)$ of shamoperatad control rats (open dots) and 6-0HOA lesioned rats (closed dots) as a function of the dase of ketanserin at 11 and 12 weeks after operation (or sham-coeration, respectively). ${ }^{*}, 0<0.05_{n}^{* *}, p<0.01$ (differences beween sham-operated control rats and 6. OHDA lesioned rats). 
lar in the control and lesion group [Dose by Group: $F(2,38)=1.44$, n.s.]. t.Tests showed a significant difference between both groups over all doses (all t values $3.90, p<0.01$ ) (Figure 4B).

Premature Responses. The mean ratio PR was higher for the 6-OHDA lesioned animals than for the controls [Group: $F(1,19)=35.19, p<0.001$ ] (Figure $4 \mathrm{C}$ ). Increasing doses of the drug increased the mean ratio of PR [Dose: $F(2,38)=12.22, p<0.001]$. However, DOl did not affect both groups differentially [Dose by Group: $F(2,38)=4,54, n$.s.]. $t$-Tests showed that the groups significantly differed over all doses (all t values $>2.92$, $\mathrm{p}<0.01$ ) (Figure 4C).

Number of trials. Only with the highest dose of the drug DOl $(1 \mathrm{mg} / \mathrm{kg})$, the number of completed trials was affected. Data of three animals from the lesion group had to be excluded (in the highest dose condition) due to the insufficient number of completed trials. After injection of the highest dose both groups showed a reduction of the number of trials completed (52.82 and 46.73 trials for the control and lesion group, respectively, when compared with the maximum of 60 trials which were completed in all other test conditions) [Dose: $F(2,40)=12.16, p<0.001]$, but there was no group difference [Group: $F(1,20)=1.09, n .5]$.

\section{Ketanserin}

The effects of the $5-H T_{2 a}$ /2c receptor antagonist ketanserin were tested at increasing doses $(0,1,3,10 \mathrm{mg} / \mathrm{kg} B W)$ in weeks 11 and 12 after lesioning.

Reaction time. The RT did not differ between the 6-OHDA lesioned animals and the controls [Group: $F(1,14)=1.01$, n.s.] (Figure $5 \mathrm{~A}$ ). Increasing doses of ketanserin worsened RT [Dose: $F(3,42)=13.43, p<0.01]$. Ketanserin had the same effect in both groups [Dose by Group: $F(3,42)=0.17, n .5$.$] .$

Motor time. The overall MT of the 6-OHDA lesioned animals were higher than those of the controls. [Group: $F(1,14)=9.26, p<0.01$ (Figure $5 B$ ). Ketanserin increased the MT. in a dose dependent manner [Dose: $F(3,42)=37.08, p<0.01$ ], and was similar in both groups [Dose by Group: $F(3,42)=0.87$, n.s.]. t-Tests showed that the groups significantly differed at all doses (all t values $>2.52, p<0.05$ ) (Figure 58).

Premature responses. The proportion of PIR was higher in the group of 6-OHDA lesioned animals than in the group of control animals 【Group: $F(1,15)=9.16, p<0.01$ ) (Fig. ure 50). Increasing doses of ketanserim did not affect $P R$ [Dose: $F(3,45)=0.60$, n.s.]. In addition, no interaction effect was observed [Dose by Group: $F(3,45)=0.25$, n.s.] " $t$ Tests showed that the groups significantly differed over the following doses 0,1 , and $10 \mathrm{mg} / \mathrm{kg}$ (all t values $>2.41, \mathrm{p}<0.05$ ). However, after injection of $3 \mathrm{mg} / \mathrm{kg}$ the groups did not differ due to large variations in the 6-OHDA lesioned group $[t(16)=1.54, n .5$.] (Figure 5C). 


\section{Discussion}

The results of the present study indicate that partial bilaterally 6-OHDA lesioned rats exhibit a wide range of behavioral and neurochemical deficits that resemble those observed in patients suffering from mild forms of PD. Impairments were found in motor initiation, motor performance and response inhibition (RT, MT and PR, respectively) in a choice reaction time task. The improvement in RT in the course of the experiment suggested that the 6-OHDA lesioned rats recovered from the lesion. Previously, it has been shown that striatal DA levels need to be decreased by more than $95 \%$ to impair RT [38]. Based on choice reaction time responding after intervention with d-amphetamine, $\mathrm{DOI}$, and ketanserin it can be argued that the $5-\mathrm{HT}_{2}$ system is very likely to be agonistically related to the DAergic system.

Behavioral deficit. The partial bilateral 6-OHDA lesion induced in the terminal site of the nigrostriatal pathway (i.e. $\mathrm{CPu}$ ) caused impairments in all behavioral parameters investigated. Increased RT and MT in 6-OHDA lesioned animals in our choice reaction time task reflect impairments in motor initiation and motor performance, respectively. In a simple reaction time paradigm it has recently been found that $R T$ increases after a bilateral 6-OHDA medial forebrain bundle (MFB) lesion, associated with a decrease of DA in the CPu of $95 \%$ [38]. In the present study the RT of 6-OHDA lesioned rats recovered to normal levels during the post-lesion period at around 11 weeks post-lesion. Thus it may be assumed that after lesioning, the striatal damage in the present experiment was about $95 \%$, at least in the first two months post-lesion.

Compensatory mechanisms, or perhaps regeneration of the damaged structures, might be able to counteract the neurochemical loss produced by the lesion and consequently eliminate behavioral deficits (also see chapter 4) [39]. Results from a study of Eslamboli and colleagues [40] also show recovery of the 6-OHDA model, albeit in marmoset monkeys, indicating that the observed recovery in the present experiment is not unique. Recovery was also observed by Courtière and colleagues in rats with a partial striatal 6-OHDA lesion, and by Tamás and colleagues in rats with a 6-OHDA lesion of the substantia nigra $[41,42]$.

$M T$ and PR of the 6-OHDA lesioned rats were increased and remained at the same level throughout the post-lesion weeks. The increased number of PR in 6-OHDA lesioned rats corroborates findings in studies employing a simple reaction time task [43.44]. This increase might be explained by a higher level of motor activation or preparation which makes the animals react faster than required [44]. The dissociation in recovery between RT and MT/PR indicates that different pathways and brain subregions are responsible for the different components of the choice reaction time task.

Drug effects. I.p. injections of the partial DA agonist $d$-amphetamine at 6 and 7 weeks post-lesion, improved RT of 6-OHDA lesioned rats. As expected, increasing doses 
of the drug resulted in faster RT in 6-OHDA lesioned animals. However, in contrast to our expectations [cf. 36] control rats did not react faster or better to the different parameters of the choice reaction time task at increasing doses of $d$-amphetamine. Most likely, control animals already reacted at an optimal level and the effects of (further increased) $d$-amphetamine were undetectable because of a celling effect.

MT remained unaffected by $d$-amphetamine administration in control and 6-OHDA lesioned animals. There was a clear group difference, but MT levels of both groups did not change following increasing doses of $d$-amphetamine. This was surprising since MT as well as $R T$ were increased by the DAergic lesion. The information processing of motor initiation (i.e. RT) was improved by administering d-amphetamine, but motor performance (i.e. MT) did not benefit from d-amphetamine. Thus again these data support the existence of separate pathways within the basal ganglia which are responsible for both functions. It has to be noted that $d$-amphetamine was injected peripherally and hence the drug may influence other brain sites than the CPu alone. Consequently, DA availability was most probably elevated in all four major DAergic pathways rather than in the nigrostriatal pathway only. Furthermore, d-amphetamine does not only act on DA but also on a1-adrenergic receptors |451. Besides the basal ganglia, motor executive functions are also mediated by cortical pathways. An increase of DA availability in the rat CPu could be beneficial for MT performance in the rats. An additional increase of DA availability in the cortex (via the mesocortical DAergic pathway) might have a negative effect on MT performance. Thus, the overall effect of d-amphetamine might be an unaltered $M T$ in the rats.

$P R$ were similarly increased in both groups at increasing doses of d-amphetamine, as was found in a previous studies $[36,37]$. Interestingly, restoration of the DA availability in the rat $C P u$, by means of $d$-amphetamine injection, did not decrease the number of PR. Instead, the number increased further. This might be explained by the fact that high doses of $d$-amphetamine may cause an over excitation of frontal circuits important for inhibition of inadequate responses.

Increasing doses of DOI improved the RT performance of 6-OHDA lesioned rats, whereas control rats maintained the same performance, most likely due to a floor-effect. After injection of the highest dose, 6-OHDA llesioned animals performed at the same levels as controls. Thus, administration of the $5-\mathrm{HT}_{2 a r 2}$ receptor agonist DOI, resulted in a similar outcome on this behavioral parameter as administration of d-am. phetamine, suggesting that 5-HT and DA are agonistically coupled in the CPU. DOI increased the mean MT in a dose-dependent manner. This effect was observed in both 6-OHDA lesioned animals and control rats. This effect is not likely to be related to sedation or a lack of motivation since the mean RT becaime faster and the proportion of PR increased after DOI administration. The number of PR increased with increasing doses of DOI in both 6-OHDA lesioned and control rats and has been described previously in 
a simillar choice reaction time task $137,46 \%$. This effect of Dol equals again the effect of d-amphetamine administration. This is also in agreement with an agonistic interaction between the 5 HT and DA neurotransmitter systems in the rat brain.

The $5 \sim H T_{2 a / 2}$ receptor antagonist ketanserin was tested in the post-lesion week 511 and 12. At that moment a clear difference in RT performance between the lesion and contral group was not present anymore, most likely due to compensatory and/or recovery mechanisms. However, a significant baseline difference between both groups was. still present for MT and PR at this time point. Increasing doses of ketanserin worsened RT performance in both groups, and all animals had slower RT at increasing doses of ketanserin. This finding is opposite to the effect of the agonist DOI. MT also increased at rising doses of the drug, affecting both the control and the lesioned group in the same way. There was a clear difference between both groups which remained the same regardless of the dose of ketanserin. The increase in MT was not caused by a sedative effect of the drug since there was no impairment in the total number of trials performed in one session of 30 minutes.

At increasing doses of ketanserin PR remained largely unaffected. This is surprising since the 5-HT2a receptor agonist Dal increased the number of PR in both groups, and an opposite response would be expected for ketanserin. This indicates that the relation between cerebral 5-HT and aspects of behavior, like motivation and impulsivity are rather complex.

The partial bilateral striatal 6-OHDA model. PD is slowly progressive in humans, and neurodegeneration of the DAergic and other systems leads to worsening of PD symptoms and increasing loss of DA levels in the dorsal part of the striatum. With regard to the time course, the rat model used in the present study is in contrast with the human condition. When inducing a DAergic lesion in the rat, the rat will go from a state of having an intact nigrostriatal pathway (before the lesion) to a state of having severe neurodegeneration (after the lesion) within a short time period. The rat brain activates compensating mechanisms in response to this neurodegeneration to antagonize neurobiological deficits. It was observed that, over time, the behavioral defects were, to some extent, alleviated in the rat model. This recovery can probably be overcome by injecting 6-OHDA at more locations throughout the $\mathrm{CPu}$, or increasing the dose of 6 OHDA $\mid 30,47 \%$.

PD is a progressive disease with subsequent stages that are characterized by more extensive loss of DAergic neurons and more severe PD symptoms. Since PD is progressive, modeling this disease in the rat implies that the induced syndrome will resemble the human situation at a certain phase of the disease. On the other hand, the activated compensation mechanisms in the rat brain will decrease the severity of the syndrome in the rat, so that, over time, the rat model will resemble earlier PD phases [27]. Although RT showed a recovery over time, this was not observed for parameters such 
as MT and PR. This has also been found in other studies where 6-OHDA lesion models were used $[38,48]$. The recovery of function is possibly due to compensatory mechanisms, like sprouting of remaining DAergic fibers $|49|$. It could also be possible that other neurotransmitter systems (for instance $\mathrm{ACh}$ ) play a significant role in compensation (an overview of the different systems influencing the CPu has been provided in (50)). Also, it should be noted that next to sprouting other compensatory mechanisms (e.g. elevated DA biosynthesis, metabolism, changed receptor levels, and release by the remaining DAergic neurons) could contribute to the recovery of function.

\section{Conclusions}

RT (motor initiation) and MT (motor performance) were impaired in our partial bilateral striatal 6-OHDA lesioned animal model of PD. However, RT was subject to recovery over time. Contrary to RT, MT did not recover. Thus, when using this 6-OHDA model, experiments should only be performed in the first eight weeks after lesioning.

With regard to the role of 5-HT in the 6-OHDA lesioned rat model, $5-\mathrm{HT}_{2 \mathrm{a}}$ and $\mathrm{DA}$ are agonistically linked to each other as based on the finding that the behavioral results of the $5-\mathrm{HT}_{2 \mathrm{a}}$ receptor agonist $\mathrm{DOl}$ and the partial $\mathrm{DA}$ agonist $d$-amphetamine were similar. The behavioral results of the $5-H T_{2 a}$ receptor antagonist ketanserin showed opposing results, which argues further towards an agonistic relation between 5-HT and DA. There was no evidence for a differential effect of the drugs in the two groups of animals. Thus, an agonistic relationship between the 5-HT and DA neurotransmitter systems could be observed in both 6-OHDA lesioned rats and controls. The $5-\mathrm{HT}_{2 \mathrm{a}}$ receptor may play a role in this relation.

The observed agonistic relation between 5-HT and DA was not expected, since in human research an antagonistic relation is generally considered. The present experiments started out from an antagonistic relation [51,52]. DOI and ketanserin also partially, but less strongly, influence the $5-\mathrm{HT}_{2 c}$ receptor subtype. Recently, it has been suggested that $5-\mathrm{HT}_{2 a}$ might have no effect on basal $\mathrm{DA}$ release in the rat $\mathrm{CPu}$, while the remaining antagonistic relation of $5-\mathrm{HT}_{2 c}$ with basal DA release in the CPu should actually be an agonistic one [53]. It should also be noted that the injections were given peripherally, thus other areas besides the $\mathrm{CPu}$ might be influenced.

Contrary to the general consideration in human research, 5-HT and DA appear to be agonistically linked in the 6-OHDA animal model of PD used in the present study. However, the exact role of 5-HT in PD remains unclear, and needs to be examined in further studies. 


\section{Acknowledgements}

The authors would like to thank B. Rutten and W.D.J. van de Berg for their help in com. posing the CPu images, and W. Honig and H.P.E. Steinbusch for their excellent technical assistance.

\section{References}

(1) Jellinger $k$, The pathology of parkinsonism. In: Marsden CD and Fahn 5 (Eds.). Movement Disorders, vol Vol. 2. London: Butterwort $15,1987,124-165$.

[2] Jellinger $k$, New developments in the parhology of Parkinson's disease. Adv Neurol, 199053146.

13) Mohr E, Mendis T and Grimes 3D, Late cognitive changes in Parkinson's disease with an emphasis ofy dementia, Adv Neurol, $19956597-113$.

(4) Curnmings IL, Depression and Parkinsan's disease: a review, Am \& Psychiaty, 1992 149 (4) 443-454.

15] Leentjens Af, Depression in Parkinson's disease: conceptual issues and dinical challenges, I Geriatr Psychiatry Meurol, 200417 (3) 120-126.

16] Van Praag HilM and De Haan S, Central serotonin metabolism and frequency of depression., Psychiatry Res, 1979 $1219 \cdot 224$.

[7] Ehringer $H$ and Honykiewicz $O$, Vertellung von Noradrenallin und Dopamin (3-Hydroxytyramin) im Gehirn des Menschen und ihr Werhalten bei Erkrankungen des Extrapyramidalen Systems, Wien Klin Wschr, 196038 1236. 1239.

[8] Bernheiner $H, B$ irkmayer $W$ and Harnykewicz $O$, Verteilung des 5 -Hydroxytrytamins (Serotonin) im Gehim des Menschen und sein Verhalten bei Patienten mil Parkinson-Syndrom, Wien Klin Wschr, 196139 1056-1059.

(9) Hornykiewicz O, Die topische Lokalisation und das Verhalten von Noradrenalin und Dopamin (3 Hydroxytyramin) in der Substantia Nigra des normalen und Parkinsonkranken Menschen, Wien Klin Wrchr، $196375309-312$.

$|10|$ Johansson B and Roos BE, 5-hydroxyindoleacetic and homovanillic acid levels in the cerebrospinal fiund of healthy valunteers and patients with Parkinson's syndrome, Life Sci, 19676 (13) 1449-1454.

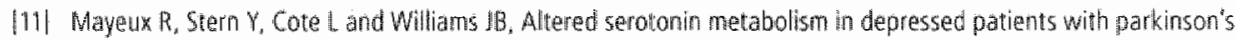
disease, Neurology。1984 $34(5) 642-646$.

[12] Kostic WS, Duricic BM, Covickovic-Sternic N, Bumbasirevic L, Nikoiic M and Mrsujia BB, Depression and Parkinsan's disease: possible role af serotonergic mechanisms, I Neurol, 1987234 (2) 94.96

[13. Kunn W, Muller T, Gerlach M, Sofic E, Fuchs G, Heye N, Prautsch R and Przuntek H, Depression in Parkinson's disease: biogenic anhines in CSF of "de novo" patients, I Neural Transm, 1996103 (12) 1441-1445.

114) Haapaniemi TH, Ahonen A, Tomiainen P, Sotaniemi KA and Myllyla WV, [123\|lbeta-CIT SPECT demonstrates decreased brain dopamine and serotonin transporter levels in untreated parkinsonian patients, Mov Disord, 2001 $16(1) 124-130$

[15] Kerenyi L, Ricaurte GA, Schretlen DJ, McCann U, Warga J "Wathews WB, Rawert HT, Dannals RF, Hilton ${ }_{4}$, Wong DF and Szabo Z, Positron emission tomography of striatal serotonin transporters in Parkinson disease, Arch Neurol, $200360(9) 1223-1229$

[16] Chen CP. Alder IT, Bray L, Kingshury AE, Francis PT and Foster ON, Post-synaptic 5.HTIA and 5-HT2A receptors are increased in Parkinson's dissease neocortex, Ann N Y Acad Sci, 1998861 288-289.

[17] Steinbusch HW, Neuwenthuys R, Verhofstad AA and Wan der Kooy D, The nucleus raphe dorsalis of the rat and its projection upon the caudatoputamen. A combined cytoarchitectonic, immunohistochemical and retrograde 
transport study, J Physiol, 198177 (2-3) 157-174.

If8| Pfeiffer RF, Hsieh HH, Diercks MJ, Glaeske C, Jefferson A and Cheng SC, Dexanethasone suppresion fest in Parkinson's disease. in: Yahr MD and Bergmann KI (Eds). Advances in Neurology, wol k5, New York. Raven Press, 1986, 439-442.

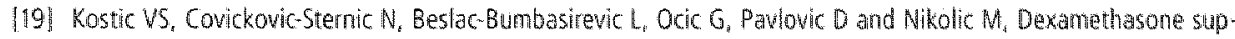
pression test in patients with Parkinson's disease, Mov Disord, 19905 (1) 23-26.

1201 Rabey MM, Scharf M, Oberman Z, Zohar M and GraffE, Cortisol, ACTH, and beta-endorphin after dexamethasome administration in Parkinson's dementia, Biol Psychiatry, 199027 (6) $581-591$.

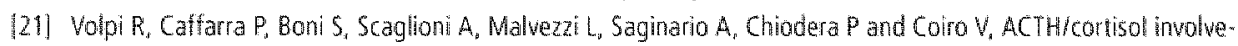
ment in the serotonergic disorder affecting the parkinsonian brain, Neuropsychobiology, 199335 (2) $73-78$.

[22] Steinbusch HW and De Vente], New vistas on the neurobiology of depression. Colocallisation of serotonin-, dopamine-, and nitric oxide synthase-containing neurons in the dorsal raphe nucleus. In: Honig $A$ and Var Praag HM (Eds.), Depression, Willey and Sons, 1997, 179-196.

[23| Hornykiewicz 0, Parkinsoris disease and the adaptive capacity of the nigrostriatal dopamine system: possible neurochemical mecharisms., Adv Neurol $198660140-147$.

[24] Mayeux R, The "serotonin hypothesis" for depression in Parkinson's disease, Adv Neurol, 199053 163-966.

[25] Jacobs BL and Fornal CA, 5-HT and motor control: a hypothesis, Trends Neurosci, 1993 16 (9) 346-352.

[26] Leo RJ, Movement disorders associated with the serotonin selective reuptake inhibitors, J Clin Psychiatry 1996 $57(10) 449-454$.

127) Deumens R, Blokland A and Prickaerts J, Modeling Parkinson's Disease in Rats: An Evaluation of 6-OHDA Lesions of the Nigrostriatal Pathway, Exp Neuroll, 2002175 (2) 303-3:17.

1281 Orth M and Tabrizi 5J. Miodels of Parkinson's Disease, Mov Disord, $200318(7) 729-737$.

[29] Van Dosten RV and Cools AR, Functional updating of the bilateral 6.OHDA rat model for Parkinson's disease, Society far Neuroscience, 1999

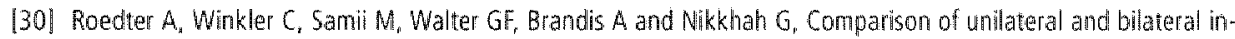
trastriatal 6-hydroxydopamine-induced axon terminal lesions: evidence for interhemispheric functional coupling of the two nigrostriatal pathways, J Comp Neurol, $2001432217-229$

[31] Blokland $A$ and Honig $W$, Intra-striatal haloperidoll and scopolamine injections: effecis on choice reaction tine performance in rats, Eur Neuropsychopharmacol, 19999 (6) 523.531 .

132] Mason ST and Fibiger HC, Neurochemical basis of the dorsal bundle extinction effect, Pharmacal Biochem Be. hav, 197910 (3) 373-380.

133| Paxinos Gand Watson C. The rat brain in stereotaxic coordinates, compact $3 \mathrm{rd} \mathrm{ed.} \mathrm{San} \mathrm{Diego:} \mathrm{Academic} \mathrm{Press,}$ 1996.

134| Sugimoto $Y$ and Yamada I, Effects of 5HT(2A) antagonist 1-(2.5)-dimethoxy-4-iodophenyl-2-aminopropane (DOl) on plasma glucose and glucagon levels of rats, Biol Pham Bull, 200023 (12) 15211523

135] Jorgenson $\mathrm{H}_{4}$ Knigge $U$, Kjaer $\mathrm{A}$ and Warberg J, Adrenocorticotropic hormone secretion in rats induiced by 5 timulation with serotonergic compounds, I Neuroendocrin, 199911 (4) $283-290$.

[36| Blokland A. Reaction time responding in rats, Neurosci Biobehav Rev, 1998 22 (6) 847-864.

137] Blokland A, Sik A and Lieben C, Evaluation of DO1, B-OH-DPAT, eticlopride and anphetamine on impulsive responding in a reaction time task in rats, Beh Pharm, $20051693-100$.

1381 Smith AD, Amalric M, Koob GF and Zigmond MJ, Effect of bilateral 6-hydroxydopamine lesions of the mediat forebrain bundle on reaction time, Neuropsychopharmacolagy, 200226 (6) 756-764.

[39] Bezard $E$ and Grass CE, Compensatory mechanisms in experimental and human parkinsonism: towards a dynamic approach. Prog Neurobial, 199855 (2) 93-116.

[40] Eslamboli A, Baker HF, Ridley RM and Annett LE, Sensorimotor deficits in a unilateral intrastriatal 6-OHDA partial lesion modell of Parkinson's disease in mamoset monkeys, Exp Neurol $2003183418-429$ 

CHAPTER 4

\title{
Recovery after a partial bilateral striatal 6-hydroxydopamine lesion in rats
}

\author{
Bart Scholtissena, Ronald Deumens "Albert F.G. Leentjens ${ }^{a, b}$ "Christoph Schmitza \\ Arjan Bloklanda , Harry W.M. Steinbusch " Jos Prickaerts ${ }^{3}$ \\ alnstitute of Brain and Behaviour, Maastricht University, Maastricht, The Netherlands \\ Department of Psychiatry, Maastricht University Hospital, Mastricht, The Netherlands
}

Adapted from: "Functional investigations into the role of serotonin and dopamine in rats with partial bilateral striatal 6-hydroxydopamine lesions"

\section{Abstract}

The neurotoxin 6-hydroxydopamine (6-OHDA) is widely used for modeling aspects of Parkinson's disease (PD) in animals. A number of different models exist in literature but a bilateral model resembles the human situation closest. However, these bilateral models represent only a minor segment of all 6-OHDA models used. Unilateral models, for instance, are more widely used, but less suited for behavioral testing. The main difference between these models and the human situation is the fact that in humans PD evolves slowly and progressively and shows no signs of recovery, while in animals PD symptoms are produced instantaneously and may show signs of recovery over time. Parallel to the behavioral study described in the previous chapter, a second group of animals received an identical partial bilateral striatal 6-OHDA lesion. At three time points after lesioning ( 3 days, 6 weeks, and 12 weeks) a subgroup was killed in order to obtain a qualitative indication of the extent of the 6.OHDA lesion. Over the three time points, a substantial recovery of tyrosine hydroxylase (TH) staining in the Caudate Putamen complex (CPU) was visible. This implies that this recovery has to be taken into account when using 6-OHDA for modeling PD in animals. 


\section{Introduction}

Parkinson's disease (PD) does not naturally occur in any other species than human beings. Therefore, experimental models of $\mathrm{PD}$ are useful for gaining more insight into possible pathological mechanisms of specific aspects of this disease. Several animal models for investigating aspects of $\mathrm{PD}$ exist 11,2$]$. Unfortunately, there is no general agreement on what is the best method for modeling PD. The discowery of the catecholamine neurotoxin 6-hydroxydopamine (6-OHDA) prowided new options for modeling PD. 6-OHDA is a hydroxylated analogue of the natural dopamine transporter and causes degeneration of nerve terminals and cell bodies 13.51 . Injecting 6-OHDA into the nigrostriatal pathway or directly into the end station of this pathway results in a selective lesion of dopaminergic fibers in the CPu of animals pretreated with desipramine [6]. Not only is the substance with which the lesion is made of great importance, another important aspect is the extent of the lesion.

In the present study a partial billateral striatal 6-hydroxydopamine (6-OHDA) lesion rat model was used. There are several arguments for using a bilateral model instead of the more widely used unilateral lesion model $[2,4]$. First, PD affects the human brain bilaterally, thus using a bilateral lesion resembles the human situation of PD more than using an unilateral model. Second, compensating mechanisms from the contralateral side of the lesion are reduced $17 \%$. Furthermore in rats with intrastriatal 6-OHDA lesions, it was demonstrated that bilateral 6-OHDA lesions are more suitable for testing behavioral motor paradigms than unilateral 6-OHDA lesions [B]. The present pilot study focused on the anatomical and immunohistochemical aspects of the 6-OHDA lesion model of PD.

\section{Materials and Methods}

\section{Animals}

15, 2 months old, male Lewis rats (bred in our animal facility, Maastricht University, The Netherlands) weighing $200-250 \mathrm{~g}$ at the start of the experiment were used. The rats were housed under standard conditions with free access to water and food. Animals were thoused under a reversed $12 \mathrm{~h}$ light-dark cycle. A radio produced background noise. Animals were divided into three groups, and each group of animals received a twofold bilateral injection of 6-OHDA into the center of the CPU. The rats in these groups were killed at the following time points: three days (group 1), 6 weeks (group 2), and 12 weeks (group 3) post lesion. The present experiment was approved by the animal ethical committee of Maastricht University (The Netherlands) and met governmental guidelines. 


\section{Surgery}

The animals received stereotactic injections of $2 \mu \| 6-O H D A(5 \mu g / \mu)$ dissolved in $0.9 \%$ saline and $0.2 \%$ ascorbic acid; Sigma, Zwijndrecht, The Netherlands) at four sites (two per hemisphere) in the (Pu. One hour before the surgery, rats received desimipramine (20 mg/ $\mathrm{kg}$ in $0.9 \%$ saline, injection volume $1 \mathrm{mg} / \mathrm{kg} \mathrm{i}_{\text {. }}$.). . Desimipramine was given to protect noradrenergic neurons from possible damage by 6-OHDA, and thus make the lesion more DA specific $\mid 6 \|$. The animals were anaesthetized using a combination of ketamine (50 mg/kg) and rompun ( $4 \mathrm{mg} / \mathrm{kg}$ ) (i.p.) and subsequently placed in a stereotactic frame. The injections of 6.OHDA were performed in the CPU at the following coordinates $[9] \mathrm{AP:}+0.7$ and $-0.4 \mathrm{~mm}, \mathrm{~L}: 2.8$ and $3.4 \mathrm{~mm}_{w}$ and $D V:-5.0$ and $-5.0 \mathrm{~mm}$ (from bregma). Injection speed was $0.5 \mu \mathrm{l} / \mathrm{min}$, and the cannula was left in place for an additional 2 minutes before it was slowly retracted. Postoperatively, Buprenorfine $(0.2 \mathrm{mg} / \mathrm{kg})$ was administered subcutaneous as analgesic. The animals were put back in their home cages for recovery, and weighted and handled daily. The animals had free access to food and water after surgery. Food was pulverized and mixed with water into a porridge, and given inside the cages if rats had feeding problems. Water was generally consumed without problems.

\section{Immunohistochemistry}

\section{Perfusion and sectioning}

At 3 subsequent post lesion time points ( 3 days, 6 weeks, and 12 weeks) a group of animals was deeply anesthetized and perfused transcardially with somogyi fixative consisting of $4 \%$ depolymerized parformaldehyde, $0.05 \%$ glutaraldehyde, and $15 \%$ picric acid in $0.1 \mathrm{M}$ phosphate buffer ( $\mathrm{pH} 7.4)$. The brains were removed and post-fixed for $2 \mathrm{~h}$ at $4{ }^{\circ} \mathrm{C}$ in the same fixative. After 2 hours, the brains were immersed in $15 \%$ sucrose in $0.1 \mathrm{M}$ phosphate buffer $(\mathrm{pH} 7.4)$ for 24 hours at $4{ }^{\circ} \mathrm{C}$. Subsequently, the brains were frozen with carbogen snow. Coronal free floating sections of $35 \mu \mathrm{m}$ thickness were cut using a cryostat (Leica, Rijswijk, The Netherlands) at the level of the CPu. The CPu sections were stained for $\mathrm{TH}$, and mounted on gelatin-coated slides.

\section{TH immunahistochemistry}

The sections were incubated overnight at $4{ }^{\circ} \mathrm{C}$ with a primary mouse anti-TH antibody (diluted 1:100, generous gift from Dr. C. Cuello, Montreal, Canada). After 3 washing steps (Tris buffered saline (TBS), $0.1 \mathrm{M}$; TBS containing $0.3 \%$ Triton $X-100$ (TBS-T); TBS), the sections were incubated with biotinyllated donkey anti-mouse antibody (Jackson Immunoresearch Laboratories, USA) for 60 minutes at room temperature. After wash. ing, the sections were incubated with ABC-kit (Vector laboratories, USA) for two hours 
at room temperature. Afterwards the sections were incubated with DAB to visualize the Horse Radish Peroxidase (HRP) reaction product. After washing with TBS the sections were mounted on gelatin coated slides, dehydrated and coverslipped for microscopy.

\section{Imaging}

Sections of the CPU in between the two injection sites, showing the highest damage due to the 6 OHDA lesion were visualized with an Olympus BX 51 microscope equipped with a Hitachi HV-C20A camera (Hitachi Kokusai Electronics, Tokyo, Japan) connected to a computer which generated one single montage of multiple, adjacent images with the Virtual Slice application in the Stereolnvestigator software program (MicroBrightfield Inc, Burlington, VT, USA). Final figures were constructed using Corel Draw (Version 11.0; Corel, Ottawa, Canada). Only minor adjustments of contrast and brightness were made, which in no case altered the appearance of the original material.

\section{Results}

\section{Recovery of TH immunohistochemistry}

Injection of 6-OHDA introduced a significant reduction of $\mathrm{TH}$ immunopositive tissue in the CPU (Figure $\| A$ ). This was also observed in other studies [10]. Over the three post lesion periods, however, a clear recovery of the lesioned part of the CPu was observed (Figure $1 B, C$. In the figures, dashed lines indicate the boundaries between unlesioned parts on the left of the CPu and the lesioned parts on the right of the CPU. A gradual decrease in the difference in staining between the unlesioned and lesioned part of the CPU was visible at increasing post leston time points. Direct comparisons of TH labeling intensity

Fïgure 1. Representative photomicrographs of the CPU at thee days (A), 6 weeks (B) and 12 weeks (C) post lesion, showing immunohistochemical labeling for TH. The dashed lines indicate the boundaries between uniesioned parts (on the left) and lesioned parts (on the right) of the CPu. Asterisks indicate unspecific staining of myelin. Insets show TH positive fibers at high magnification in unlesioned parts (on the left) and lesioned parts (on the right) of the CPu. Note the aberrant varicosities in TH positive fibers within the unlesioned part of the CPu ctose to the lesioned part (arrowheads) as well as the complete absence of $T H$ pasitive fibers within the lesioned part at three days $(A)$, as well as the graduat recovery of TH positive fibers within the lesioned part of the CPu with increasing time post lesion (arrows in B and C) $5 \mathrm{cale}$ bar $=100 \mu \mathrm{m}$, and $30 \mu \mathrm{m}$ for the insets. 


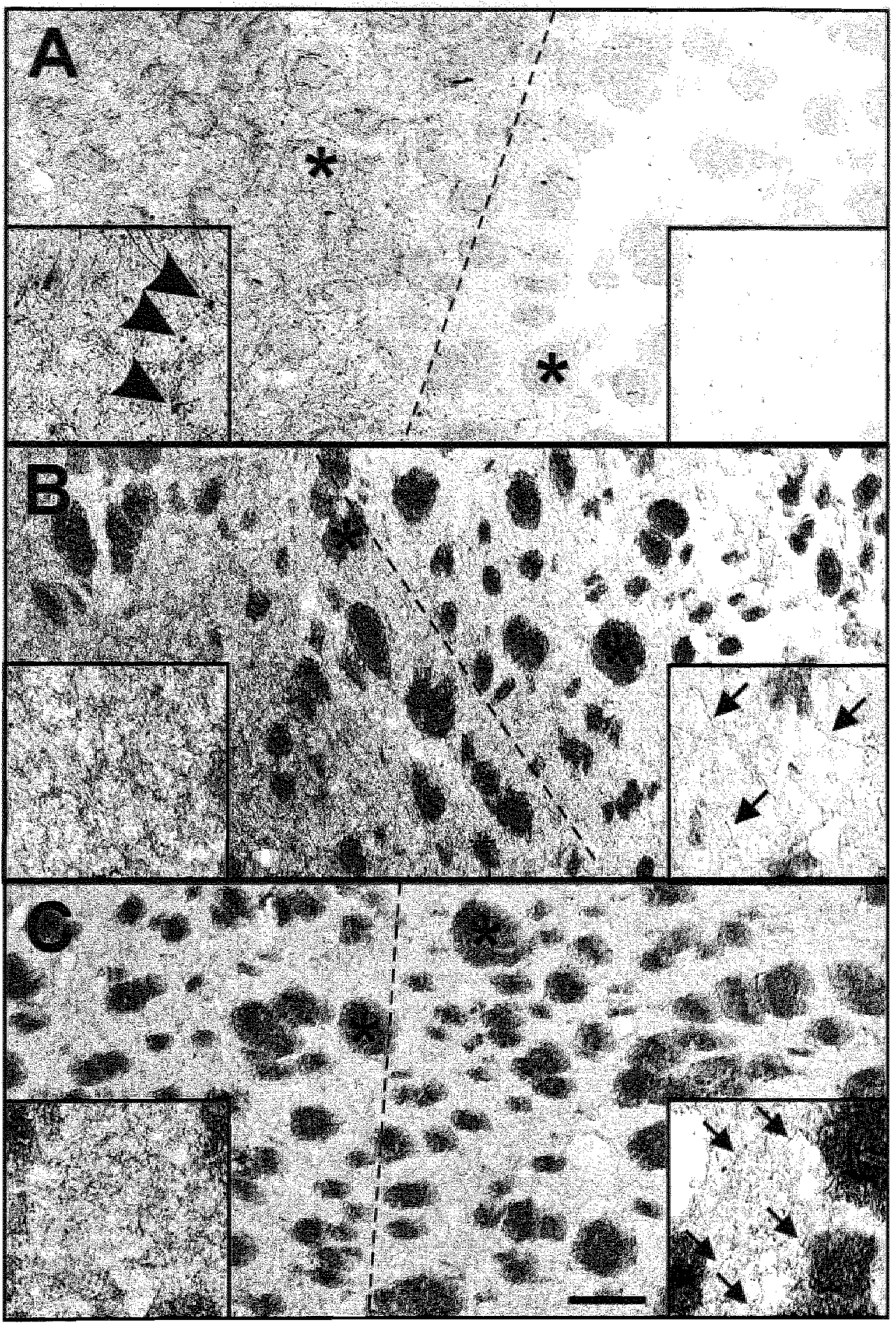


between the investigated time points were complicated by increasing unspecific staining of myelin (indicated by asterisks in Figure 1). Within the unlesioned part of the CPu, close to the lesioned area, unusual varicosities were observed in the TH positive fibers of the CPu (as indicated by arrows in Figure 1). Furthermore, a complete absence of $\mathrm{TH}$ positive fibers within the lesioned part at three days post lesion (Figure $1 \mathrm{~A}$ ), as well as a gradual recovery of TH positive fibers within the lesioned part of the CPu was observed with increasing time after lesion. This recovery of lesioned area in the CPu of animals was paralleled by a partial behavioral recovery as described in the previous chapter.

\section{Discussion}

Over the course of the present experiment a clear recovery of the 6-OHDA lesion was observed, as indicated by TH staining, signifying that the lesion was not permanent. The observed recovery in TH staining was paralleled by a recovery in behavioral parameters, notably reaction time, but not in motor time and premature responses, as described in the previous chapter. Compensatory mechanisms, or perhaps regeneration of the damaged structures, might be able to counteract the neurochemical loss produced by the lesion and consequently eliminate behavioral deficits [11]. Results from a study of Eslamboli and colleagues [12] also showed recovery of the 6-OHDA model, albeit in marmoset monkeys, indicating that the observed recovery in the present experiment is not unique. A behavioral recovery after 6-OHDA lesioning in rats was observed in two other experiments $[13,14]$.

The recovery of the lesioned $\mathrm{CPu}$, which might be caused by collateral sprouting. might provide an explanation for the recovery of the behavioral parameters described above and in the previous chapter. However, since MT did not show signs of recovery, it can be stated that not all aspects of motor behavior as measured in the behavioral experiment described in the previous chapter are dependent on the circuits and structures which were lesioned by the 6-OHDA.

Since recovery of the lesioned area was observed, which can be correlated to the behavioral recovery described in chapter 3 of this thesis, it is important to notice that this recovery limits the usefulness of the model to a specific timeframe. Although the model resembles the human situation closely, it only does this for the period of approximately two months after the lesion has been made. This has to be taken into account in future experiments.

The results described here are to be interpreted with caution. Since this was a pilot experiment, only a small number of animals was included and the staining was only analyzed qualitatively. Although the images provide a clear indication of the status of the lesion, in order to confirm our idea about the anatomical recovery of the lesioned area 
and its influence on the behavioral recovery larger groups have to be used in combina. tion with detailed microscopical and statistical analyses.

\section{Conclusion}

This first qualitative look into the possible anatomical recovery of the 6-OHDA lesion can be seen as the starting point for a more detailed analysis of the subject. From the results of this study, however, it has already become clear that lesion models used for investigating human disease processes have limitations, for instance the time frame within which the 6-OHDA lesion model provides an adequate representation of the clinical situation. When these limitations are taken into account, these models may provide useful information which may lead to the better understanding of disease processes in humans.

\section{References}

(1) Dauer W and Przedborksi 5, Parkinsan's Disease: Mechanisms and Models, Neuron, 200339889.909.

[2] Orth M and Tabrizi SI, Models of Parkinson's Disease, Mov Disord, 200318 (7) 729-737.

[3] Blum D, Torch S, Lambeng N, Nissou M, Benabid AL, Sadoul R and Verna JM, Molecular pathways involved in the neurotoxicity of 6-OHDA, dopamine and MPTP: contribution to the apoptotic theory in Parkinson's disease, $200165(2) 135-172$

[4] Deumens R, Blokland A and Prickaerts 1, Modeling Parkinson's Disease in Rats: An Evaluation of 6-OHDA Lesions of the Nigrostriatal Pathway, Exp Neurol, 2002175 (2) 303-317.

[5] Glinka $Y$, Gassen M and Youdim MB, Mechanism of 6-hydroxydopamine neurotoxicity, $19975055-66$.

[6] Mason ST and Fibiger HC, Neurochemical basis of the dorsat bunde extinction effect. Pharmacol Biochem Ben haw, $197910(3) 373-380$.

[7] Van Dosten RV and Cools AR, Functional updating of the bilateral 6-OHOA rat model for Parkinson's disease. Society for Neuroscience, 1999

(8) Roedter A, Winkler C, Samii M, Watter GF, Brandis A and Nikkhah G, Comparison of wnilateral and bilateral invastriatal 6 -hydroxydopamine-induced axon terminal lesions: evidence for interhemispheric functional coupling of the iwo nigrostriatal pathways, J Comp Neurol, $2001432217-229$

19] Paxinos $G$ and Watson $C$. The rat brain in stereotaxic coordimates, compact $3 i d$ ed, San Diego: Academic Press, 1996.

[10] Smith AD, Amalric M, Koob GF and Zigmond M., Effect of bilateral 6 -hydroxydopamine lesions of the medial forebrain bundle on reaction time, Neuropsychopharmacollogy, 200226 (6) 756-764.

[11] Bezard E and Gross CE, Compensatory mechanisms in experimental and human parkinsonism: towards a dy. namic approach, Prog Neurobiol, 199855 (2) 93-116.

[12] Eslambolli A, Baker HF, Ridley RM and Annett LE, Sensorimotor deficits in al unilateral intrastriatal 6-OHDA partial lesion model of Parkinson's disease in marmoset monkeys, Exp Neurol, 2003183418.429.

[13) Tamas $A$, Lubics $A$, Szalontay $L$, Lengyarl I and Reglodi $D$. Age and gender differences in behavioral and mor 
phological outcane after 6. hydroxydopamine induced lesion of the substantia nigra in rats, $2005158(2)$ 229.

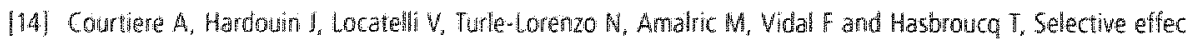
partial stratal 6-OHDA tesions on information processing in the rat, 200521 (7) 1973-1983. 


\title{
Effects of acute tryptophan depletion on cognition, memory, and motor performance in Parkinson's Disease
}

\author{
Bart Scholtissena, Frans R.J. Verhey ${ }^{a, b}$, Jos J. Adam;, Jos Prickaertsa, Albert F.G. Leentjens ${ }^{a, b}$ \\ anstitute of Brain and Behaviour. Maastricht University. Maastricht, The Netherlands \\ Department of Psychiatry, Maastricht University Hospital, Maastricht, The Netherlands \\ Department of Movement Sciences, Mastricht University, Maastricht, The Netherlands
}

\section{Abstract}

Parkinson's disease (PD) is a neuropsychiatric disease which is not only characterized by motor symptoms, but also by cognitive and psychiatric symptoms. It is hypothesized that some of the non-motor symptoms are related to the serotonergic deficiency that is present in PD. The aim of this study was to investigate the influence of serotonin on cognition, memory and motor performance in PD. In a double blind, randomized, placebo controlled, crossover design, the effect of acute tryptophan depletion (ATD) on the Visual Verbal Learning Task (VVLT), the Concept Shifting Task (CST), Simple Reaction Time Task (SRT), Finger Precuing Task (FPT) and the motor section of the Unified Parkinson's Disease Rating Scale (UPDRS, section 3) was investigated in 15 PD patients in early stages of their disease and 15 healthy volunteers, matched for age, sex, and educational status. With the exception of the absence of a differential effect for PD patients with the long interval of the SRT, ATD produced similar effects in PD patients and control subjects on all tasks. These included impairment of delayed recall and delayed recognition on the VVLT, and improved SRT and FPT for 'short intervals'. The UPDRS in patients remained unaffected after ATD. Concluding, serotonin does not appear to play a disease-specific role in cognition and reaction time in early stage PD patients, nor does acute reduction of cerebral serotonin levels affect motor symptoms in a clinically relevant way. 


\section{madrinction}

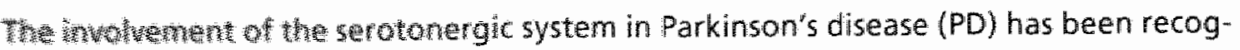

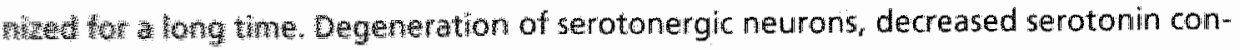

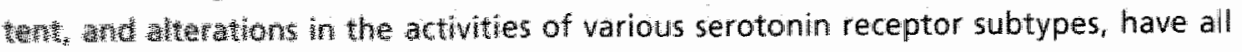
Was

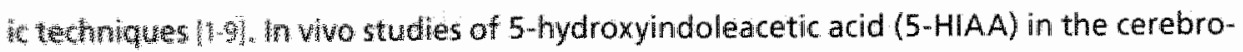

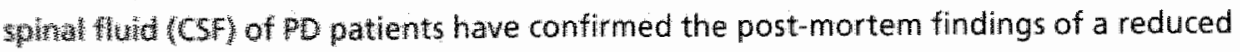
rewothergic tone. Reduced C5F 5-HIAA levels have been described in numerous studies concem $P \mathrm{D}$ (10-12]. The impact of reduced serotonergic activity in $\mathrm{PD}$, however, is still

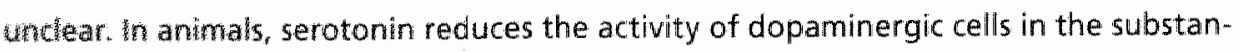
wa niguars compacta and reduces striatal DA release $[13,14]$. Based on these observawons, a compensatory role for the reduced striatal dopamine (DA) activity is hypothesized [s]. Moreover, serotonin activity influences several cognitive functions, such as memory consolidation and attention shifting [16-18].

Recent paradigms have made it possible to study the relationship between cerebral serotonin levels, and cognition and motor behavior in vivo. A widely used method for studying serotonergic function is acute tryptophan depletion (ATD) [19,20]. ATD lowers the level of brain serotonin by depleting the body of its amino acid precursor l-tryptophan (TRP). This is achieved by two mechanisms. First, oral administration of an amino acid mixture dewoid of TRP stimulates peripheral protein synthesis which results in removal of circulating TRP from the blood. Second, the transportation of TRP over the blood-brain barrier is reduced because of a competition between TRP and an abundance of other large neutral amino-acids (LNAA's: valine, leucine, isoleucine, phenylalanine, and tyrosine) for the same active transport mechanism. Once in the brain, tryptophan is synthesized into 5 -hydroxytryptophan (5-HTP) by the enzyme TRP hydroxylase, and this then is decarboxylated by the enzyme aromatic acid decarboxylase into 5-hydroxytryptamine ( $5-H T$, serotonim). ATD, accompanied by a protein free diet results in a decrease of central serotonin levels of up to $80 \% 120,211$. This method of acutely lowering central levels of serotonin has been widely used in healthy and in psychiatric populations to study the role of serotonin in cognition and psychomotor action, but never before in PD patients $\mid 22-27]$.

The aim of the present study was to investigate the role of serotonin in cognitive and motor function in PD patients by temporarly reducing the level of available seratonin in the brain by means of ATD. Based on results from earlier investigations, we hypothesized that ATD might have a negative effect on cognitive performance, but due to a reduced inhibition of striatal dopamine release "as based on results from preclinical dlata, a positive effect on aspects of motor performance. 


\section{Methods}

\section{Subjects}

Fifteen PD patients were included in the ATD study. They were recruited from the neurological outpatient department of the University Hospital Maastricht. All patients were diagnosed with PD, according to the United Kingdom Parkinson's Disease Society Brain Bank (UK PDS BB) criteria [28]. Participation in the study did not have any influence on the medical treatment patients were receiving. Excluded were those patients who were diagnosed with any neurological disease other than PD, or with any psychiatric disorder, including depression, as defined by the criteria of the Diagnostic and Statistical Manual (DSM IV) of the American Psychiatric Association (APA) [29]. This was established in a psychiatric interview. Other exclusion criteria were the use of psychoactive medication, such as antidepressants and antipsychotics, the use of L-dopa, the dopamine agonist lisuride, selegeline, the abuse of alcohol or drugs, and dementia which was defined by a score on the Mini Mental State Examination (MMSE) of less than 23. A prior personai or family history of depression was also considered a ground for exclusion. Control subjects were recruited from an existing database of volunteer subjects of the Maastricht Aging Study (MAAS) [30], and individually matched with the PD patients concerning age, sex and education level. The same in - and exclusion criteria applied to the control subjects, with the exception of PD. The study was approved by the Medical Ethics Committee of the Maastricht University Hospital. All subjects gave their written consent prior to participation and received a financial compensation for participating in the study.

\section{Design}

The study was conducted according to a double-blind, placebo controlled, randomized cross-over design. Intervention consisted of a placebo and TRP depleted mixture. The intervention days were at least 7 days apart in order to rule out any carry-over effects.

\section{Intervention}

The TRP free amino acid mixture consisted of 75 grams of a mixture of fifteen amino acids in the same composition as was used in earlier experiments by our group $|31,32|$. The placebo amino acid mixture was identical in composition, but contained 3.0 grams of TRP. Amino acid drinks were prepared prior to intake by adding $250 \mathrm{ml}$ tap water. Subjects were instructed to consume the mixture as fast as possible. 


\section{Procedure}

All subjects underwent a training session before the actual two test days to control for learning effects. Before this test session the subjects underwent a short physical examination, to rule out any possible physical exclusion criteria. Furthermore, the Hamilton Rating Scale for Depression (HAMD), and the MMSE were obtained to characterize the population [33]. All patients were staged according to the Hoehn and Yahr staging system [34]. On the test days subjects arrived at 9:00 a.m., after an overnight fast. Starting the day, a baseline measurement was obtained after which the subjects received the amino acid mixture.

The measurements were performed again at the point of maximal depletion, which was about $5 \frac{1}{2}$ hours after consumption of the mixture. Blood samples were taken at three times during the day (baseline, 3 , and $5 \frac{1}{2}$ hours after intake of the mixture), in order to check the TRP/LNAA ratio in the subjects' bllood plasma. This ratio can be used as a peripheral measure for central serotonin depletion. Subjects were free to drink water, and were served a protein free bread meal at lunchtime.

\section{Outcome measures}

The Visual Verbal Learning Task (VVLT) and the Concept Shifting Task (CST) were administered to investigate the effects of ATD on cognitive functioning, more specific: learning, memory consolidation and mental flexibillity. Furthermore, motor performance was examined using the Unified Parkinson's Disease Rating Scale (UPDRS) and two reaction time tasks, a Simple Reaction Time task (SRT), and the Finger Precuing Task (FPT). All tasks were obtained at baseline and $5 \frac{1}{2}$ hours after intake of the amino acid mixture.

\section{Visual Verbal Learning Task}

The VVLT is an adapted version of the Auditory Verbal Learning Task [35]. Fifteen words. are presented three times in the same sequence on a computer display. After each of the three trials subjects are asked to recall the words (immediate recall). Twenty-five minutes after the presentation of the first trial subjects are requested to recall as many words as possible (delayed recall). This is followed by a recognition task consisting of 30 words, of which 15 are previously presented and 15 are new but comparable words. The subject has to respond to the words by answering yes or no, indicating the recognition of the words (delayed recognition). Parallel versions were used at different time points.

Outcome variables were the maximum number of words recalled in either of the three immediate recall trials as a measure of immediate recall from short term memory. 
the number of correctly recalled words on the delayed recall as a measure of retrieval from long term memory, and the percentage of correctly recognized words on the recognition task as a measure of long term memory storage.

\section{Concept Shifting Task}

The CST [36] is derived from the Trail Making Test [37] and is used as a measure for cognitive speed, visuomotor tracking and cognitive flexibility (the ability to switch between two concepts, e.g. letters and numbers). The CST consists of five parts. On each sheet, 16 small circles are grouped in a larger circle. In the first three parts the circles contain either numbers (part A), letters (part B), or a combination (part C), all appearing in a random order. The subjects are requested to cross out the items as fast as possible in ascending order in part $A$ and $B$, and in part $C$ continuously change between numbers and letters (e.g. 1-A, 2-B, 3-C, etc.). In the fallowing two parts (01 and 02), the $16 \mathrm{emp}$ ty circles have to be crossed out as fast as possible.

Outcome variables were the interference score , obtained by subtracting the mean time needed for parts $A$ and $B$ (in which the total time needed to perform both parts is composed of a cognitive component and a motor speed component) from the time needed for completing part $C$ (in which the total time needed is composed of the cognitive and motor component from parts $\mathrm{A}$ and $\mathrm{B}$, but also an interference component, since the subject has to change between two strategies) as a measure for cognitive flexibility, and part 02 as a motor score without cognitive interference.

\section{Unified Parkinson's Disease Rating Scale}

The UPDRS [38] is a widely used clinical scale rating several aspects of PD, among which activities of daily living, motor performance, and medication side effects. In the present study only part 3 , the part measuring motor performance, was used.

\section{Simple Reaction time Task}

The SRT [39] is used as a measure of speed of information processing. The subject has to react to a target stimulus which is preceded by a warning stimulus. The subject is instructed to focus his attention on a square on the computer screen in front of him, and press down a button on the table in front of him using his dominant index finger. A warning cue is given (a flashing red square), and after a variable interval the square turns green and the subject has to let go of the button (Reaction Time) and touch the green square as fast as possible (Motor Time). The intervals between target and warning stimulus ranged from 100 to $3000 \mathrm{~ms}$ and were divided into a short (100-1500 ms) and a long (1550-3000 ms) interval. Reaction time and motor time were used as outcome variables. 


\section{Finger Precuing Task}

The finger precuing task is a four choice reaction time task (with the index and middle finger of both hands operating four response keys), where a precue provides information about which fingers to use for responding. That is, the precue reduces the number of possible reactions from four to two by specifying two possible target locations. Subjects have to respond to a single target stimullus, which appears either after a short or a long interval ( 500 and $2000 \mathrm{~ms}$ respectively) after the appearance of the precue signal, by pressing the corresponding button as fast as possible $[40,41]$. Reaction tíme was used as the outcome variable.

\section{Statistics}

This study used a placebo-controlled, double-blind, within-and between-subjects design. Dependent variables of the VVLT, CST were analyzed using a repeated measure analysis of variance (MANOVA), the UPDRS, SRT, and FPT were analyzed using a General Linear Model (GLM) repeated measures design. The $10^{\text {th }}$ version of the Statistical Package for the Social Sciences (SPSS) for Windows was used for the statistical analyses (SPSS Inc., USA). All analyses were performed using delta scores, in order to correct for baseline performance. Within subjects factors were "intervention" (placebo versus ATD) and "interval" (short versus long regarding the SRT and FPT). "Disease" (PD versus control subjects) was entered as between subjects factor.

\section{Results}

\section{Subjects}

Patients (nine men and six women; mean age $61.9 \pm 7.51$ years) had a mean MMSE score of $28.9 \pm 1.36$, which did not differ from the mean score of the control group, $27.9 \pm$ $1.58[F(1,28)=0.865, p=0.093]$. Patients had a mean Hamilton Depression Rating Scale (HAMD) score of $2.1 \pm 1.36$, indicating that there was no clinically relevant signs of depression $[42,43]$.

One patient was treated with an NMDA-antagonist, one patient with anticholinergic medication, four patients took dopa-agonists, and eight patients took a combination of the medication described above. Patients had a median score of II on the Hoehn and Yahr scale (range $1-|1|$ ) $\mid 34\}$. The control group consisted of fifteen (nine men and six women; mean age $60.8 \pm 8.84$ years) healthy age, gender and education matched subjects, who were included on basis of the same criteria as the PD patients with the exception of the diagnosis PD. 


\begin{tabular}{|c|c|c|c|c|c|c|}
\hline Gondition & & ATD & & & Placebo & \\
\hline Time (hrs): & 0 & 3 & 6 & 0 & 3 & 6 \\
\hline \multicolumn{7}{|l|}{ PD patients } \\
\hline Plasma TRP/LNAA & 0.072 & 0.011 & 0.012 & 0.074 & 0.106 & 0.094 \\
\hline Change in ratio $(\%)$ & & -85 & -83 & & 43 & 27 \\
\hline \multicolumn{7}{|l|}{ Control Subjects } \\
\hline Plasma TRP/LNAA & 0.074 & 0.026 & 0.17 & 0.079 & 0.107 & 0.084 \\
\hline Change in ratio (n) & & -65 & .77 & & 35 & 6 \\
\hline
\end{tabular}

Tablle 1. PUasma TRPALAA ratios for PD patients and control subjects in the active and placebo condintion.

\section{Acute tryptophan depletion}

Statistical analysis revealed a significant effect of "intervention" for both groups $[F(1,11)=132.47, p=0.001 ; F(1,12)=17.15, p=0.001$, regarding patients and controls respectively], but no interaction between "intervention" and "disease". TRP/LNAA ratios (indicating the level of central serotonin) dropped already three hours after intake of the mixture with $85 \%$ for patients and $65 \%$ for controls. After 6 hours patients levels were still $83 \%$ lower than at baseline and controls' levels further decreased to $77 \%$ (see also Table 1). In the active condition a decrease in the plasma TRP concentrations was also observed. Plasma TRP dropped by 64 and $71 \%$ after 3 and 6 hours in PD patients, and by 51 and $71 \%$ respectively in controls. In the placebo condition, a fourfold increase in the TRP concentrations was observed in both groups after 3 hours, but this decreased to approximately twice the baseline concentration after 6 hours. All participants tolerated the mixture well. The only observed side effect was nausea, which was reported by 4 patients and 2 controls.

\section{Visual Verbal Learning Task}

Before the start of the intervention, there were no differences between PD patients and control subjects regarding immediate recall, delayed recall and delayed recognition. There were significant effects for "intervention" regarding the delayed recall $[F(1,28)=5.74, p=0.024]$ and the delayed recognition $[F(1,28)=5.11, p=0.0321$, indicating an impairment of performance after ATD. The main effect for "intervention" regarding immediate recall was not significant, but a trend $[F(1,28)=3.5, p=0.072]$. These effects were not qualified by a "intervention" by "disease" interaction, indicating similar effects of ATD on both groups. Immediate recall, delayed recall, and delayed recogni- 

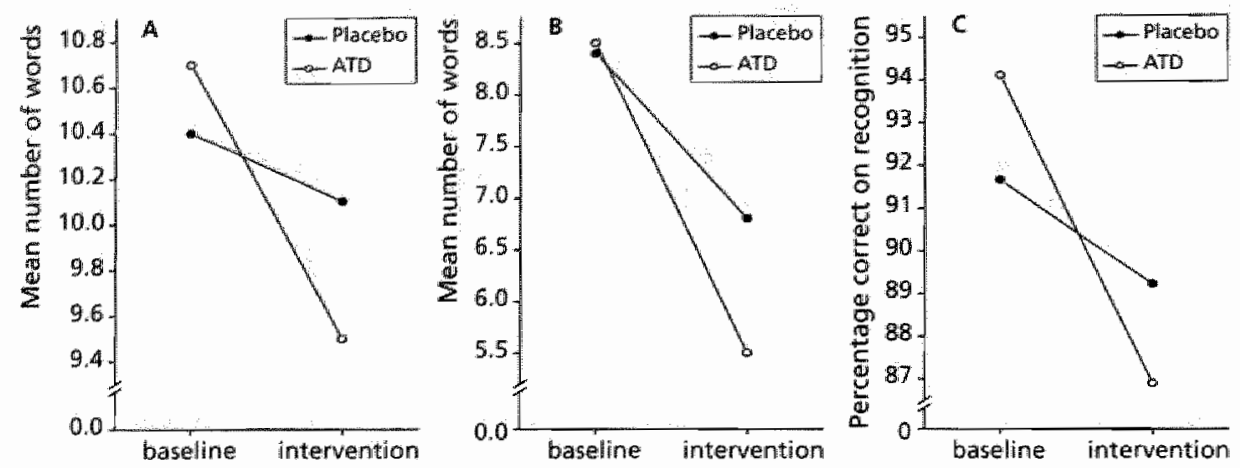

Figure 1. (A) immediate Recall an the WVLT in PD patients in the placebo and ATD condition, (B) Delayed Recall on the VVIT in PD patients in the placebo and ATD condition, and (C) Delayed Recognition in the VVLT in PD pattents in the placebo and ATD condition.
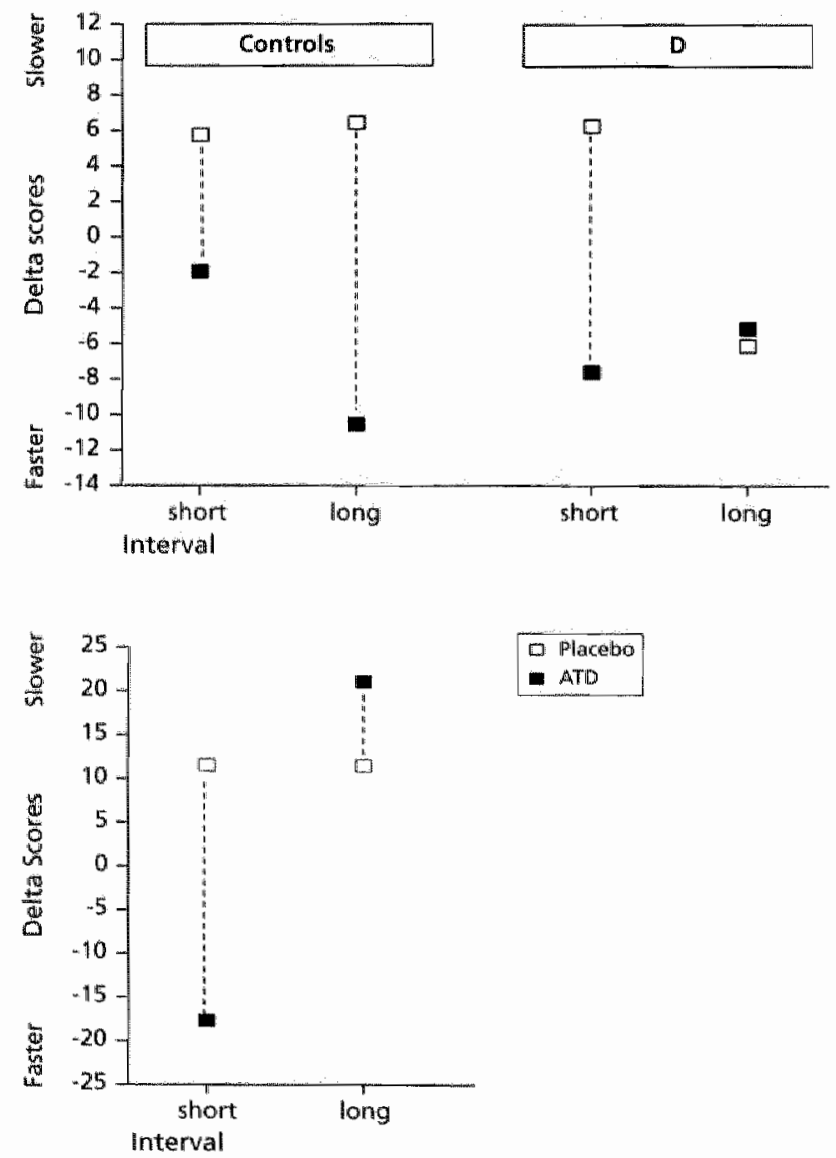

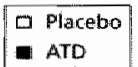

Figure 2. Reaction time performance for patients and controls on the SRT in the placebo and ATD candition.
Figure 3. Reaction time performance in the FPT combined far controls and PD patients in the placebo and ATD condition. 
tion are displayed in Figure $1 \mathrm{~A}, \mathrm{~B}$, and $\mathrm{C}$ respectively. Figures present the combined data from patients and controls.

\section{Concept Shifting Task}

There were no effects for "intervention" regarding both the interference and motor score of the CST. Regarding the interference score, no baseline differences between patilents and controls were observed. A baseline difference concerning the motor part of the CST existed between patients and controls, PD patients performed this part of the CST significantly slower than controls at baseline $[t(28)=-3.78, p=0.001]$, this effect did not change after ATD.

\section{Unified Parkinson's Disease Rating Scale}

No effect for "intervention" was observed. Mean UPDRS scores at baseline were 16.5. Scores dropped both after ATD and after the placebo condition by approximately one point (15.3 after ATD and 15.0 after placebo), indicating no significant change $[F(1,14)=2.39, p=0.145]$.

\section{Simple Reaction Time Task}

There was a significant "intervention" by "interval" by "disease" interaction $\| F(1,28)=$ 9.22, $p=0.049]$ for reaction time, indicating a different reaction on ATD over the short and long interwal for both groups. Further" analysis revealed a "intervention" by "interval" interaction for both groups, indicating that both patients and controls displayed shorter reaction times after ATD $[F(1,14)=4.63, p=0.049 ; F(1,14)=5,52, p=0.034$, respectively for patients and controls]. The effect of ATD was only visible in the short interval for patients, whereas for controls it was visible in both the short and the long interval (see Figure 2). As expected significant baseline differences existed regarding the mo. tor time between the two groups (PD patients and control subjects) on both the short $[t(28)=2.81, p=0.009]$ and the long interval $\llbracket t(28)=2.60, p=0.015 \rrbracket$, with longer motor times for the PD patients than for the control subjects. There were, however, no signifi. cant effects for "intervention", nor were any interactions observed regarding the motor time variable (data not shown).

\section{Finger Precuing Task}

Results of the FPT were comparable to the results of the SRT. There was a significant "intervention" by "interval" effect $[F(1,28)=4.29, p=0.046]$. This effect was not quali- 
fied by an "intervention" by "interval" by "disease" interaction, indicating no group difference. After ATD, both patients and controls showed shorter reaction times on the short interval, but this beneficial effect disappeared for the long interval (see Figure 3).

\section{Discussion}

The aim of the present study was to investigate the effects of ATD on memory processes, cognitive flexibility, and motor performance in PD patients compared to thealthy controls. This was the first study to use the ATD paradigm in a PD population. We hypothesized that acute lowering of TRP and thus lowering of serotonergic availability, might impair memory performance, more specific secondary memory consolidation, in both populations, but might have a greater effect in the PD population since it is known that this group also shows signs of serotonergic degeneration besides the dopaminergic degeneration, even at early stages of the disease $[44,45]$. Based on results from preclinical data, indicating that serotonin has an inhibiting effect on the release of dopamine $[13,14]$, we expected motor performance to improve after ATD.

The patients who participated in the present study were mostly in stage $\|$ of the Hoehn and Yahr staging system, indicating that they were relatively early in the disease process, as is also demonstrated by the fact that none of the patients was using levodopa. Although including only drug-naive patients in a study would be preferred, it is barely possible to include large enough numbers of de-novo patients. Therefore, we decided not to exclude all patients on medication although the use of L-dopa, lisuride or selegeline, was an exclusion criterion. The inclusion of patients in earlier stages of their disease, as well as the use of medication are possible confounders in this study. In general, the acute effects of ATD on cognition and psychomotor performance in PD patients did not differ from the effects observed in the control population, apart from the different reaction on the long interval in the SRT. It is, however, unlikely that the size of the included population is the cause of the absence of differential results between both groups. Since no trends were observed in the results between both groups, it seems unlikely that significant differences would manifest after including higher numbers of subjects.

The observed effects on the VVLT confirm earlier findings of the effect of ATD on cognition in healthy volunteers $[18,32]$. The observed impairment on the delayed recall and delayed recognition after ATD showed that ATD had a relatively specific effect on retrieval of items from long term memory. The fact that a trend was found regarding the immediate recall indicates that ATD might also exert an effect on short term (primary) memory. It is difficult to interpret these results since the cause of the impaired 
long term (secondary) memory aspects might be partially caused by impaired consollidation and storage of the presented words.

Both PD patients and control subjects did not demonstrate any effect of ATD on the CST. Patients were slower on the motor aspects of the task (at baseline, and after ATD), which was to be expected, but there were no differences between both groups regard. ing the interference score. There were no baseline differences between the groups, and an effect of ATD was absent.

The mean UPDRS baseline score was 16.5. Both after ATD and the placebo condition the scores dropped with approximately one point, which does not indicate a significant effect of ATD. This was expected, since the UPDRS is not a very sensitive test to detect small changes in motor performance.

In generall, the reaction time tasks (SRT and FPT) showed similar outcomes. ATD pos. itively affected the reaction times on the short interval for PD patients in both tasks. This advantage was also present for the controls. In controls the reaction time advantage in the SRT task with the short preparation interval was also present for the long preparation interval, while in the patient group it disappeared for the long interval. For the FPT, ATD benefited both PD patients and control subjects, but only with the short preparation interval of $500 \mathrm{~ms}$ and not with the longer preparation interval of 2000 ms. This finding suggests that different preparation mechanisms, mediated trough different pathways, might be active during short and long preparation intervals. Fast, automatic processes are mediating preparation at short intervals, and slower, controlled processes mediate preparation at longer intervals. In PD, the pathway mediating responses after longer intervals was preferentially affected. A similar explanation was given by Marié regarding the differential responses of working memory performance after presentation of information following various delays [46].

\section{Conclusion}

This study was the first to investigate the role of serotonin in cognition and psychomotor aspects in early PD using an experimental approach. We found that the observed effects of ATD on cognition and psychomotor performance in PD patients were not different from the effects of ATD in a healthy control population, with the exception of the absence of a differential effect for PD patients with the long interval of the SRT. This can possibly be explained by the existence of different pathways governing short term and long term responses. In general, serotonin does not seem to play a specific and direct role in cognitive and motor functioning in early PD, other than in healthy control subjects. The results from the present study do not support a compensatory role for serotonin in motor function in early PD, nor do they support a specific role for se- 
rotonin in cognitive functioning in these patients, other than in healthy controls. The fact remains however, that cerebral serotonin is lowered even in relatively early stages of $\mathrm{PD}$, which implies that other hypotheses need to be developed and tested. It may be that serotonin is indirectly inwolved in cognitive and motor processes in PD by controlling the activity of other neurotransmitters, in which case no acute effects from ATD are expected. Another possibility may be that serotonergic degeneration is part of the primary pathophysiology in PD, merely coinciding with dopaminergic degeneration, but without consequences for motor or specific non-motor symptomatology.

\section{References}

(1) Scatton $B$, Javay Agid F, Rouquier $L$, Dubois $B$ and Agird $Y$, Reduction of cortical dopamine, noradrenaline, serotonin and their metabolites in Parkinson's disease, Brain Res, 1983275 (2) 321-328.

[2] Birkmayer $W$ and Riederer $P$, Biachemische veranderungen bei der Parkinson-Krankheit. In: Bírkmayer $W$ and Riederer P (Eds.), Die Parkinson-Krankheit; Biochemie, Klinik, Therapie., Wien: Springer-Verlag, 1985, 29-59.

(3) Birkmayer W and Birkmayer JD, Dopamine action and disorders of neurotransmitter balance, Gerontology, 1987 $33(3-4) \div 68-171$.

[4. Chen CP, Alder JT, Bray L. Kingsbury AE, Francis PT and Foster OV, Post-synaptic 5-HT1A and 5-HT2A rreceptors are increased in Parkinson's disease neocortex, Ann N Y Acad Sci, 1998861 288-289.

15] Cheng AV, Ferrier IN Moris $\mathrm{CM}_{*}$ Jabeen S, Sahgal A, McKeith $1 \mathrm{G}$, Evwardson IA, Perry RH and Perry EK. Cortical serotonin 52 receptor binding in Lewy body dementia, Alzheimer's and Parkinson's diseases, I Neural Sci, $1991106(1) 50-55$.

[6] Maloteaux JM, Laterre EC, Laduron PM, Navoy-Agid F and Agid $Y$, Decrease of serotonin-S2 receptors in temporall contex of patients with Parkinson's disease and progressive supranuclear pallsy, Mov Disord, 19883 (3) 255 262.

7) Perry EK, Perry RH, Candy IM, Fairbaim AF, Blessed G, Dick DI and Tomlinson BE Cortical serotonin -52 receptor binding abnarmalities in patients with Alzheimer"s disease: comparisons with Parkinson"s disease Neurosch Lett 198451 (3) $353-357$.

|8| Castro ME, Pascual J, Romon T, Berciano J, Figols I and Pazos A, 5-HT1B receptor binding in degenerative move. ment disorders, Brain Res, $1998790(1.2)$ 323-328.

(9) Halliday GM, Blumbergs $P C$, Cotton RG, Blessing WW and Geffen LB, Loss of brainstem serotonin and substance P-containing neurons in Parkinson's cisease, Brain Res, 1990510 (1) 104-107.

(10) Lohansson B and Roos BE, 5-hydroxyindoleacetic and homovanillic acid levels in the cerchospinal fluid of healthy wolunteers and patients with Parkinson's syndrome, Life Sici, 19676 (13) 1449-145.4.

I11/ Kuhn W, Muller T"Gerlach M, Sofic E, Fuchs $G$, Heye N, Prautsch R and Przuntek H, Depression in Parkinson's disease; biogenic amines in CSF of "de novo" patients, I Neurai Transm. 1996 103, (12) 1441-1445.

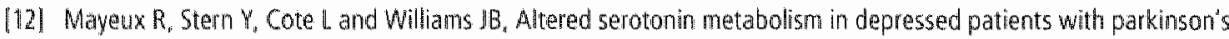
disease, Neurology, 1984 34 (5) 642-646.

[13] Jacobs BL and Fornal CA, 5-HT and notor control: a hypothesis, Irends Neurosci, 199316 (9) 346-352.

[14] Ugedo L, Grentioff 3 and Swenssan TH, Ritanserin, a 5-HT2 receptor antagonist, activates midbrain dopamine neurons by blocking serotonergic inhibition, Psychopharmacology (Berl). 198998 (1) 45-50. 
[15] Mayeux $R$, The "serotonin hypothesis" tor depression in Parkinson's disease. Adu Neurd, $199053163-166$.

$116]$ Buhot MC, Serotonin receptars in cognitive behaviors, Cur Op Neurobial, $19977243-254$.

[17] Buhot MC, Martin 5 and Segu $L_{4}$ Role of serotonin im memory inpaitment, Ann Mad, 200032 (3) 210-22\%.

[18] Rledel W! Klaassen T. Deutz NE, van Someren A and wan Praag HM. Tryotophan depletion in nomal volunteers produces selective impaiment in memory consolidation, Psychophamacology (Berl). 1999141 (4) 362-369.

$119]$ Young SN. Smith SE, Phi RO and Erwin FR, Tryptophan depletion causes a rapid lowering of mood in nomal males, Psychophamacology (Berl), 198587 (2) 173-177.

[20] Reilly JG. Mciawish SFB and Young AH. Rapid tryptophan depletion: a review of studies and experimental methodology, J Psychopharm, 1997 141 362-369.

[21) Carpenter LL, Anderson GM, Pelton GH, Gudin JA, Kiwwin PO, Price L.H. Heninger GR and McDougle CI, Tryptophan depletion during continuous CSF sampling in healthy human subjects, Neuropsychopharmacology. 1998 19 (1) $26-35$.

[22] Kilkens TO, Honig $A$, van Nieuwenhoven MA, Riedel WI and Brummer RJ, Acute tryptophan depletion affects brain-gut responses in irritable bowel syndrome patients and controls, Gut, 200453 (12) 1794-1800.

(23) Booij L. Van der Does Al and Riedel W., Monoamine depletion in psychiatric and healthy populations: review. Mol Psychiatry, 20038 (12) $951-973$.

[24] Gallagher P, Massey AE, Young AE and Hamish MCAillister-Wiliams RH, Effects of Acute Tryptophan Depletion on Executive Function in Healthy Male Volunters, Bill PSychiatry, $20033(10) 1.9$.

[25] Hughes. JH, Gallagher P, Stewat ME, Matthews D. Kelly TP and Young AH, The effects of acute tryptophan de. pletion an neuropsychological function, J Psychopharmacol, 200317 (3) 300-309.

[26] Porter RI, Lunn BS, Waker LL, Gray IM, Ballard CG and O'Brien JT, Cognitive deficit induced by acute tryptophan depletion in patients with Alzheimer's disease, Am J Psychiatry, 2000157 (4) 638-640.

[27] Riedel W], Klaassen T, Griez E, Honig A, Menheere PPCA and Van Pràg HM, Dissociable Hormonal, Cognitive and Mood Responses to Neuroendocrine Challenge: Evidence for Receptor-Specific Serotonergic Qysregulation in Depressed Mood, Neuropsychopharmacology, 200226 (3) 358-367.

(28) Hughes Af; Daniel SE, Kilford $L$ and Lees AJ, Accuracy of clinical diagnosis of idiopathic Parkinson's disease: a clinico-pathologicall study of 100 cases.., Jeurol Neurosurg Psychiatry, 199255 . (3) 181-184.

(29) DSM-IV, Diagnostic ans 5tatistical Manual of Mental Disorders, Washington, DC: American Psychiatric Association, 9994.

(30) Jolles ), Houx P], Van Boxtel MPJ and Ponds R, 1995. The Maastricht Aging Study; determinants of cognitive ag. ing (Neuropsychological Publishers, Maastricht).

[31] Klaassen T. Riedel WJ, Deutz NE, van Someren A and wan Praag HM, Specificity of the tryptophan depletion method, Psychopharmacology (Ber), 1999141 (3) 279-286.

[32] Schmitt JA, Jorissen BL, Sobczak $S_{4}$ van Boxtel MP, Hogervorst E, Deutz NE and Riede! WJ, Tryplophan depletion impairs memory consolidation but improves focussed attention in healthy young volunteers, / Psychopharmacol, $200014(1), 21-29$

133. Folstein MF, Folstein SE and McHugh PR, "Mini-mental state", A practical method lor grading the cognitive state of patients for the clinician, J Psychiatr Res, 1975 , 2 (3) 189-198.

[34] Hoehn MM and Yahr MD. Parkinsonism: onset, progression and mortality, Neurology, 1967 17 (5) 427-442.

135] Rey A. Lexamen psychologique dans les cas deencephalopathie traumatiquet if sychological assessment in cases of traumatic brain injury). Paris: Presses Universaires de France, 1964.

136) Wink $M$ and Jolles $J$, A new wersion of the Trail Maling Test as an information processing task, J Clin Neuropsy, 19857162.

[37] Lezak MD, Neuropsychological Assessment, New York: Oxford University Press, 1995.

[38] Fahn 5 and Elton RL, Unified Parkinson's disease rating scale, New Jersey: McMillan Health Care 1987.

[39] Adarm II, Paas FGWC, Buekers M], Wuyts U, Spikers WAC and Walmeyer P, Perception action couphing in choice 


\title{
The serotonergic hypothesis for depression in Parkinson's disease
}

An experimental approach

\author{
A.F.G. Leentjens $5^{a, b}$, B. Scholtissen ${ }^{b}$, F.W. Vreeling ${ }^{b, c}$, F.R.J. Verhey ${ }^{a, b}$ \\ -Department of Psychiatry. Maastricht University Hospital, Maastricht, The Wetherlands \\ unstitute of Brain and Behaviour, Maastricht Unwersity, Maastricht. The Metherlands \\ Department of Neurology: Maastricht University Hospital, Maastricht, The Netherlands
}

Neuropsychopharmacology (accepted)

\begin{abstract}
The serotonergic hypothesis for depression in Parkinson's disease (PD) states that the reduced cerebrall serotonergic activity that occurs in PD constitutes a biological risk factor for depression. The objective of this study was to assess the serotonergic hypothesis of depression in PD patients using an experimental approach. In a double blind, randomized order, placebo-controlled cross-over design, the response on the Profile of Mood States Questionnaire (POMS) to acute tryptophan depletion (ATD) was studied in 15 PD non-depressed patients and 15 control subjects, without a prior personal or family history of depression. Results indicated that PD patients had lower (worse) baseline scores on the sadness, fatigue and vigor subscales of the POMS, in both ATD and the placebo condition, but not on the tension and anger subscales. There was however no significant between group effect, nor a significant within group effect due to ATD. Concluding, we could find no evidence of a specific serotonergic vulnerability of PD patients for depression. Therefore, our results do not support the serotonergic hypothesis for depression in PD.
\end{abstract}




\section{Introduction}

Mood disturbance, and especially major depressive disorder $r_{f}$ is a common condition in Parkinson's disease (PD), with an average prevalence of $25 \%$ to $40 \%$ in outpatient settings [1]. Depression is associated with a reduced quality of life, increased functional disability, more severe cognitive symptoms, and enhanced caregiver's stress [2-5]. Although there is some knowledge about risk factors associated with depression in PD, little is known about its pathophysiology.

The serotonergic hypothesis is one of the few hypotheses that have tried to link the pathophysiology of PD with an increased risk of depression [6]. This hypothesis is based on three observations. The first is that serotonergic activity is reduced in PD. Indeed, degeneration of serotonergic nerve cellis, decreased brain serotonin content, and alterations in the activities of various types of serotonin receptors, have all been demonstrated in post-mortem studies using neurochemical and autoradiographic techniques [7-9]. Moreover, in vivo studies have consistently demonstrated reduced levels of 5-hydroxyindoleacetic acid (5-HIAA), a breakdown product of serotonin, in the cerebrospinal fluid (CSF) of PD patients $\mid 10,111$, with some studies reporting an additional reduction of 5-HIAA in depressed PD patients $[12,13]$. These findings show the involvement of serotonin in PD. The second observation is the finding in animal studies that serotonin has the ability to inhibit striatal dopamine release [14-16]. This implies that reduction of serotonergic activity leads to less inhibition and a greater dopamine availability. The third is that a reduced serotonergic tone is a known risk factor for depression [17]. Based on these observations Mayeux et al. have formulated the serotonergic hypothesis of depression in PD. This hypothesis considers the reduced serotonergic tone a physiological adaptation to the reduced dopamine activity, while at the same time constituting a risk factor for depression $|6,12|$.

Although there are attractive alternative hypotheses about the role of serotonin in $\mathrm{PD}$, this hypothesis is appealing because it provides an explanation for some common clinical observations. The presence of this biological risk factor for depression may explain the high prevalence of this condition in patients with PD [1]. It may also explain the increased incidence of depression preceding the diagnosis of $P D$, because of the fact that pathophysiological compensatory mechanisms are already in action long before clinical symptoms become apparent [18]. Finally it may provide an explanation for the exacerbation of extrapyramidal symptoms that occur in some PD patients treated with selective serotonin reuptake inhibitors (SSRI's) $[19,20]$. In spite of the fact that this hypothesis was launched in 1984, to date it has not been experimentally verified.

The aim of this study was to test the serotonergic hypothesis of depression in PD in an experimental approach using the acute tryptophan depletion (ATD) paradigm. 


\section{Methods}

\section{Subjects}

Fifteen consecutively referred eligible patients with PD, as defined by the United King * dom Parkinson's Disease Society Brain Bank (UK-PDS-BB) criteria, were included in the study [21].

Criteria for exclusion were the presence of concomitant neurological disorders other than PD, and the presence of concurrent psychiatric disorders, notably major depressive disorder and dementia, as defined by the criteria of the DSM IV [22]. The presence of these psychiatric disorders was assessed in a clinical interview. A prior personal or family history of major depressive disorder, as defined by DSM IV criteria, were also considered exclusion criteria. This was done with the intention of excluding patients with pre-existing risk factors for depression that are not associated with PD-related neurotransmitter changes. A prior personal or family history of depression are known independent risk factors for depression in the general population, that also play a role in patients with PD, and inclusion of patients with these risk factors would confound the recognition of a specific vulnerability related to the pathophysiology of PD [23]. For the same reason, as well as to ensure optimal cooperation with and reliability of the procedure, patients with dementia, as well as those with a score lower than 23 on the Mini Mental State Examination (MMSE), were excluded [24]. Patients currently using psychopharmacological medications could not participate. Although ideally only drug-naive, de novo patients should be included, this was not considered feasible. For pragmatic reasons the use of antiparkinsonian medication as such was not considered a ground for exclusion, except for preparations with a known strong interference with the serotonergic neurotransmitter system, such as levodopa preparations, lisuride and selegeline. Patients on stable doses of dopamine-agonists or anticholinergics were included. No patients were taken off any medication for the sake of the study.

The PD patients were individually matched for sex, age, and educational level with healthy control persons from an existing bank of volunteer subjects of the Institute of Brain and Behaviour of Maastricht University, to which the same exclusion criteria were applied.

Prior to participation, the general health of the subjects was ascertained by physical examination, screening blood tests, and an electrocardiogram. Moreover, an MMSE and a Hamilton Depression Rating Scale (HAMD) were administered, to assess the patients" cognitive and affective status $[25 \mid$. If these investigations revealed additional grounds for exclusion, the patient could not participate. Finally, the patient was classified according to the Hoehn and Yahr (H\&Y) staging system, in order to describe the global severity of PD [26]. 
Pattents were given a verbal explanation and written information of the study and the procedure. All participants gave their written informed consent. Our hospitals" Medical Ethics Committee approved the study.

\section{Design}

The experiment was designed as a double blind, placebo-controlled, randomized order, cross over study. Every subject underwent the ATD procedure twice: once with an amino acid mixture without tryptophan (the active condition), and once with an amino acid mixture containing a balanced amount of tryptophan (the placebo condition). These interventions were performed in a randomized order and spaced at least one week apart in order to exclude carry-over effects. Thus, both a within subject comparison between the active and placebo condition, as well as a comparison between PD patients and controls became possible.

\section{Amino acid mixtures}

The preparation and composition of the amino acid mixture was the same as described by Riedel et al. and Klaassen et al. $[27,28]$ In the active condition, $3 \mathrm{~g} / 100 \mathrm{~g}$ tryptophan was left out. Apart from the amino acids, the mixture contained $63 \mathrm{~g}$ carbohydrates and $33 \mathrm{~g}$ fat in order to dissolve the amino acids and provide caloric value. On both test-occasions, the subjects ingested $75 \mathrm{~g}$ of amino acid mixture, dissolved in 250 milliliters of water.

ATD reduces the availability of tryptophan, the precursor of serotonin, in two ways. Firstly, protein synthesis is stimulated, which uses circulating tryptophan and reduces serum tryptophan levels. Secondly, tryptophan competes with the large neutral amino acids (LNAAs: valine, leucine, isoleucine, phenylalanine and tyrosine) for active transport over the blood-brain barrier, which results in less tryptophan entering the brain. Both plasma concentrations of tryptophan start falling 2 hours, and CSF levels $2 \mathbb{W} / 2$ hours after ingestion of the amino acid mixture, and reach a minimum after 5 to 7 hours 129.301. As continuous supply and synthesis of serotonin in the brain is necessary to maintain adequate levels of serotonergic transmission. ATD creates a temporary deficiency of serotonin. The clinical and physiological consequences of ATD can be followed over a period of several hours. After the intervention tryptophan levels quickly return to normal upon return to a normal diet [28].

\section{Procedure and measures}

At the days of the intervention, the subjects fasted from midnight. Upon arrival at our department at 9:00 a.m. a baseline amino acid spectrum was obtained, and the 32 item 
abbreviated Dutch version of the Profile of Mood States (POMS) questionnaire was ad. ministered 1311 . This version of the POMS is a measure of mood states that assesses five different qualities of mood: sadness, tension, anger, vigor, and fatigue on a $100 \mathrm{~mm}$ visual analogue scale. Lower scores indicate higher symptom levels. Because of its sensitivity for transient mood changes this questionnaire is more appropriate, and often used, in acute interventional studies than depression scales such as the HAMD or Beck Depression Inventory (BDI), because these last two are designed to measure mood over a longer periad of time (typically one or two weeks) 125,321 . Patients were allowed 30 minutes for ingestion of the amino-acid drink. Three and six hours after the start of ingestion, the POMS was taken again. Subjects were provided a light protein-free lunch at noon.

\section{Power callculation and statistical procedures}

As this is the first study using the ATD paradigm in PD patients a formal power calculation based on previously reported findings of effect sizes and standard deviations (SD) could not be performed. Hence, sample size calculation was based on the effect sizes and standard deviations results of two earlier tryptophan depletion studies involving healthy volunteers, conducted in our department [28,33]. A sample size of 15 persons per group would be adequate for detecting a difference of 1 SD with a power of $80 \%$ in a between-group analysis. Because of two reasons we expected that with this calculation the actual power in our study would be on the safe side. First it was expected that the effect sizes (but possibly also the standard deviations) in PD patients would be greater than in healthy control subjects because of the hypothesized greater vulnerabiity of PD patients. Second, sample size calculation was based on a between-group comparison only, and addition of a within-group comparison within the same multivariate analysis of variance (MANOVA) would further increase the power.

A planned interim analysis was performed after inclusion of five patients and five control subjects in order to evaluate whether ATD would be a feasible intervention in $\mathrm{PD}$, and to ascertain adequate levels of tryptophan depletion |34|.

Demographic variables were compared by Chi-square or Students' t-tests. Testing was always done two-tailed with the level of significance set at 0.05 . The score on the POMS sadness subscale was considered the primary outcome measure. As serotonin is also associated with other disturbances in mood, the other POMS subscales were considered secondary outcome measures. Using a multiple repeated measures MANOVA, the effects of disease, disease by intervention, and disease by intervention by time were analyzed for all POMS subscales. All calculations were performed with the Statistical Package for the Social Sciences (SPSS), version 10.0 (SPSS InC., Chicago 1998). 


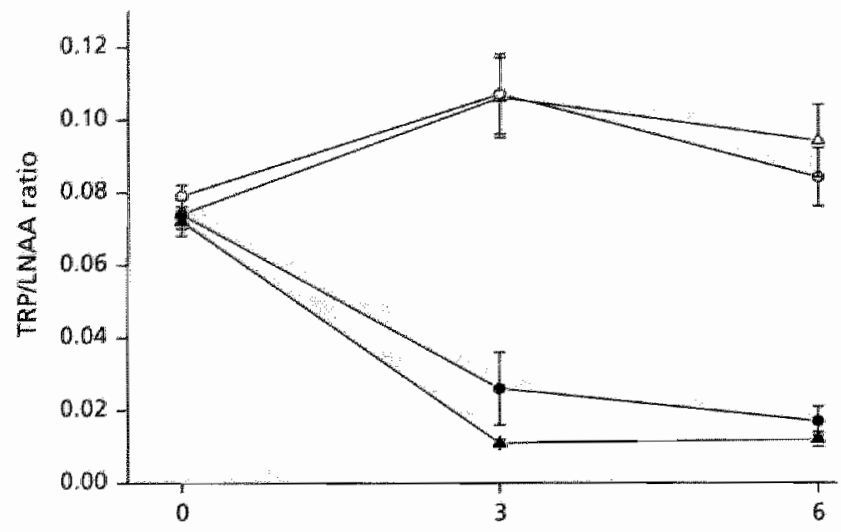

Figure 1. Tryptophand Lapge Neutral Amino Acids (LNAA) ratio during the ATD procedure, at baseline, $t=3$, and $t=6$ hours lflags indicate standard errors).

\begin{tabular}{|c|c|c|c|c|c|c|c|}
\hline Parameter & \multicolumn{2}{|c|}{ PD } & \multicolumn{2}{|c|}{ Control } & 1 & df & p \\
\hline & Average & SD & Average & SD & & & \\
\hline Age & 61.9 & 7.51 & 60.8 & 2.28 & -0.39 & 28 & 0.71 \\
\hline MMSE & 28.9 & 1.36 & 27.9 & 1.58 & -1.74 & 28 & 0.09 \\
\hline HAMD & 2.1 & 1.36 & 0.4 & 0.91 & -4.11 & 28 & $<0.001$ \\
\hline (a $\mathrm{Y}$ (median) & \| & & & & & & \\
\hline
\end{tabular}

Table 1. Demographic characteristics of the participating subjects. SD $=$ standard dewation; MMSE $=$ Mini Mental Status Examination; HAMD = Hamilton Depression Rating Scalle; H\&Y = Hoehn and Yahr staging; $4=t$-test statistics; $d f=$ degrees of freedom; $p=$ significance.

\begin{tabular}{|c|c|c|c|c|c|}
\hline \multirow{2}{*}{\multicolumn{2}{|c|}{1 (hours) }} & \multicolumn{2}{|c|}{ Placebo } & \multicolumn{2}{|c|}{ ATD } \\
\hline & & 0 & 6 & 0 & 6 \\
\hline \multirow{2}{*}{ Depression } & PD & $68(13.1)$ & $72(12.6)$ & $69(93.1)$ & $74(14.5)$ \\
\hline & Contral & $90(7.1)$ & $89(8.9)$ & $90(8.9)$ & $87(10.7)$ \\
\hline \multirow{2}{*}{ Anger } & $\mathrm{PD}$ & $7.4(15.4)$ & $77(13.7)$ & $73(12.5)$ & $77(13.6)$ \\
\hline & Cantrol & $89(8.8)$ & $90(8.2)$ & $90(8.2)$ & $87(12.6)$ \\
\hline \multirow{2}{*}{ Ratigue } & $\mathrm{PD}$ & $59(14.0)$ & $56(15.2)$ & $58(13.7)$ & $65(15,4)$ \\
\hline & Control & $87(10.8)$ & $85(12.4)$ & $87(12.2)$ & $78(177.2)$ \\
\hline \multirow{2}{*}{ Vigor } & PD & $58(15.1)$ & $58(12.5)$ & $57(12.7)$ & $64(16.5)$ \\
\hline & Control & $87(9.5)$ & $81(15.7)$ & $85(11.8)$ & $74(20,4)$ \\
\hline \multirow{2}{*}{ Tension } & $P D$ & $67(13.2)$ & $72(13.7)$ & $69(15.3)$ & $75(14.6)$ \\
\hline & Control & $88(8.3)$ & $90(8.3)$ & $86(11.6)$ & $88(9.1)$ \\
\hline
\end{tabular}

Table 2. Mean scores (SD) on the POM5 subscales for both PD patients and control subjects after ATD and sham depletion at $t=0$, and $t=6$ hours. 


\section{Results}

\section{Participants}

Nine male and 6 female patients with an average age of 61.9 years (SD 7.51) were included in the study. Their average MMSE score was 28.9 (SD 1.4), and their average HAMD score was 2.1 (SD 1.4). The median H\&Y stage was II (average stage 2.4, SD 0.6, range I to III). Two patients were medication free de-novo patients; the other patients were using an NMDA antagonist $(n=1)$, dopamine agonists $(n=4)$ or a combination of both $(n=8)$. Control subjects were individually matched for sex, age and educational level. Thus, there were no significant differences in age and MMSE score. There was however a significant, albeit not clinically relevant, difference in HAMD score between PD patients and controls that was due to higher scores of PD patients on some of the somatic symptoms of this scale. No patients nor control subjects had a prior personal or family history of depression. The demographic characteristics are summarized in Table 1.

\section{Procedure}

All subjects tolerated the procedure well. At the time of the interim analysis, there were no gross clinical changes in motor, affective and cognitive symptomatology that would make continuation of this study unethical. Apart from the nausea, that occurred in 4 patients and 2 control subjects, there were no adverse events. In both PD patients and controls a significant reduction of serum tryptophan was achieved during the active procedure, but not during the placebo procedure. After three hours, the ratio of tryptophan to other LNAA (trp/LNAA) in patients had fallen with $85 \%$, from $7.2 \%$ to $1.1 \%$. In control subjects the trp/LNAA ratio had fallen with $65 \%$ from $7.4 \%$ to $2.6 \%$ in the active condition. These rates of depletion were maintained until after the second set of measurements at 6 hours. In the placebo condition there was a slight increase in trp/LNAA ratio. The effect of ATD is visualized in Figure $t$.

\section{Mood}

The scores on all POMS subscales for patients and control subjects during the procedure are tabulated in Table 2. PD patients scored significantly lower (worse) on three of the subscales of the POMS: sadness, fatigue, and vigor, indicating a significant 'disease effect' (for depression $F=6.49, d f=1,28, p=0.017$; for fatigue $F=4.88, d f=1,28, p=$ 0.035 ; for vigor $F=5.82, d f=1,28, p=0.020$ ). No significant 'disease effect' could be found for hostillity and tension (for hostility $F=3.09, d f=1,28, p=0.089$; for tension $F$ $=0.92$, $\mathrm{df}=1,28, F=0.346$ ). There was no significant 'disease by intervention' effect, 
nor a 'disease by intervention by time' effect for any of the POMS subscales. This means that for both patients and control subjects there was no within-group effect of ATD on POMS scores, nor was there any difference in response on the POMS between the two groups.

\section{Discussion}

\section{ATD procedure}

ATD has been extensively used in psychiatry to study the role of serotonin in aspects of mood regulation and cognition. Most studies report a mood lowering effect of ATD in patients at risk of depression, such as patients with a mood disorder in remission, or a family history of depression or bipolar disorder $[33,35.37]$. In vulnerable individuals, ATD also exacerbates anxiety, panic and aggression (38-40).

In PD patients ATD has so far not been used to study the role of serotonin in mood or other symptom areas. Perhaps this is due to the negative experience of ATD in the only case history of a PD patient described so far $[41]$. In this case history, the patient experienced a serious exacerbation of motor symptoms as well as emergence of depressive symptoms and a significant bradyphrenia occurred during the ATD procedure. All of these symptoms resolved within 2 hours of discontinuing the testing and ingesting a meal containing a tryptophan supplement. In our study none of these adverse events occurred and adequate levels of tryptophan depletion were achieved. Our experience is that ATD is a feasible paradigm to assess serotonergic function in PD patients.

\section{Mood}

In spite of lower baseline scores on the "sadness" "fatigue" and "vigor' subscales of the POMS, there was no differentiall effect of ATD and the placebo condition within each group, nor was there a difference in response to the interventions between the two groups. With respect to mood, this is in contrast with earlier studies that have used the POMS during ATD to assess mood changes in non-PD subjects at risk of depression because of a positive personal or family history of depression. In our study, subjects with these known risk factors were excluded in order to study a potential risk of depression that would be specifically attributable to PD. We could find no differential responses between PD patients and control subjects that would support such a specific serotonergic vulnerability for depression.

Several potential explanations for these negative findings should be considered. A first possibility would be a possible underpowerment of the study. The difficulties in 
performing an adequate sample size calculation were discussed in the methods' section. However, estimation of requested sample size was performed on the basis of two earlier studies. These studies showed that the POMS is sensitive enough to detect differential responses between study groups in vulnerable individuals even with a lower number of included subjects $[28,33]$. Another possible explanation may be the existence of a floor effect. It may not be possible to further lower serotonergic activity, and thus elicit mood symptoms, in persons with an already diminished serotonergic function, such as is the case in patients with Parkinson's disease. A similar explanation was given by Delgado et al. who reported no additional mood changes during ATD in untreated depressed patients [42]. However, our PD patients were not depressed and thus lowering of mood would be possible as a reaction to ATD. Yet another explanation is the fact that ATD is a method that is especially suitable to demonstrate presynaptic serotonergic dysfunction, while being less sensitive to demonstrate postsynaptic dysfunction. In the case of postsynaptic serotonergic dysfunction the postsynaptic cells may already be less responsive to serotonin anyway [42]. There is some evidence for postsynaptic serotonergic dysfunction in PD. Three interventions assessed serotonergic function in PD patients with different serotonin agonists. Blunted cortisol, $\mathrm{ACTH}$, and prolactin responses to a fenfluramine challenge, and a blunted growth hormone $(\mathrm{GH})$ response to a $5 \mathrm{HT}_{1}$ receptor challenge with sumatriptan have been reported $143-451$. These studies are all indicative of a defective serotonergic control of the hypothalamic-pituitary-adrenal (HPA) axis in PD patients. A limitation of these interventions is that in a design where agonist substances are used, which enhance serotonin avallability, it is not possible to elicit mood symptoms. Hence these designs are not suitable to study the serotonergic hypothesis of depression in PD. With the exception of one pilot study that as sessed postsynaptic $5 \mathrm{HT}_{2}$ a receptor binding in $\mathrm{PD}$, only presynaptic parameters, such as the $5 \mathrm{HT}$ transporter (5HTT) and the $5 \mathrm{HT}_{1 \mathrm{ta}}$ receptor have been studied. In the pilot study $5 \mathrm{HT}_{2 \mathrm{a}}$ receptors were differentially increased and decreased in different brain regions that could not be linked to depression $|46|$.

If we accept that these explanations are unlikely, the only feasible explanation is that, contrary to other vulnerable groups, the known vulnerability for depression in PD patients is not directly related to the reduced serotonergic activity.

Other limitations of this study that ought to be mentioned are the fact that the allowed medication may still have played a confounding role, and the fact that there are no data on validity or reliability of the POMS in patients with PD.

\section{Implications}

In our study, the vulnerability of PD patients for depression cannot be directly linked to a reduction in serotonin activity. It can also be hypothesized that serotonin plays a 
more indirect role as a regulator of other neurotransmitters involved in the pathophysiology of depression, such as dopamine and noradrenalin. Although in men most studies point at an antagonistic interaction between serotonin and dopamine, animal studies also support an agonistic interaction [47]. Some authors suggest that in PD serotonergic degeneration may be primary, and the degeneration of the dopamine system secondary $[48,49]$. Moreover, other neurotransmitters may be more directly related to mood symptoms in PD, such as dopamine. The 'dopaminergic hypothesis for depression in $P D$ ', was formulated by Fibiger in the same year as the serotonergic hypothesis was formulated [50]. He considers the reduced dopamine responsible for the high incidence of depression. Deficiency in this system would lead to malfunction of self-reward systems that would constitute a risk for depression. This hypothesis would also provide an explanation for the high prevallence of depression in PD and the fact that depression may precede PD. It also provides an explanation for the beneficial effects on mood of some of the dopamine-agonists, and for the problem of dopamine dependence that exists in some patients [51-54]. Although formulated in 1984, this hypothesis too is still in need of experimental verification.

The pathophysiological basis of depression in PD may also influence the clinical approach to treatment. In clinical practice the treatment of depression in PD patients, is largely focussed around selective serotonin reuptake inhibitors (SSRI's) or atypical agents such as venlafaxine and mirtazepine. In the Practice guideline for the treatment of major depression in adults of the American Psychiatric Association (APA) SSRI's are mentioned, alongside bupropion, as a first choice treatment for depression in PD [55]. This advice is based on side-effect profiles, but not on efficacy or pathophysiological arguments. The two placebo-controlled trials with an SSRI in PD are characterized by a high placebo response without superior efficacy of citalopram or sertraline respectively $[56,57]$. In a Cochrane review no evidence was found for superior efficacy of any antidepressant over placebo in depressed PD patients [58]. If serotonergic deficiency is not the main pathophysiological mechanism in depression in PD, it may be worthwhile to look at other potential treatment options, including agents that more specifically address the noradrenergic and the dopaminergic system.

\section{Conclusion}

ATD is a feasible research method to assess central serotonergic function in PD. Adequate levels of tryptophan depletion were achieved without clinical adverse effects. No differential response on the POMS subscales were observed between the active and placebo condition within each group, nor was there any difference during the active condition between PD patients and controls. Although serotonin is clearly involved in 
the pathophysiology of PD, our study did not find evidence for a direct relation between serotonergic activity and mood symptoms. Thus, this study provides no support for the serotonergic hypothesis of depression PD. In this light, alternative hypotheses, such as the 'dopaminergic hypothesis' ${ }^{\prime}$ would merit experimental imvestigation. In the cinical practice of treating depressed PD patients, non-serotonergic antidepressants may be interesting treatment options that should be further explored.

\section{References}

11 Leentjens AFG, Depression in Parkinson's disease: conceptual issues and clinical ehailenges. I Ger Psy Neurol, 200417 120-126.

[2] Hobson P. Holden A and Meara ), Measuring the impact of Parkinson's disease with the Parkinson's Disease Quality of Life questionnaire, Age and Ageing, 199928 341-346.

(3) Liu CY, Wang SJ, Fuh $\mathrm{L}$, Lin CH, Vang YY and Liu HC, The correlation of depression with functional ability in Parkinson's disease, INeurol, $1997244493-498$.

(4) Troster Al, Stalp LD, Paolo AM, Fields JA and Koller WC, Neuropsycholological impaiment in Parkinson's disease with and without depression, Arch Neurol, 199552 1164-1169.

15] Aarsland D, Mental symptoms in Parkinson's disease are important contributors to caregiver distress., Int I Ger Psych, 199914 (10) 866-874.

[6] Mayetix $R$, The "serotonergic hypothesis" for depression in Parkinson's Disease, Adw Neurol, 199053163 166.

17) Jellinger KA, Pathology of Parkinson's disease. Changes other than the nigrostriatal pathway. Mal Chem Neuropath, 1991 14 (3) \$53-197.

[8] Scatton $B$, Javoy-Agid F, Rouquier $L$, Dubois $B$ and Agid $Y$, Reduction of cortical dopamine, noradrenaline, serotonin and their metabolites in Parkinson's, disease., Brain Res, 1983275321.328.

(9) Chen CPLH, Alder JT, Bray L, Kingsbury AE, Francis PT and Foster OF, Post-5ynaptic 5-HTla and 5-HT2a receptors are increased in Parkinson's disease neocorlex. Ann N Y AC SC, $1998861288-289$.

1101 Johanson $B$ and Roos B-E. 5-Hydroxyindodeacetic and homvanilic acid levels in the cerebrospinal fluid of healthy wolunteers and patients with Parkinson's syndrome., Li SCi, 19676 1449-1454.

[11] Kuhn W, Muler T, Gerlach M, Sofic E Fuchs $G_{*}$ Heye $N$, Prautsch R and Przuntek, Depression in Parkinson's Disease: biagenic amines in CSF of "de novo" patients., I Neural Trans, $19961031441-1445$.

[12】 Mayeux R, Stern Y, Cote L and Willians BW, Attered serotonin metabolism in depressed patients with Parkinson's Disease., Neurology, $198434642-646$.

[13] Kostic W5, Djuricic BM, Covickowic-Sternic N, Bumbasirevic L, Nikoloc M and Mrsulja BE, Depression and Parkinson's disease: possible role of serotonergic mechanisms. J Neurol, $198723494-96$.

114] Jacobs BL and Fornal $C A, 5-H T$ and motor control: a hypothesis. To Neurosci, 199316 (9) 3.46-3.52.

[15] Gerson SC and Baddessarini R., Motor effects of serotonin in the central nervous system. Li Sci, 1980271435. 1451.

(16) Jenner $P$. Sheehy $M$ and Marsden CD. Noradrenaline and 5 -hydraxytryptamine modulation of brain dopamine function: implications for the treatment of Parkinson's disease., Br J Cin Pham, $198315.2775-2895$.

[17] Van Praag HM and De Haen S, Central serotonergic metabolism and frequency of depression, Psy Res, 19791 219.224 
(18) Leentens AFG, Van den Akker M Metsenakers IFM, Lousberg R and werhey FR. J., Higher incidence of depression preceding the onset of Pakinson's disease: a register study. Mou Dis, 200318 414-418.

[19] Leo R, Mowement disorders assaciated with the serotonin selective reuptake inhibitors, I Cin Psy, 199657 (10) 449.454 .

120| Gerber PE and Lynd LO, Selective sevotonim-reuptake inhibitior-induced movement disorders, Ann Phamacotherapy. $199832692-698$.

121) De Rijl MC, Rocca WA, Anderson DW, Melcon MO, Breteler MMB and Maraganore DM, A population perspertiwe on diagnostic criteria for Parkinson's disease, Age and Ageing, 199748 1277-128\%.

[22] Anerican Psychiatric Association, Diagnostic and statistical manual of mental disorders (DSM-HV-TRI. Washington: American Psychiatric Association, 2000.

123] Leentjens AFG, Lousberg $R$ and Verhey FRI, Markers for depression in Parkinson's disease., 2002106 196-201.

$124 \mid$ Folstein MF, Folstein SE and McHugh PR, Mini Mental State. A practical method for grading the cognitive state of patients for the dinician, J Psy Res, $197512189-198$.

[25] Hamilton M. A rating scale for depression, J Neurol Neuros PSy, $19602356-62$.

[26] Hoehn MM and Yahr MD, Parkinsonism; onsel, progression and mortality, Neurology, 1967 17 427-442.

127| Ritedel W, Klassen I, Deutz NEP, Honig A, Van Someren A and Van Praag HM, Tryptophan depletion in normal valunteers produces selective impairment in memory consolidation., Psychopharmacology. 1999141 (4) 362 369.

[28] Klaassen T, Riedel WJ, Deutz NEP, Van Someren A and Van Praag HM, Specificity of the tryptophan depletion method. Psychopharmacology, 1999 141.1 (3) $279-286$.

[29] Carpenter LL, Anderson GM, Peltan GH, Gudin JA, Kirwin PDS, Price LH, Heninger GR and McDougle CI, Tryptophan depletion during continuous CSF sampling in heal thy human subjects., Neuropsychopharmacology, 1998 $1926-35$.

[30] Williams WA, Shoaf SE, Hommer D, Rawling R and M. L, Effects of acute tryptophan depletian on plasma and cerebrospinal fluid tryptophan and 5-hydroxyindoleacetic acid in normal volunteers., Neurochem, 199972 (4) $1647-1647$.

[31] Molvair DM, Lorr M and Droppleman LF, Manuial for the Proflie of Mood States., San Diego: 1971.

[32! Beck. AI, Ward CH, Mendelson M. Mock J and Erbaugh I, An inwentory for measuring depression, Arch Gen Psy, 19614.561 .571 .

(33) Klaassen T, Riedel WI, Van Someren A, Deutz NEP, Honig A and Vars Praag HM, Mood effects of 24-hours tryp. topjinan depletion in healthy first degree relatives of patients with affective disorders., Biol Psy, 199946489. 497.

(34) Leentigens $A F G_{*}$ Parkinson's disease, depression, and serotonin., Maastrichi: Datwyseluniversitaire Pers Maas. wicht, 2002.

(35) Dolgado PL, Chamey OS, Price LH. Aghajanian GK, Landis. $H$ and Heninger $R$, Serotonin functions and the mechanism of antidepressant action: reversal of antidepressant. induced remission by rapid depletion of plasma tryptophath. Arch Gen Psy, $199047411-418$.

[36] Aberg-Wistedt A, Hasselmark L, Stain-Malmgren R, Apéria B, Kjelliman BF and Mathẻ AA, Serotonergic "vulnerability" in affective disorder: a study of the tryptophan dephetion test and the relationships between peripheral and central serotonin indexes in citalopram-responders. ACl Psy 5 can, 799897 374-380.

137) Sobrzak S, Honig A, Nicolson N and Riedel W, Effects of acute tryptophan depletion on mood and cortisol levels in first-degree relatives of type I and type II bipolar patients and healthy matched controls., Neuropsychophermacology, $200227(5) 834-842$.

138| Klaassen T, KLumperbeek I, Deutz NEP, Van Praag HM and Griez E, Effects of tryptophan depletion on anxiety and on panic provoked by carbon dioxide challenge., Psy Res, 199877 167-174.

(39) Kent JM, Coplan JD, Martinez J, Karmally W, Papp LA and Gorman JM, Ventilatory effects of tryptophan deple- 
tion in panic disorder: a preliminary report. Psy Res, 199664 183-90\%

IA0] LeNarquand DG. Pihl RO, Young SN, tremblay RE, Sequin IR, Palmour RM and Benkelhat C, Typtophan depletion, executive funtetion, and disinhibition in agressive, adolescent males., Natropsychopharmacology. 1998 19 333-341

[41] McCance-Katz EF, Marek KL and Price LH, Serotonergic dysfunction in depression associated with Parkinson's disease, Neurology, $1992421813-1814$.

[42] Delgado PL, Price LH, Miller HL, Salomon RM, Aghajanian GK, henninger GR and Chamey DS, Serotonin and the neunobiology of depression. Effects of tryptophan depletion in dirug free depressed patients., Arch Gen Psy, $199451865-874$

[43\Kostic WS, Lecic D, Doder M, Marinkovic II and Filipovic S, Prolactine and cortisol responses to fenthuramine in Parkinson's disease, Biol PSy, 199640 769-775.

[44] Volpi $R$, Caffarra $P$, Boni S, Scaglioni $A$. Malvezzi L, Saginario A, Chiodera Pand Coiro V, ACTH/contisol involvement in the serotonergic disardef affecting the Parkinsonian Brain. Neuropsychobiology, 199735 73-78.

[45) Volpi R, Caffara P, Scaglioni A, Boni S, Saginario A, Chiodera Pand Coiro V, Defective 5-HT1-receptor-mediated neurotransmission in the control of growth hormon in Parkinson's. Disease. Neuropsychobiology. 19973579 . 83.

[46] Van Kroonenburgh $M$, Leentjens $A$, Verhey $F$, Vreeling $F$ and Troost I, Imaging of serotonim $5-412$ ra receptors in Parkirsson's disease. ) Nucl Med, 200142 (5)(suppl)) 227P.

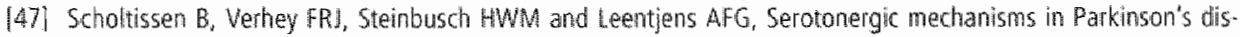
ease: opposing results, from preclinical and clinical data, J Neural Trans, (accepted).

[48] Braak H, Del Treci K, Rub U, De Vos RAl, Jansen Steur ENH and Braak E, Staging brain pathology related to sporadic Parkinson's disease. Neurobiol Aging, 200324 197-211.

[49] Steinbusch HWM and De Vente J, New vistas on the neurobiology of depression: colocalization of serotonindopamin-, and nitric oxide synthase-containing neurones in the dorsal raphe. In: Honig $A$ and Van Praag HM (Eds.). Depression: neurobiological, psychopathological and therapeutic advances., Chichester: Iohn Wiley \& Sons Ltd, 1997.

[50] Fibiger HC. The meurobiological substrates of depression in Parkinson's disease: a hypothesis., Can I Neurol Sci, 198411 (1)(suppi) 105-107.

[51] Corrigan MH, Denehan AQ. Wright CE, Ragual RI and Evans DL, Comparison of pramipexole, fluoxetine, and placebo in patients with major depression. Depression and Anxiety. 200011 58-65.

152) DeBatista C, Solwaon HB, Heilig Breen IA and Schatzberg AF, Pramipexole augmentation of a selective serotonin reuptake inhibitor in the treatment of depression., 200020 (2) 274-275.

[53) Lawrence AD, Evans AH and Lees AJ, Compulsive use of dopamine teplacement therapy in Parkinsonis disease: reward systems gone awry? Lancet Neurol, $20032595-604$.

$154 \mid$ Goldberg JF, K.E. B and Endick CJ, Preliminary randomized, dowble-blind, placebo-controlled trial of pramipexole added to mood stabilizers for treatment resistant bipolar depression. Am j Psych, 2004161 (3) $564-566$.

155] American Psychiatric Association, Practice guideline for major depiessive disorder in adultis. in: (Eds.), American Psychiatric Association practice guidelines for the treatment of psychiatric disorder, Washing ton: American Psychiatric Association, 2000, 413-495.

156. Wermuth L, Sorensen PS, Timm S. Christensen B, Uzon NP, Boas J. Dupont E, Hansen E, Magnussen L, Mikkelsen B. Worm-Petersen J. Lauritzen L, Bayer $L$ and Bech P. Depression in idiopathic Parkinson's disease treated with citalopram., Nor. Psych, $199852163-169$.

[57] Leentiens AFG, Veeling FW, Luijck $G$ J and Verhey FRI, 5SRIs in the treatment of depression in Parkinson's disease, $200318552-554$

1581 Ghazi-Noori S, Chung TH, Deane KHO; Rickards $\mathrm{H}$ and Clarke CE. Therapies for depression in Parkinson's dis. ease., Chichester: John Wiley \& Sons, 2004. 
Table 6.5-Diforevces in the distribution of average wages defined by furw size and industry level (2001)

\begin{tabular}{|c|c|c|c|c|c|c|c|c|}
\hline \multirow{3}{*}{$\begin{array}{l}\text { Skall variables } \\
\text { Characteristics } \\
\text { Wages defined } \\
\text { by skill level }\end{array}$} & \multicolumn{4}{|c|}{ Education } & \multicolumn{4}{|c|}{ occupation } \\
\hline & \multicolumn{2}{|c|}{$\mathrm{Size}$} & \multicolumn{2}{|l|}{ Industry } & \multicolumn{2}{|c|}{ size } & \multicolumn{2}{|l|}{ industry } \\
\hline & Large & medium & Chemical & Metal & large & medium & chemical. & metall \\
\hline \multicolumn{9}{|c|}{ High educated/ white collar high } \\
\hline $10,001-25,000$ & $8.3 \%$ & $41.7 \%$ & $14,3 \%$ & $40 \%$ & $11.1 \%$ & $15.4 \%$ & $15.4 \%$ & \multirow{4}{*}{$\begin{array}{l}11.1 \% \\
77.8 \% \\
11.1 \% \\
0\end{array}$} \\
\hline $5,001-10,000$ & $58.3 \%$ & $33.3 \%$ & $42.9 \%$ & $50 \%$ & $88.9 \%$ & $61.5 \%$ & $69.2 \%$ & \\
\hline $2,001-5,000$ & $33.3 \%$ & $8.3 \%$ & $28.6 \%$ & $10 \%$ & 0 & $23.1 \%$ & $15.4 \%$ & \\
\hline $1,001-2,000$ & 0 & $16.7 \%$ & $14.3 \%$ & 0 & 0 & 0 & 0 & \\
\hline \multicolumn{9}{|c|}{ medium educated/ white collar low } \\
\hline $10,001-25,000$ & 0 & $9,1 \%$ & 0 & $10 \%$ & 0 & & 0 & \multirow{5}{*}{$\begin{array}{l}0 \\
11.1 \% \\
44.4 \% \\
44.4 \% \\
0\end{array}$} \\
\hline $5,001-10,000$ & $8.3 \%$ & $9.1 \%$ & $7.7 \%$ & $10 \%$ & 0 & $8.3 \%$ & 0 & \\
\hline $2,001-5,000$ & $33.3 \%$ & $18.2 \%$ & $23.1 \%$ & $30 \%$ & $55.6 \%$ & $50 \%$ & $58.3 \%$ & \\
\hline $1,001-2,000$ & $58.3 \%$ & $18.2 \%$ & $46.2 \%$ & $30 \%$ & $44.4 \%$ & $41.7 \%$ & $41.7 \%$ & \\
\hline $0,500-1,000$ & 0 & $45.5 \%$ & $23.1 \%$ & $20 \%$ & 0 & 0 & 0 & \\
\hline \multicolumn{9}{|c|}{ low educated/blue collar high } \\
\hline $5,001-10,000$ & 0 & $10 \%$ & 0 & $12.5 \%$ & $0 \ldots$ & 0 & & \multirow{4}{*}{$\begin{array}{l}0 \\
0 \\
100 \% \\
0\end{array}$} \\
\hline $2,001-5,000$ & 0 & $10 \%$ & $7,7 \%$ & 0 & $33.3 \%$ & $20 \%$ & $45.5 \%$ & \\
\hline $1,001-2,000$ & $45.5 \%$ & $20 \%$ & $23.1 \%$ & $50 \%$ & $66.7 \%$ & $70 \%$ & $45.5 \%$ & \\
\hline $0,500-1,000$ & $54.6 \%$ & $60 \%$ & $69.2 \%$ & $37.5 \%$ & 0 & $10 \%$ & $9.1 \%$ & \\
\hline \multicolumn{9}{|l|}{ blue collar low } \\
\hline $2,00 \|-5,000$ & & & & & $11.1 \%$ & $30 \%$ & $33.3 \%$ & \multirow{3}{*}{$\begin{array}{l}0 \\
85.7 \% \\
14.3 \%\end{array}$} \\
\hline $1,00 \|-2,000$ & & & & & $66.7 \%$ & $50 \%$ & $41.7 \%$ & \\
\hline $0,500-1,000$ & & & & & $22.2 \%$ & $20 \%$ & $25 \%$ & \\
\hline \multicolumn{9}{|l|}{ Others } \\
\hline $1,001-2,000$ & & & & & $42.9 \%$ & $33.3 \%$ & $58.3 \%$ & \multirow{2}{*}{$\begin{array}{l}28.6 \% \\
71.4 \%\end{array}$} \\
\hline $0,500-1,000$ & & & & & $57.1 \%$ & $66.7 \%$ & $41.7 \%$ & \\
\hline
\end{tabular}

Sources" Firm Survey (2002)

The above results are consistent with the OLS regression reported in Table 6.6 below, which indicates that the average wages are positively correlated with and more sensitive to attained/actual education. For instance, Table 6.6 below illustrates that the average wages are increasing in actual/attained education and experience (cf. Mincer, 1974) and therefore, is biased against less educated and experienced workers. These findings support our results from the firm survey, which indicate that wages are increasing in education and biased against low educated workers because the ratios of high skilled to low skilled wages, which can be interpreted as wages/skills premium, exceeds one. ${ }^{137}$ These results are consistent with the findings in the new growth literature, particularly skilled biased technical change theorems (cf. Aghion and Howitt, 1992; 1998; Acemoglu, 1998; Autor, Katz and Krueger, 1998). Our results that required education has no significant impact on wages are somewhat surprising and different from our expectation in view of the results of the overeducation literature (Hartog, 2000; Muysken et al. 2001; 2002a;b; 2003). We find that the positive correlations between actual education, experience and wages are particularly significant for large size firms, which may not be surprising since these firms have sufficient scope for a coherent wage policy (Muysken and Nour, 2005). This is also probably because large size firms may have more consistent recruitment strategies and high skill levels - share of high skilled workers in total employment - see Figure 6.1 above. These results imply that an

${ }^{137}$ From the firm survey (2002) we find that the proportion of high skilled wages/low skilled wages accounts for $7.5,6.9,8.1,6.3$ and 8.4 for all firms, chemical, metal, large and medium size firms respectively. 
increase in skill level/actual education and firm size leads to an improved relationship between actual education, experience and wages.

Table 6.6-Conrelation between wages (log) actual and required education and expertichce (2001)

\begin{tabular}{|c|c|c|c|c|c|c|}
\hline & & \multicolumn{3}{|c|}{$\begin{array}{c}\text { Coefficient } \\
\text { (t-value) }\end{array}$} & \multirow[t]{2}{*}{$\mathrm{R}^{2}$} & \multirow[t]{2}{*}{$\mathbb{N}^{138}$} \\
\hline Independent variable & Group of firms & $\begin{array}{l}\text { Actual } \\
\text { education }\end{array}$ & Experience & \multirow[t]{4}{*}{$\begin{array}{l}\text { Required } \\
\text { education }\end{array}$} & & \\
\hline \multicolumn{6}{|c|}{ Dependent variable: A werage wages (log) } & \\
\hline \multirow{7}{*}{$\begin{array}{l}\text { Average wages (log) } \\
\text { hight, medium and } \\
\text { low skilied }\end{array}$} & All firms & $\begin{array}{l}0.231 * * \\
(2.259\end{array}$ & & & 0.195 & 23 \\
\hline & Large & $\begin{array}{l}0.284 \% \\
(4545)\end{array}$ & & & 0.674 & 12 \\
\hline & All firms & & & \multirow{5}{*}{$\begin{array}{l}0.159 \\
(1.275)\end{array}$} & 0.119 & 14 \\
\hline & All fims & $\begin{array}{l}0.170^{\%} \\
(1.471)\end{array}$ & $\begin{array}{l}0.048 \\
(1.107) \\
\end{array}$ & & 0.242 & 23 \\
\hline & Large & $\begin{array}{l}0.193^{*} \\
(2.175)\end{array}$ & $\begin{array}{l}0.053^{*} \\
(1.374)\end{array}$ & & 0.730 & 13 \\
\hline & Chemical & $\begin{array}{l}0.243^{* *} \\
(2.001)\end{array}$ & $\begin{array}{l}0.05469 \\
(1.102)\end{array}$ & & 0.557 & 13 \\
\hline & All firms & $\begin{array}{l}0.192^{*} \\
(1.199) \\
\end{array}$ & $\begin{array}{l}0.011 \\
(0.172) \\
\end{array}$ & & 0.254 & 14 \\
\hline
\end{tabular}

Corretation is significant * at the 0.05 level (one-tailed) **at at the 0.01 level (one-tailed)

Therefore, our findings in this section corroborate the first part of the second hypothesis that an increase in skill levels and firm size leads to improved relationship between actual and required education and experience; and between actual education, experience and wages. In the next section we proceed to examine the second part of the second hypothesis that an increase in skill levels and firm size lead to improved relationships between skill, upskilling and technology (ICT). Finally, we test our third hypothesis on the relationship between technology (ICT) and input-output indicators at the micro/firm level.

\section{4 Skill, upskilling (ICT training) and technology (ICT) and input- output indicators}

Based on the above results, in this section we examine the other part of the second hypothesis that an increase in skill levels and firm size lead to improved relationships between skill, upskilling and technology (ICT) across firms. Before examining this hypothesis, it is useful to briefly show the variations in the use of technology (spending on ICT) and upskilling

\footnotetext{
138 For this regression we use relatively few observations, because some of the respondent firms were particularly reluctant to provide adequate quantitative data 10 measure skill indicators. For instance, there are few observations when combining attained education, experience and required education, particularly so for required education. As we explained in Chapter 4 above, we excluded some observations due to inconsistency or unreliability. One major problem is the varying response rate for different questions (to measure attained and required education, experience and wages) across frms. Moreover, the classification of frrms into chemical/metal, medium/large also divided the few observations between them and, therefore, allow for only a few observations for regression for each group independently.

139 The required education is not used as a variable in the upper half of Table 6.6 , becausc, we want to check the relation with respect to actual/attained education and experience independently and then compare the result when the required education is also included in the regression.
} 
$n$ 
CHAPTER 7

\title{
Challenging the serotonergic system in Parkinson's Disease patients
}

Effects on cognition, memory, motor performance and mood

\author{
Bart Scholtissen "Frans R.J. Verhey" , Jos J. Adam; Albert F.G. Leentjens" \\ alnstitute of Brain and Behaviour. Maastricht University, Maasuricht, The Netherlands \\ "Department of Psychiatry Maastrichi: University Hospital, Maastrich. The Netherlands \\ - Department of Movement Sciences, Mastricht University. Maastricht, the Netheriands
}

\section{Abstract}

Parkinson's disease (PD) is a neuropsychiatric disease which is not only characterized by motor symptoms, but also by cognitive and psychiatric symptoms. Multiple neurotransmitter systems are involved in the pathophysiology of the disease, including the serotonergic system. Although it is hypothesized that serotonin might have a wider influence than merely being responsible for psychiatric aspects of $\mathrm{PD}$, the exact role of the serotonergic system remains unclear. The aim of the present study was to investigate the influence of serotonin on cognition, memory and motor performance in PD. In a double blind, randomized, placebo controlled, crossover design, the effects of aspecific and specific serotonergic loading on the Visual Verbal Learning Task (VVLT), the Concept Shifting Task (CST), Profile of Mood State Questionnaire (POMS), the motor section of the Unified Parkinson's Disease Rating Scale (UPDRS, section 3), Simple Reaction Time Task (SRT), and Finger Precuing Task (FPT) were investigated in 21 PD patients, in early stages of their disease and 21 age, sex and education matched healthy volunteers. Results indicated that challenging the serotonergic system in a specific or aspecific way resulted in similar effects for both groups. No effects were observed regarding the cognitive tasks (VVLT, and CST). Results of the POMS indicated that, at baseline, patients showed a less positive score on all five subscales, than controls. Motor performance was affected by the challenge of the serotonergiic system. Both patients and controls react- 
ed comparable to the challenge, this was evident in motor slowing. The fact that patients and controls reacted in the same way to the different interventions may also be an indication that the serotonergic system is working similarly in both groups, and that the functional availability of serotonin is not affected in PD. Concluding, the effects of receptor specific and aspecific challenging of the serotonergic neurotransmitter system had similar effects in both PD patients and healthy control subjects. These findings indicate that the serotonergic neurotransmitter system is still operational in the tested population, and that serotonin, although involved in the pathophysiology, does not appear to play a direct role in motor performance, memory, and cognition, with the possible exception of hypokinesia in PD patients.

\section{Introduction}

The dopaminergic system is not the only system involved in the pathophysiology of Parkinson's disease (PD). Amongst others, the serotonergic system is also involved in this process. Degeneration of serotonergic neurons, decreased serotonin content, and alterations in the activities of various serotonin receptor subtypes, have all been demon-. strated in post-mortem studies, using neurochemical and autoradiographic techniques. [1-9]. The impact of this reduced serotonergic activity in PD, however "remains unclear.

From animal research it is known that serotonin reduces the activity of dopaminergic cells in the substantia nigra pars compacta and reduces striatal DA release $[10,11]$. Based on these observations, a compensatory role of reduction of serotonergic activity for the reduced striatal dopamine (DA) activity is hypothesized $|12,13|$. Moreover, serotonin activity influences several cognitive functions, such as memory consolidation and attention shifting |14-16].

The aim of the present study was to investigate the role of serotonin in cognitive and motor function in PD patients by temporarily elevating the level of avallable serotonin in the brain by administrating a Specific Serotonergic Reuptake Inhibitor (SSRI, citallopram) or $5-4 T_{\text {ya }}$ agonist (buspirone). Intake of both substances results in an acute elevation of central serotonergic availability $[17,18]$. The fact that we were interested in both the effects of a general increase of available serotonin and the receptor specific mediated increase of serotonin led to the choice for both abovementioned substances. Based on results from earlier investigations and theoretical grounds $[12,13,19]$, we hypothesized that this challenge would have the opposite effect of acute tryptophan depletion (ATD), thus resulting in a positive effect on cognitive performance. Furthermore since serotonergic levels of PD patients are already lower than the levels of the healthy control subjects, a greater improvement might be observed in patients. In addition, due to an increased inhibition of striatal dopamine release, as based on results 
from preclinical data, we expected a negative effect on aspects of motor performance in PD patients.

\section{Methods}

\section{Subjects}

Twenty one PD patients were included in the challenge study. They were recruited from the neurological outpatient departments of the Maastricht University Hospital, and the Atrium Medical Center Heerlen. All patients were diagnosed with M.Parkinson, according to the United Kingdom Parkinson"s Disease Society Brain Bank (UK PDS BB) criteria [20]. Participation in the study did not have any influence on the medical treatment patients were receiving. Patients who were diagnosed with any neurological disease other than M.Parkinson, or with any psychiatric disorder, including depression, as defined by the criteria of the Diagnostic and Statistical Manual (DSM IV) of the American Psychiatric Association (APA) [211, were excluded from participation in the study. The presence of any psychiatric disorder, notably major depressive disorder, was established in a psychiatric interview. Other exclusion criteria were the use of psychoactive medication, such as antidepressants and antipsychotics, the use of L-dopa, the dopamine agonist lisuride, selegeline, the abuse of alcohol or drugs, and cognitive disorders which was defined by a score on the Mini Mental State Examination (MMSE) of 23 or less. A prior personal or family history of depression was also considered a ground for exclusion.

Control subjects were recruited from an existing database of volunteer subjects of the Maastricht Aging Study (MAAS) [22], and individually matched with the PD patients concerning age, sex and education level. The same in- and exclusion criteria applied to the control subjects, with the exception of M.Parkinson. The study was approved by the Medical Ethics Committee of the Maastricht University Hospital. All subjects gave their written consent prior to participation and received a financial compensation for participating in the study.

\section{Design}

The study used a neuropsychiatric approach in which attention was given to several possible symptom areas, such as, motor, cognition, and mood. The study was conducted according to a double-blind, placebo controlled, randomized cross-over design. Intervention consisted of a placebo, a $40 \mathrm{mg}$ dose of citalopram, or a $30 \mathrm{mg}$ dose of buspirone. The intervention days were at least 7 days apart in order to rule out any carryover effects. 


\section{Intervention}

On the three test days subjects received a capsule at two time points ( 10 and 2 hours) after having completed the baseline tests in the morning. The capsules contained either a placebo mixture, $40 \mathrm{mg}$ of citalopram, or $30 \mathrm{mg}$ of buspirone. Since citalopram and buspirone are not absorbed equally fast by the body. we chose to give subjects a capsule at two time points within one day. Regardless of the content the substance would be maximally absorbed at $3 / 2$ hours after ingestion of the first capsule. For further clarification see figure 1 .

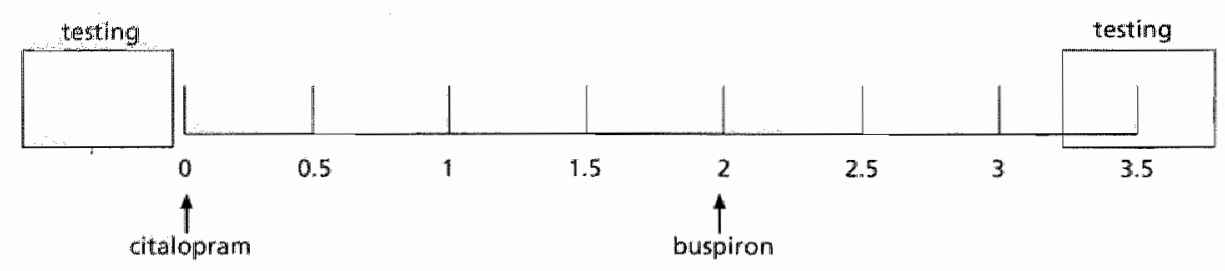

Figure 1. A schematic overview of the test day, numbers indicate time in hours.

\section{Procedure}

Subjects underwent a short physical examination, to rule out any potential physical exdusion criteria. Furthermore, the Hamillton Rating Scale for Depression (HAMD), and the Mini Mental Status Examination (MMSE) were obtained to characterize the population [23]. All patients were staged according to the Hoehn and Yahr staging system [24]. All subjects underwent a training session before the actual three test days to control for learning effects. On the test days subjects arrived at 9:00 a.m. Starting the day, a baseline measurement was obtained after which the subjects received the first capsule. Two hours after the intake of the first capsule participants took the second capsule. The measurements were performed again at the point of maximal challenge, which was at $31 / 2$ hours after intake of the first capsule. Subjects were free only to drink water, and eat a light lunch at noon. After finishing the afternoon test session, participants were able to return home.

\section{Outcome measures}

The design of the test battery used in the present experiment was similar to the one used in earlier experiments in our group (5choltissen, et al. I Neurol sci, accepted). The Visual Verbal Learning Task (VVLT) and the Concept Shifting Task (CST) were administered to 
investigate the effects of ATD on cognitive functioning, more specific: learning, memory consolidation and mental flexibility. In order to get an impression of the mood status of the subjects, the Profile of Moods 5tate (POMS) Questionnaire was administered (25). Furthermore, motor performance was examined using the Unified Parkinson's Disease Rating Scale (UPDRS) and two reaction time tasks, a simple Reaction Time task (SRT), and the Finger Precuing Task (FPT). All tasks were obtained at baseline and $3 / 2$ hours after intake of the first capsulle.

\section{Visual Verbal Learning Task}

The VVLT is an adapted version of the Auditory Verbal Learning Task [26]. Fifteen words are presented three times in the same sequence on a computer display. After each of the three trials subjects are asked to recall the words (immediate recall). Twenty-five minutes after the presentation of the first trial subjects are requested to recall as many words as possible (delayed recall). This is followed by a recognition task consisting of 30 words, of which 15 are previausly presented and 15 are new but comparable words. The subject has to respond to the words by answering yes or mo, indicating the recognition of the words (delayed recognition). Parallel versions were used at different time points.

Outcome variables were the maximum number of words recalled in either of the three immediate recall trials as a measure of immediate recall from short term memory, the number of correctly recalled words on the delayed recall as a measure of retrieval from long term memory, and the percentage of correctly recognized words on the recognition task as a measure of long term memory storage.

\section{Concept Shifting Task}

The CST [27] is derived from the Trail Making Test [28] and is used as a measure for cognitive speed, visuomotor tracking and cognitive flexibility (the ability to switch between two concepts, e.g. letters and numbers). The CST consists of five parts. On each sheet, 16 small circles are grouped in a larger circle. In the first three parts the circles contain

either numbers (part A), letters (part B), or a combination (part C), all appearing in a random order. The subjects are requested to cross out the items as fast as possible in ascending order in part $A$ and $B$, and in part $C$ continuously change between numbers and letters (e.g. 1-A, 2-B, 3-C, etc). In the following two part5 (01 and 02), the 16 empty circles have to be crossed out as fast as possible.

Outcome variables were the interference score, obtained by subtracting the mean time needed for parts $A$ and $B$ (in which the total time needed to perform both parts is composed of a cognitive component and a motor speed component) from the time needed for completing part $C$ (in which the total time needed is composed of the cognitive and motor component from parts $\mathrm{A}$ and $\mathrm{B}$, but also an interference component, 
since the subject has to change between wo strategiesi as a measure for cognitive flexibility, and part 02 as a motor score without cognitive interference.

\section{Proflle of Mood state questionnaire}

The POMS assesses five qualities of mood, being: depression, anger, fatigue, vigor, and tension. The POMS consists of 32 opposite word pairs divided by a visual analogue scale of $100 \mathrm{~mm}$. The POMS was used, instead of depression scales like the Hamilton depression rating scale (HAMD) or the Beck Depression Inventory (BDI), because of its capability to assess mood states at several time points during one day and sensitivity for detecting transient mood fluctuations $\{29,30\}$.

\section{Unified Parkinson's Disease Rating Scale}

The UPDRS $\mid 31 \|$ is a widely used dinicalscale rating several aspects of PD, among which activities of daily living, motor performance, and medication side effects. In the present study only part 3, the part measuring motor performance, was used.

\section{Simple Reaction Time Task}

The SRT (32) is used as a measure of speed of information processing. The subject has to react to a target stimulus which is preceded by a warning stimulus. The subject is instructed to focus his/her attention and eyes on an empty square (35.35 mm) on the computer screen, and press down a button in front of him using the dominant index finger. A warning cue (a flashing red square) is presented for $1000 \mathrm{~ms}$, and after a variable time interval the square turns green (the imperative or target stimulus) and the subject has to let go of the button (Reaction Time) and move to and touch the green square on the computer screen as fast as possible (Movement Time). The time intervals between of fset of the warning signal and onset of the target stimulus ranged from 100 to $3000 \mathrm{~ms}$ and were divided into a short (100-1000 ms) medium (1050-1500 ms) and a long (1550-3000 ms) interval. Reaction time, movement time, and the percentage of errors were used as dependent or outcome variables.

\section{Finger Precuing Task}

The finger precuing task is a four choice reaction time task (with the index and middle finger of both hands operating four linearly arrayed response keys), with a precue signal providing information about which fingers to use for responding. That is, the precue reduces the number of possible reactions from four to two by specifying two out of four possible target locations. Subjects have to respond to a single target stimulus, which appears either after a short or a long preparation interval $(500$ and 2000 ms respectively) initiated by the appearance of the precue signal, by pressing the corresponding button as fast as possible 133,34 . Four different precue signals can be dis- 
tinguished, indicating selective preparation of two fingers on one hand (hand-cued), the same two fingers on two hands (finger-cued), different finger on two hands (neither-cued), or no selective preparation (uncued). The uncued precue signal provides no advance information about the upcoming target stimulus and associated key press re. sponse so that no selective preparation is possible. This is a necessary control condition because it leaves the basic four-choice reaction task unaltered. Reaction time and the percentage of errors were used as outcome variables.

\section{Statistics}

This study used a placebo-controlled, double-blind, within- and between-subjects design. Dependent variables of the VVLT, CST, POMS, UPDRS, SRT, and FPT were analyzed using a General Linear Model (GLM) repeated measures design. Within subjects factors were "intervention" (placebo versus buspirone versus citalopram), "time" (morning versus afternoon), and "interval" (short, medium, and long regarding the SRT and short and long with respect to the FPT). "Disease" (PD versus control subjects) was entered as between subjects factor. Values of $p<0.05$ were considered significant.

\section{Results}

\section{Subjects}

21 Patients and 21 control subjects matched for sex, age, and education (13 men, and 8 women) were included in the study. Patients had a mean age of $61.2( \pm 9.1)$ years and a mean MMSE score of 27.7 ( \pm 1.5 ). These values did not differ statistically from the control subjects, who had a mean age of $61.3( \pm 9.9)$ and a mean MMSE of $28.5( \pm 1.4)$, $[t(40)=-0.032, p=0.974]$ regarding age and $[t(40)=-1.833, p=0.074]$ regarding MMSE score. Patients had a low mean Hamilton Depression Rating Scale (HAMD) score of 0.81 $( \pm 0.9$ ), indicating no clinically relevant signs of depression [30,35]. At the time of testing, one patient was treated with anticholinergic medication, two patients were treated with an NMDA-antagonist, four patients were on dopa-agonists, and 14 patients were treated with a combination of the medication described above. Patients had a median score of II regarding the Hoehn and Yahr scale (range II-III).

\section{Visual Verbal Learning Task}

At baseline no differences between both groups existed, regarding all outcome variables (immediate recall, delayed recall, and delayed recognition). Regarding all vari- 


\begin{tabular}{|c|c|c|c|}
\hline 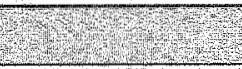 & Test Value & dif & P value \\
\hline \multicolumn{4}{|c|}{ Visual Verbal Learning Task } \\
\hline \multicolumn{4}{|c|}{ - effect of time of intervention } \\
\hline Immediate Recall & $F=39.45$ & 1,78 & $<0.0001$ \\
\hline Delayed Recall & $F=120.36$ & 1,78 & $<0.0001$ \\
\hline Delayed Recognition & $F=29.58$ & 1,78 & $<0.0001$ \\
\hline \multicolumn{4}{|c|}{ Proffile of Mood state questionnaire } \\
\hline \multicolumn{4}{|l|}{ - effect of interventiom } \\
\hline Depression. & $F=4.37$ & 2,78 & 0.016 \\
\hline Anger & $F=4.42$ & 2,78 & 0.015 \\
\hline fratigue & $F=5.89$ & 2,78 & 0.004 \\
\hline Tension & $F=3: 99$ & 2,78 & 0.022 \\
\hline \multicolumn{4}{|c|}{ Profile of Mood state Questionnaine } \\
\hline \multicolumn{4}{|c|}{ - effect of intervention by time } \\
\hline Depression & $F=7.59$ & 2,78 & 0.001 \\
\hline Aniger & $F=7.99$ & 2,78 & 0.001 \\
\hline Fatigue & $F=4.93$ & 2,78 & 0.01 \\
\hline Tension & $F=4.83$ & 2.78 & 0.0011 \\
\hline \multicolumn{4}{|c|}{ Profile of Mood State Questionnaire } \\
\hline \multicolumn{4}{|c|}{ - between subject effect } \\
\hline Depression & $F=8.67$ & 1,39 & 0.005 \\
\hline Anger & $F=4.6$ & 1,39 & 0.038 \\
\hline \multicolumn{4}{|c|}{ Simple Reaction Time Task } \\
\hline \multicolumn{4}{|c|}{ effect of intervention by time by disease } \\
\hline Reaction Time & $F=2.18$ & 4,152 & 0.073 \\
\hline Movement Time & $F=2.72$ & 4,152 & 0.032 \\
\hline \multicolumn{4}{|c|}{ Simple Reaction Time Task } \\
\hline \multicolumn{4}{|l|}{ - effect of time of day } \\
\hline Reaction Time & $F=19.24$ & 1,38 & 0.0001 \\
\hline Movement Tüme & $F=38.83$ & 1,38 & 0.0001 \\
\hline \multicolumn{4}{|l|}{ Finger Precuing Task } \\
\hline \multicolumn{4}{|c|}{ - effect of interval by oue by disease } \\
\hline Reaction Time & $F=3.56$ & 2,76 & 0.033 \\
\hline
\end{tabular}

Table 1. Overwew of effects on several tasks of the challenge experiment. 
ables no effects of intervention were observed, and intervention had similar effects in both patients and controls, using delta scores to correct for baseline performance. A significant effect of time of intervention (indicating an decreased performance at the second time point) was observed, using original data (no delta scores), $[F(1,78)=39.45$, $p<0.0001 ; F(1,78)=120.36, p<0.0001 ; F(1,78)=29.58$, $p<0.00011$, for immediate recall, delayed recall, and delayed recognition respectively, regardless of intervention (placebo, buspirone, or citalopram) (also see Table 1).

\section{Concept Shifting Task}

There were no main intervention effects and group differences regarding both the interference and motor score of the CST (using delta scores to correct for baseline performance). On baseline patients were slower than controls [t $(39)=3.37, p=0.002]$. Neither were any time of intervention effects observed.

\section{Profile of Mood State questionnaire}

Regarding the POMS subscale (depression, anger fatigue, vigor and tension), a significant difference between patients and control subjects existed on all subscales except vigor (all t's<0.05). Using delta scores to correct for baseline performance, a significant intervention effect was observed for subscales depression $[F(2,78)=4.37, p=0.016]$, an$\operatorname{ger}[F(2,78)=4,42, p=0.015]$, fatigue $[F(2,78)=5.89, p=0.004]$, and tension $[F(2,78)=3.99$, $p=0.022]$. Treatment with buspiron had a slight negative effect on the depression, anger, and fatigue subscales as compared to the placebo or citalopram intervention, whereas regarding the tension subscale intervention with citalopram had a positive effect, es. pecially for PD patients. Furthermore, a significant "intervention by time" interaction effect was observed for the same four variables $[F(2,78)=7.59, p=0.001 ; F(2.78)=7.99$, $p=0.001 ; F(2,78)=4.93, p=0.01 ; F(2,78)=4.83, p=0.011$, respectively], indicating that the aforementioned treatment effects where present at the last measurement of the respective test day. Although there was a significant between subjects effect in both the depression and anger subscales $[F(1,39) 8.67, p=0.005 ; F(1,39)=4.6, p=0.038$, respectively] indicating that PD patients showed lower scores than control subjects, no three-way interactions between intervention, time, and disease were observed (also see Table 1).

\section{Unified Parkinson's Disease Rating Scale}

Regarding the UPDRS section III (motor symptoms), no intervention effects were ob . served. Delta scores were used, in order to correct for baseline performance. Mean scores were 18.05 for the total section 111 at baseline, and 18.35 after the interverition. 


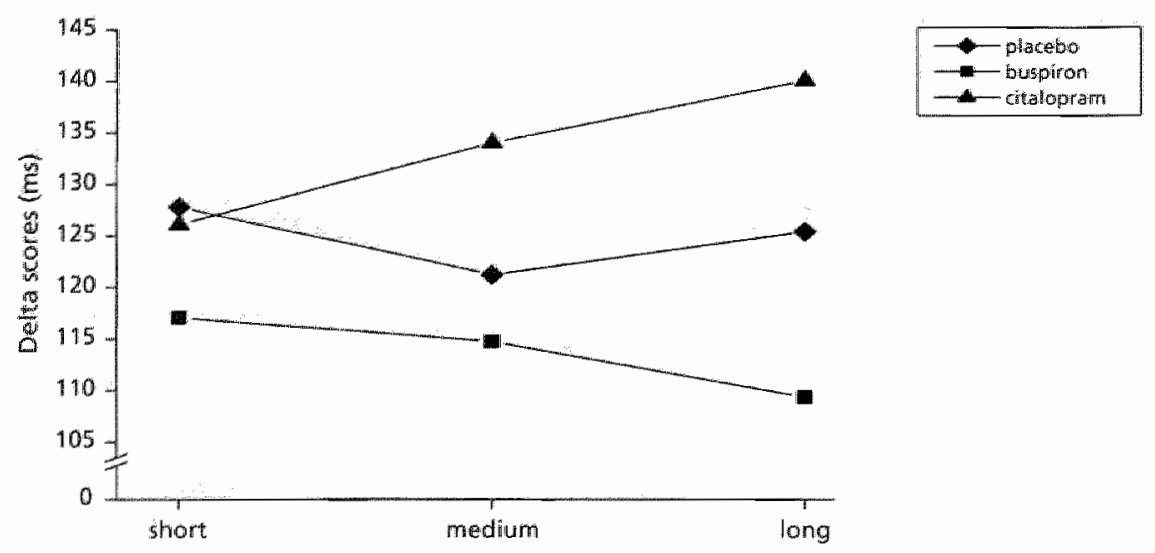

Figure 2. Effects of Buspiron and Citalopram versus Placebo in the SRT task, for PD patients relatiwe to control subjects over short, medium and long intervals, using deita movement time scores.

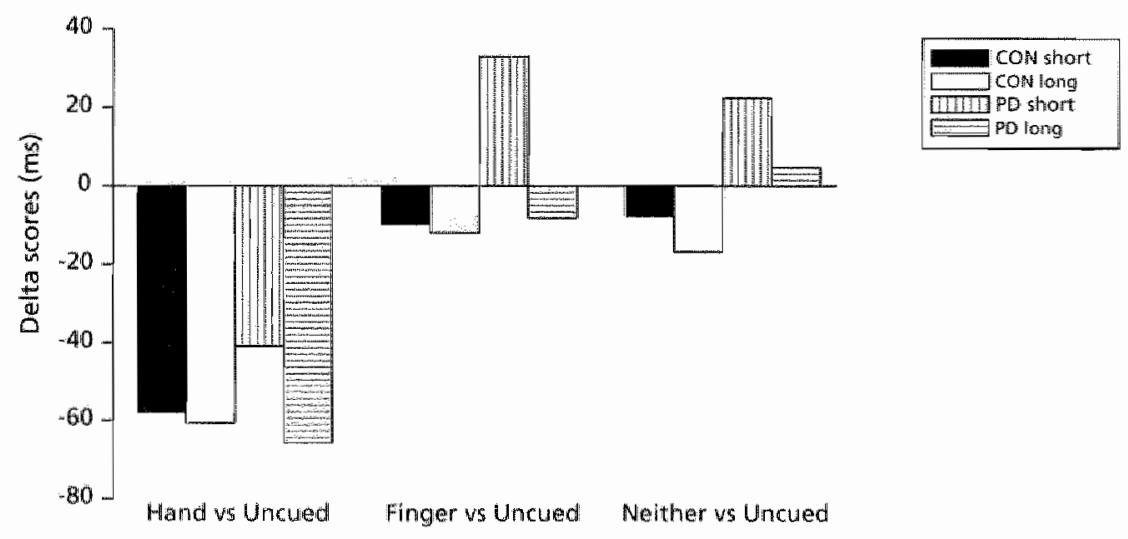

Figure 3. Precuing benefits (ms) for the hand cues, finger cues, and neither cues, relative to the uncued condition as a function of preparation interval (short and long) for PD patients and control sulljects. Negative scores indicate benefits (i.e. shorter RT in the cued than in the uncued condition). positive scores indicate disadvantages (i.e. longer RT in the cued than in the uncued condition). 


\section{Simple Reaction Time Task}

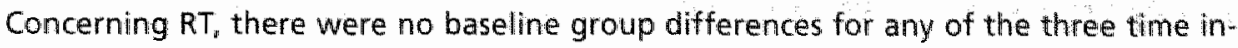
tervals $[t(38)=1.32, p=0.196 ; t(38)=1.44, p=0.158 ; t(38)=1.66, p=0.105$, short, medium, and long interval respectively]. Regarding MT, baseline differences (indicating slow. er movements of PD patients) were observed for all three time intervals $[t(38)=3.24$, $p=0.002 ; t(38)=3.17, p=0.003 ; t(38)=3.16, P=0.003$, respectively $]$. A near significant "intervention" by "interval "by "disease" interaction was observed for RT $[F(4,152)=2.18$, $p=0.073]$. For $M T$, this three-way interaction was significant $[F(4,152)=2.72, p=0.032]$. This three-way interaction indicates that relative to controls, PD patients showed a dif* ferent (slower) response in terms of MT to buspirone compared with placebo and citalopram intervention, especially for the long interval. Figure 2 illustrates this. Patients benefited more from buspirone than from citalopram with regard to the placebo condition. A general effect of time of day for both RT and MT $[F(1,38)=19.24, p=0.0001$; $F(1,38)=38.83, p=0.0001]$ was observed for both groups, indicating that performance at the second time point was always slower than at the first time point (also see Table 1). The percentage of errors was $1.03 \%$ for patients and $0.88 \%$ for control subjects. This difference was not significant $[t(39)=1.19, p=0.24$ n.s.].

\section{Finger Precuing Task}

Baseline differences, indicating slower performance of the PD patients relative to the control subjects, were observed for all but one (hand cued for the long interval) variable of the FPT (all t's<0.05). Furthermore, results showed a significant three-way interaction of "interval" by "cue" by "disease" $[F(2,76)=3.56, p=0.033]$, indicating a different response of patients and controls to the different cue conditions as a function of preparation interval (short and long) (also see Table 1). The difference between patients and controls was present for the short preparation interval, but not for the long interval. In particular, PD patients had great difficulty using short-duration precues $(500$ ms), as evidenced by a smaller preparation benefit in the hand-cued condition, and even a preparation cost in the finger and neither-cued conditions, that is, a longer RT in the finger-cued and neither-cued conditions than in the uncued condition (see Figure 3). In contrast, control subjects showed similar precuing benefits for the short and long preparation intervals. Also note that, overall, precuing benefits were much larger for the easy hand-cues than for the more difficult finger- and neither-cues. Overall, $\mathrm{PD}$ patients made significantly more errors than control subjects $4.49 \%$ and $1.52 \%$, respectively $[t(38)=5.3, p<0.0001]$. 


\section{Discussion}

The aim of the present study was to investigate the effects of aspecific and 5-HT1a receptor specific challenge of serotonin on memory processes, cognitive flexibility, motor performance, and mood in PD patients compared to sex, age, and education matched healthy controls. This is the first study to use this paradigm in patients suffering from PD. It is evident that a decrease of serotonergic activity exists in PD patients even in early stages of the disease, and that serotonin exerts an inhibiting effect on the release of dopamine $[10,11,36,37]$. However, functionally intervening with the system did not lead to a differential response between PD patients and control subjects. This implies that the activity of the serotonergic system is still high enough to react adequately on acute interventions in the system, and therefore does not appear to play a direct role in the symptomatology of the disease. Consequently, the decrease of serotonin levels does not serve as a compensation mechanism, but possibly constitutes a primary degenerative mechanism. In the case of mild PD patients, as those who were tested in the pres. ent experiment, this degeneration has not yet reached levels which make normal functioning impossible. If this would be the case, an acute intervention in the system would result in a direct change in serotonergic availability and thus also a direct change in the performance of the patient on the tests.

The observed effects of the present study only partially corroborated our expectations. Acute challenging of the serotonergic system appears to have no differential effects on memory, or cognitive speed and flexibility as measured by the VVLT and CST in both patients and healthy controls. Clear effects of time of day were observed over all intervention conditions, most likely indicating a nonspecific effect of fatigue. So, contrary to the results of ATD studies, in which a clear reduction of cognitive performance, more specifically an impairment in delayed recall and delayed recognition of long term memory (LTM) was observed (Scholtissen, et al, I Neurol sci, accepted, $\mid 38)$, cognitive functioning does not appear to be susceptible to acute increases in serotonergüc activity. This is in contrast with the findings of other researchers which suggest that administration of a serotonergic agent enhances long term memory 191 . This difference in results could possibly be explained by the age difference of both populations (approximately 60 years in the present study and approximately 40 years in the study of Harmer et al.). The population tested in the present study might have baseline age related memory impairments (present in both the controls as well as the PD patients). Challenging this aged system might not have the same beneficial effects as it has in other, younger, groups. The presence of mild forms of a neurodegenerative disease ${ }_{x}$ such as PD, appeared to be of no influence on this finding.

Since the serotonergic neurotransmitter system of PD patients appears to be more vuinerable, and this might reflect in the mood state of patients, the POMS was admin- 
istered. The results of the POMS also indicate that the serotonergic system is still working in a similar way in mild PD patients and healthy controls.

As was expected, the UPDRS scores did not change during each test day, nor over the different days. The UPDRS is not a very sensitive scale for detecting minor changes in motor performance. Motor performance, as measured by the SRT and FPT was, however, influenced by the acute challenge of the serotonergic system. The results of the SRT task demonstrate that both the RT as well as the MT component show a very consistent effect of time of day, for both patients and control subjects. Both patients and controls were slower at the second measurement compared to the first (baseline) measurement. Furthermore, the statistically significant three way interaction for MT between intervention (placebo, buspiron, citalopram), disease (PD vs. control), and intervall (short, medium, long) shows that PD patients react different on the interventions over the three time intervals. This means that, as can be seen in Figure $z_{\text {; }}$ the effects of buspirone are more beneficial for PD patients than the effects of citalopram with regard to control subjects. In other words, specific targeting of the 5-HTia receptor-5ubtype appears to be less disadvantageous for simple motor performance than elevating the general serotonergic levels in the brains of subjects, as is done by administering citalopram. Results of the FPT did not show these intervention specific effects. In this more complex task preparation benefits changed into disadvantages, especially for the PD patients, when the cue conditions became more difficult (the finger cue and neither cue conditions). This indicates that, apart from intervention, PD has an effect on the calpability of persons to benefit from cues in the execution of more complex motor tasks, especially when fast responses are demanded.

Regarding reaction time responding in general, the results described here, are complementary to the results of the ATD experiment. After all, an improvement in reaction time responding was found after ATD (Scholissen, et al., I Newrot Sci, accepted), while an impairment was observed after challenging the serotonergic system (as is visible in the positive deltal scores). This is interesting since it might indicate that serotonergic mechanisms are at least partially involved in motor processes in both normal subjects and PD patients. The fact that both groups basically reacted in a similar way, indicates that the serotonergic system in the patient group is still functioning the same way as in the control subjects. Since it is known from literature that even in relatively early cases of PD the serotonergic system shows signs of degeneration, the findings from the present study indicate that although the serotonergic system might not be functioning at its optimal level anymore, it is still able to react properly to functional interventions of the system.

It should be noted that there were some limitations to the present study. Patients who participated in the present study were mostly in stage II of the Hoehn and Yahr staging system, indicating that they were relatively early in the disease process, as is 
also demonstrated by the fact that none of the patients was using levodopa. Although including only drug-naive patients in a study would be preferred, it is barely possible to include large enough numbers of de-novo patients. Therefore, we decided not to exclude patients on medication rigorously, although the use of L-dopa, lisuride or selegeline, was an exclusion criterion. The inclusion of patients in earlier stages of their disease, as well as the use of medication might have introduced possible confounders in this study.

\section{Conclusion}

The present study showed that the effects of receptor specific and aspecific challenging of the serotonergic neurotransmitter system had similar effects in both PD patients and healthy control subjects. The observed effects were complementary with the results of previous ATD experiments. These findings indicate that the serotonergic neurotransmitter system is still operational in early PD patients, and that serotonin does not appear to play a specific and direct role in the pathophysiology of these patients.

\section{Acknowledgment}

The authors would like to thank P. Koehler for his help with recruiting patients.

\section{References}

(1) Scatton B, Javoy-Agid F. Rouquier L, Dubois B and Agid H, Reduction of cortical dopamine, noradrenaline, serotonin and their metabolites in Parkinson's disease, Brain Res, 1983275 (2) 321-328.

(2) Birkmayer Wand Riederer $\mathbb{P}_{n}$ Biochemische Veranderungen bei der Parkinson-Krankheit, In: Birkmayer $W$ and Riederer $P$ (Eds.), Die Parkinson-Krankheit; Biochemie, Klinik, Therapie, Wien Springe-Verlag, 1985, 29-59.

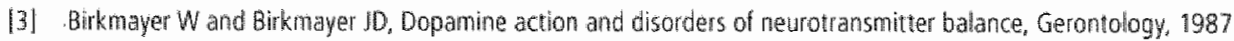
$33(3-4) 168-171$.

14] Chen $C P$, Alden JI, Bray $L$, Kingsbury AE, Francis PT and Foster OI, Post-synaptic 5-HTIA and 5-HT2A receptors are increased in Pakinson's disease neocortex, Ann N Y Acad Sci, 1998861288289.

151 Cheng AV, Ferier IN, Morris CM, labeen 5, Sahgal A, Mckeith IG, Edwardson JA, Perry RH and Perry EK, Cortical seratonin S2 receptor binding in Lewy body dementia. Alzheimer's and Parkinson's diseases, I Neurol Sci, $1991106(1) 50-55$

16] Maloteaux JM, Laterre EC, Laduron PM, Javoy-Agid F and Agid $Y$, Decrease of serotonin-S2 receptoss in temporal cortex of patients with Parkinson's disease and progressive supranudear palsy, Mow Disord, 19883 (3) 255 262.

[7] Perry EK, Perry RH, Candy JM, Fairbaim AF, Blessed G, Dick DI and Tomlinson BE, Cortical serotonin-\$2 recep- 
for binding abnomalities in patients with Alzhemet's disease: comparisons with Parkinson's diseasse, Neurosct Lete ${ } 988451(3) 353-357$.

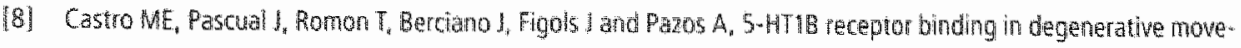
ment disorders, Brain Res, $1998790(1-2) 323-328$.

(9) Halliday GM, Bumbergs PC, Cotton RG, Blessing Why and Geffen L.8, Loss of brainstem serotonin- and substance P-containing neurons in Parkinson's disease, Brain Res, 1990510 (1) 104:107.

[10] Jacobs BL and Fornal CA, 5-HT and motor contol: a hypothesis, Teüds Neurosci, 199316 (9) 346.352.

(11) Ugedo L, Grenthoff I and Svensson TH, Ritanserin, a S-HT2 receptor antagonist, actiwates midbrain dopemine netrons by blocking serotonergic inhibition, Psychopharmacology (Ber). 198998 (1) 45-50.

[12] Mayeux R, Stern Y, Cote L and williams $\mathbb{B}$, Altered serotonin netabolism in depressed patients with parkinson's diseasie, Meurology. 198434 (5) 642-646.

(13) Mayeux $R$. The "serotonin hypothesis" for depression in Parkinson's disease, Adv Neurot, 199053 163-166.

[14] Buhot MC, Serotonin receptors in cognitive behaviors, Cur Op Neurobial, $19977243-254$.

[15] Buhot MC, Martin 5 and Segu L, Role of serotonin in memory impairment, Ann Med, 200032 (3) $210-221$.

116] Riedel W. Klaassen T. Deutz NE, wan Someren A and wan Praag HM, Tryptophan depletion in nomal volunteers produces selective impairment in memory consolidation. Psychopharmatolary (Berl), 1999 141 (4) 362-369.

[17] Peroutka 51, Selective interaction of nowel anxiolytics with 5-hydroxytlyptamine A receptors, 198520 (9) 971 . 979.

[18] Liu Y., Wikinson LS and Robbins TW, Effects of acute and chronic buspirone on impulsive choice and efflux of 5-HT and dopamine in hippocampus, nucleus accumbens and prefrontal cortex, $2004173(1-2) 175-185$.

[19] Harmer Cl, Bhagwagar Z, Cowen PJ and Goodwin GM, Acute administration of citalopram facilitates mernory consolidation in healthy volunteers, 2002163 (1) 106-110.

1201 Hughes AV, Daniel SE, Kifford L and Lees AI. Accuracy of clinical diagnosis of idiopathic Parkinson's disease: a clinico-pathological study of 100 cases. IN Neurol Neurosurg Psychiatry. 199255 (3) 181-184.

1211 DSM-IW, Diagnostic ans Statistical Manual of Mental Disorders, Washingtan, DC: American Psychiatric Assacia. tion, 1994.

[22] Jolles J, Houx PJ, Van Boxtel MPJ and Ponds R, 1995, The Maastricht Aging Study; determinants of cognitive ag" ing (Newropsychological Publishers, Maastricht).

23) Folstein MF, Folstein SE and MicHugh PR, "Mini-mental state". A practical method for grading the cognitive state of patients for the clinician, J Psychiatr Res, 197512 (3) 189-198.

124] Hoehn MM and Yahr MD, Parkinsonism: onset, progression and mortality, Neurology, $19671715 / 427 \times 442$.

(25) MC Nair DM, Lorr M and Droppotrman UF, Manual for the proflle of moods states, San Diego: Educational and Industrial Service, 1981.

[26] Rey A, Lexamen psychologique dans les cas de'encephalopathe traumatique (Psychological assersment in cases of traumatic brain ingury). Paris: Presses Universaires de france. 1964

127] Vink $M$ and Jolles 1 . A mew version of the Frail Maling Test as an information processing task, I clin Neuropsy, 19857162.

[28] Lezak MD, Neuropsychological Assessment. New York: Oxford Uniwersity Press, 1995.

[29] Beck AT, Ward CH, Mendelson M, MockJ and Erbaugh J. An Inwentory for Measuring Depression, Arch Gen Psythiatry, $19614561-571$.

[30] Hamilton Mi, A rating scale for depression, J Neurol Weurosurg Psy. 1960 $2356-62$.

311) Fahn 5 and Elton RL, Unified Parkinson's disease rating scale, New Jessey: McMlilan Health Care, 1987.

[32] Adam J], Paas FGWC, Buekers MJ, Wuyts 11, Spijkers WAC and Wallmeyer P, Perception-action coupling in choice reaction time lasks, Hum Mou Sci, $199615511-519$.

(33) Miler J. Discrete versus continuous stage madels of human information processing: in search of partial output. J Exp Psychol Hum Percept Perform, 1982812$\} 273-296$. 


\section{The manual tracking task for tremor assessment}

A new task for investigating manual tracking movement in PD

Bart Scholtissen", Ron F. Keulena, Frans R. \$. Verhey, Jos J. Adam" Albert F.G. Leentjens anstitute of Brain and Behawiour, Maastricht University.

Department of Movement Sciences, Mastrich University, Maasmichi, The Nethedands

"Department of Psychiatry. Maastricht University Hospital, Maastricht, The Netherlands

\section{Abstract}

Movement disturbances are core symptoms of Parkinson's Disease (PD). An uncomplicated task providing sensitive and detailed information about the severity of these symptoms would increase knowledge about and improve treatment options. The purpose of this study was to investigate (1) whether the Manual Tracking Task for Tremor Assessment (MTT-TA) is sensitive enough to detect tremor in early PD patients, compared to controls and whether simple manual tracking movements differ between these two groups, and (2) whether influencing the level of freely available serotonin in these subjects has any effects on the severity of tremor and manual tracking movements of both groups. As part of a larger test battery (described in chapter 5), the MTT-TA was used in a double blind, randomized " placebo controlled crossover design, in 15 PD patients and 15 control subjects. Both groups underwent the Acute Tryptophan Depletion (ATD) paradigm. Results indicated that no baseline differences existed between both groups. Furthermore, ATD had similar beneficial effects on the execution of the MTT-TA in both groups. In its present form, the MTT-TA lacks discriminant validity, but is very sensitive to change in performance of manual tracking movements. This implies that the MTT-TA is at this point not a suitable instrument for diagnostic purposes, but is suitable for the investigation of therapeutic effects of (pharmacologicall) interventions on tremor and manual tracking movements. Minor adjustments to the MTT-TA might improve the discriminant validity of the task. 


\section{Introduction}

Parkinson's disease (PD) is a neurodegenerative disease affecting approximately $1-2 \%$ of the general population. One of the most dominant features of PD is the disturbance of movement. Frequently, patients experience difficulties executing movements fluently. Movement disturbances are, however, not only caused by insufficient dopamine levels, other neurotransmitters are also involved in the pathophysiology of tremor, such as serotonin $\mid 1-5 !$. Since movement disturbances, such as tremor, are among the most invalidating aspects of PD, it is important to adequately and accurately detect and record the severity of these disturbances in a simple and patient friendly manner. Assessing the severity of movement disturbances (such as tremor) in a clinical setting is most often done by neurologists during consultations. A variety of methods for tremor assessment are available, such as the use of laser analog sensors, long term electromyography, actigraphy, and accelerometry [6-10]. However, these methods are mostly developed for application in an experimental (i.e., scientific) setting, and, more importantly, are often rather time consuming, which makes them less attractive for professionals in the clinical field. For the clinical situation a mare simple and practical device, providing the neurologist with information regarding the ability of the patient to perform simple manual tracking movements and the status of a possible tremor, would be ideal.

In the present study we investigated whether simple manual tracking movements and tremor were impaired in early PD patients relative to control subjects using a newly developed task, the Manual Tracking Task for Tremor Assessment (MTT-TA). The purpose of this study was to investigate (1) whether the MTT-TA is sensitive enough to detect tremor, and performance differences between early PD patients and contral subjects, and (2) whether reducing the level of freely available serotonin in these subjects improves tracking performance of both groups. We expected to find an improvement in movement execution after lowering the level of serotonin, since it is known from animal studies that serotonin inhibits the release of dopamine at the level of the strial* tum $|11|$.

\section{Methods}

\section{Subjects}

The present article describes data which are part of a larger data set in which 15 pathents and 15 control subjects matched for sex, age, and education were included (Scholtissen et al., I Neurol Sci accepted). The MTT-TA was also performed as a part of the challenge 
experiment described in chapter 7. Due to technical reasons, only data of the acute tryptophan depletion (ATD) experiment will be described here.

\section{Intervention}

The ATD experiment consisted of a placebo condition and a tryptophan depletion condition. Subjects were tested prior to the intervention, as a baseline measure, and $5 \mathrm{v} / 2$ hours after intake of either the placebo mixture or the tryptophan free mixture, when depletion of the available serotonin had reached a maximum (for an extensive descrip. tion see chapter 5 ).

\section{Procedure}

In the MTT-TA, patients are required to perform manual tracking movements on a touch-sensitive LCD computer screen. The subject is seated on a height-adjustable chair in front of a PL-400 V1.3-4 digitizing tablet (WACOM) that was equipped with an embedded LCD-screen (screen diagonal $=14 \mathrm{inch}$ ). The far end of the tablet was raised to an angle of 35 degrees, and the subjects recorded their responses with a handheld $\mathrm{PL}-400$ pen (WACOM) directly onto the screen (as if the subject is going to write a letter). Two blue circles, ( $10 \mathrm{~mm}$ ) were presented on an imaginary horizontal line on the screen with a distance of $225 \mathrm{~mm}$ in-between the two circles (see figure 1). The subjects were instructed to place the pen tip of the aforementioned pen on the left blue circle. Immediately after having contacted the left circle, a red rectangle appeared to the right from and directly adjacent to the left circle, and immediately commenced moving toward the right circle, at a constant speed of $4 \mathrm{~cm} / \mathrm{s}$. Upon arriving at the right circle, the moving rectangle changed directions and resumed moving in the opposite direction, toward the left circle. The trial ended 1.5 seconds after the moving rectangle had returned to its original position right from the left circle. The subject was instructed to immediateily start tracking the rectangle once it had started moving, by moving the pen on the screen as if he/she were drawing a line. The subject was explicitly encouraged to keep up with and maintain the speed that was dictated by the moving rectangle. Furthermore, subjects were instructed not to reverse movement prior to having contacted the right circle, and not to remove the pen from the screen until having returned at the left circle. By using a moving rectangle for dictating the speed of the subject's move. ments we sought to prevent subjects from adopting differential strategies (i.e. trad. ing speed for accuracy, or the other way round). As mentioned above, subjects were required to perform manual tracking movements at a constant, imposed speed and in a straight, horizontal line. Any disturbances in the execution of these manual tracking movements would likely be reflected in the variability in speed and/or vertical position 


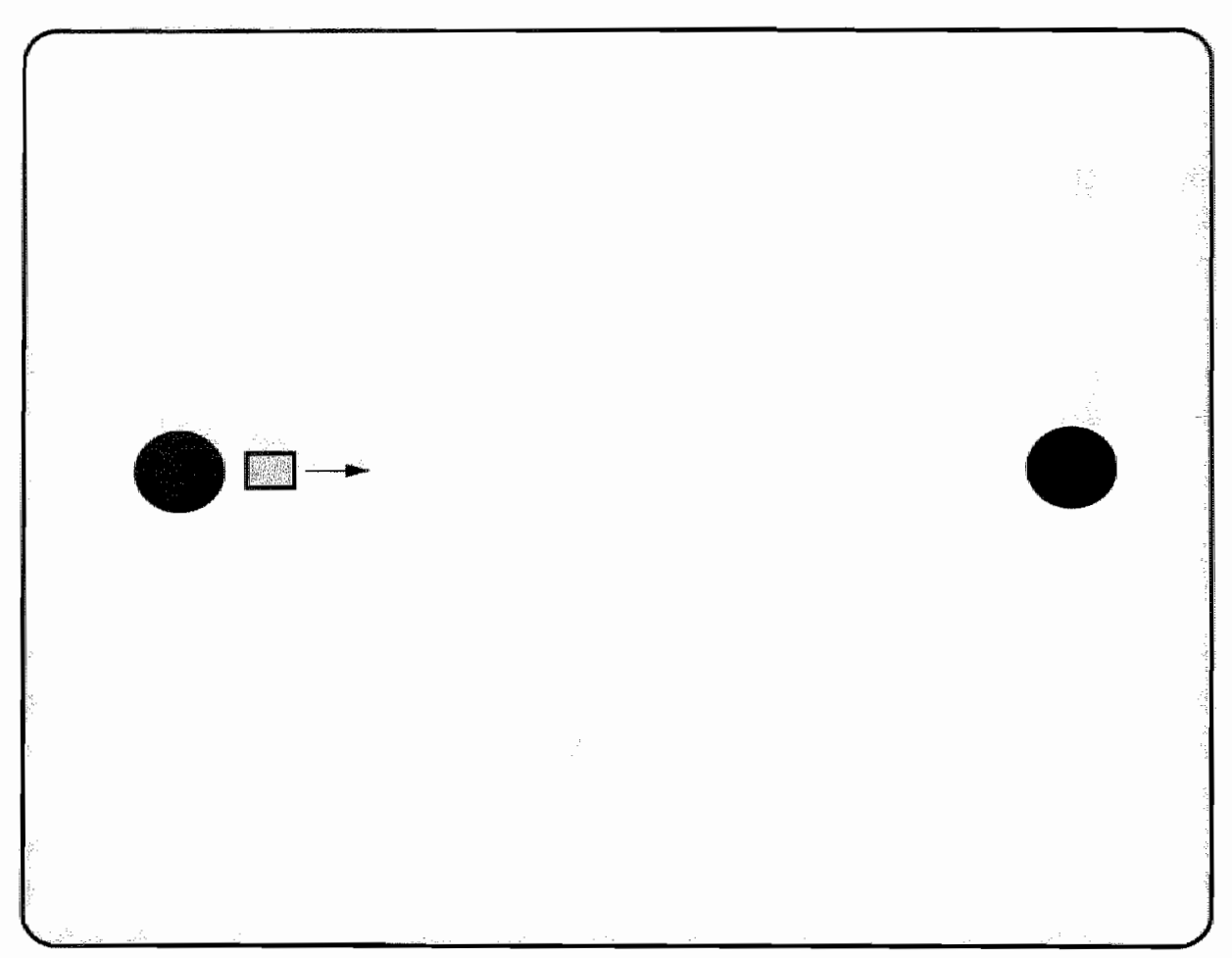

Figure 1. A schematic overwiew of the MTT.TA as displayed on a LCD screen. The arrow (not actually present in the test situation) next to the rectangle indlicaties the movement of the tectangle (at a constant speed of $4 \mathrm{~cm} / \mathrm{s}$ ).

of the movement. We therefore chose to focus on the standard deviations of tangential velocity (i.e. velocity irrespective of movement direction) and vertical pen tip position as dependent variables, as these are valid measures for irregularities in speed and vertical position. Furthermore, as we were primarily interested in the quality of the ongoing movement (rather than the initiation and termination of movement) we chose to restrict analyses to the central part of the movement trajectory, thus leaving out the initial and final $25 \mathrm{~mm}$ of each tracking movement (in either direction) in order to exclude possible confounding of initiation and cessation of the movement.

Administration of the MTT-TA consisted of five practice trials and ten actual test trials, the average of the 10 actual test trials being used for statistical analysis. The total time needed for the execution of the MTT-TA is approximately 5 minutes. The digitizing tablet was interfaced with an MS-DOS Pentium II computer equipped with a Matrox Millennium G400 DualHead Max graphical card. The OASIS 833 software package (KIKO Software, Doetinchem, The Netherlands) controlled stimulus presentation and 
recorded the spatial, temporal, and kinematic data. The temporal resolution was $5 \mathrm{~ms}_{\text {, }}$ and the spatial resolution was $0.5 \mathrm{~mm}$.

\section{Statistics}

Subjects ${ }^{*}$ performance on the MTT-TA was examined Using a General Linear Model (GLM) for repeated measures. Within-subject factor was intervention (placebo vs. ATD). The between-subject factor was disease (control vs. PD). Standard deviations (SD's) of tangential velocity $\left(V_{*}\right.$ in $\left.\mathrm{cm} / \mathrm{s}\right)$ and vertical position $\left(Y_{s}\right.$ in $\mathrm{cm}$ ) were calculated for both the rightward $(R)$ and the leftward $(L)$ portion of each trial. This resulted in the following dependent variables: SD-V-R, SD-Y $R$, SD-V-L, and SD- $Y-L$. These variables were chosen because of their relation with the clinical symptoms of tremor. $50-V$ provides information about the severity of the deviation of the tangential speed with which the line is drawn. The bigger the deviation in this speed, the larger the fluctuation in the execution of a (fluent) movement will be. This might also provide an indirect indication for the severity of tremor, since tremor has an effect on the degree in which a movement can be executed fluently. SD-Y provides information about the severity of the deviation in the vertical direction and reflects the change in amplitude of the tremor and movement. Separate Student's T-tests were performed for comparing baseline performance of patients and controls. For assessing the effect of the intervention, delta scores were used correcting for baseline performance. An alpha level of 0.05 was used to determine statistical significance.

Besides the abovementioned variables, other variables such as duration of the movements, and delay of pen position with regard to the moving rectangle were also analyzed. Since no effects were observed regarding these variables, they will not be discussed here.

\section{Results}

\section{Subjects}

Fifteen patients (nine men and six women; mean age $61.9 \pm 7.5$ years, median score of II on the Hoehn and Yahr scale (121) and fifteen sex-, age-, and education-matched controls (nine men and six women; mean age $60.8 \pm 8.8$ years) were included in this experiment. Registration of the MTT-TA failed in two patients and one control subject, due to software problems. The corresponding two control subjects and one patient were also excluded from statistical analysis, therefore, the statistical analyses were performed on the data of twelve patients and twelve controls. Instructions were well understood, 

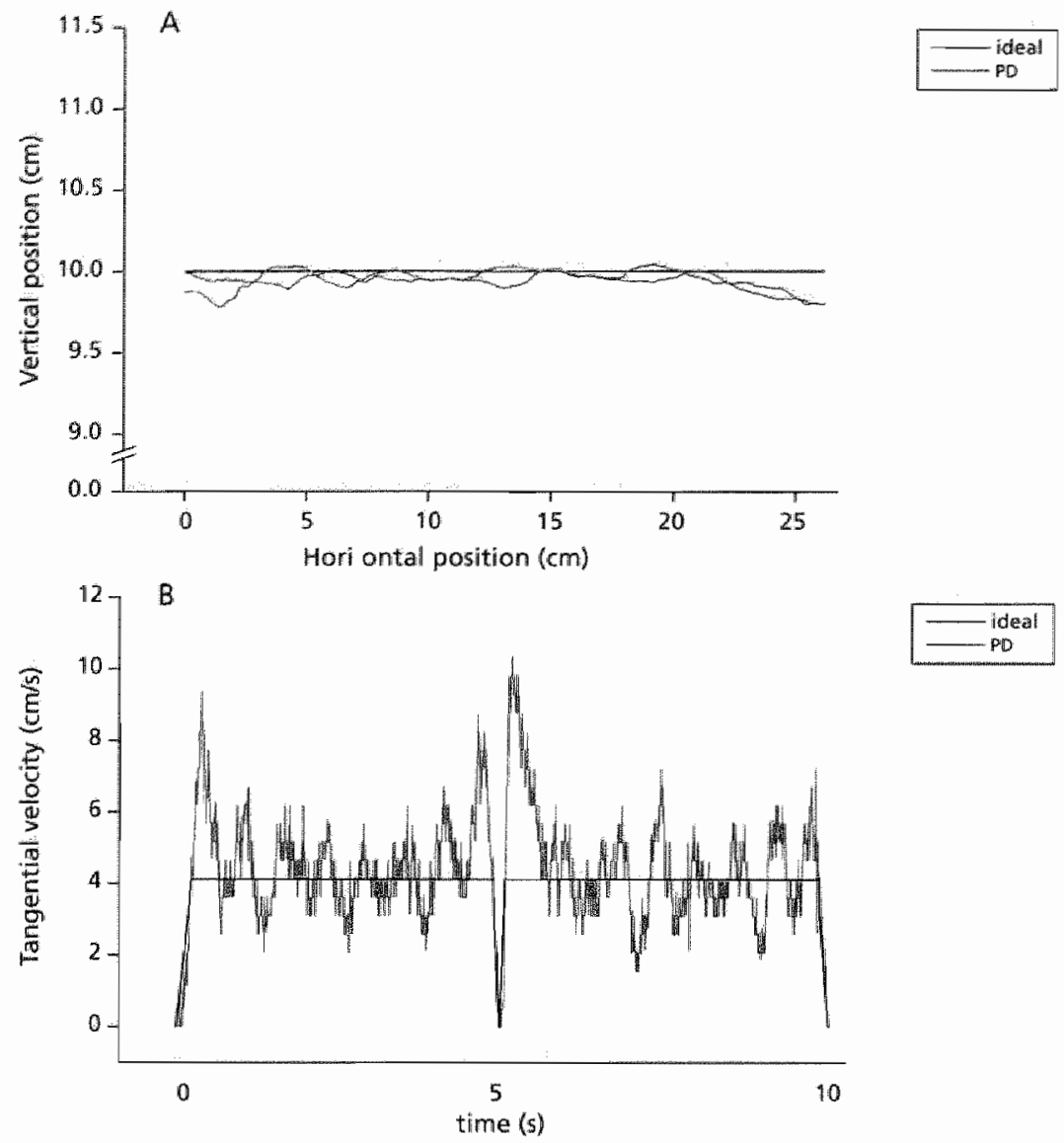

Figute 2. The upper panel (A) represents a sample line artually draw by a $\mathrm{FD}$ patient dung a random sample. The gray hod indicates the actual respanse of the patient, whereas the bick ine indicates what the ideal situation would look the (i.e a pefecty straight line). The bower pand (B) represents the tangential welocity of that particular sample ine. Note the difference between the actual speed igray line) and the ideal situatlon (black tine, indicating a mowement of constant speded).

\begin{tabular}{|c|c|c|c|}
\hline Sive & Test Nulue & ef & Prvalue \\
\hline SDVR & $F=128,53$ & 22 & $<0.0001$ \\
\hline SONR & $F=121,80$ & 22 & $<0.0001$ \\
\hline $50 \times 14$ & $F=10454$ & 22 & $<0,0000$ \\
\hline $50 \times 1$ & $F=64.39$ & 22 & $<0.0000$ \\
\hline
\end{tabular}

Table 1. Qverwew of interkertion effects of the MTT-TA. 
and all subjects were able to execute the required movements. Figure 2 provides an impression of an average line drawn by a PD patient.

\section{Baseline and intervention effects}

Results of the t-tests failed to illustrate a significant differences between PD patients and control subjects at baseline for any of the dependent variables (all $p^{\prime} s>0.1$ ). However, the ANOVAs with delta scores revealled a silgnificant main effect of intervention for all dependent variables (see Table 1). This intervention main effect indicated that the values of all dependent variables decreased as a result of acute tryptophan depletion. More specifically, a decrease in SD-V indicates that, the subjects' variation in movement velocity was smaller after ATD compared to the placebo condition. In other words, after ATD subjects experienced less difficulties in maintaining the speed as dictated by the moving rectangle. A decrease in SD-Y, on the other hand, implies that after ATD the subjects' variation in vertical dimension of the movement was smaller compared to the placebo condition. In other words, subjects experienced less difficulties drawing a straight line. Since both variables displayed a significant improvement after ATD, this means that ATD has a beneficial effect on movement execution, in that subjects were better able to follow a target moving at a constant speed. The effect of intervention did not interact with the direction of the movement $[F(1,22)=0.84, p=0.37$, ns. ] indicating that the magnitude of the intervention effect was similar in the rightward and the leftward portion of the tracking response. The lack of a significant intervention $x$ disease interaction suggests that there was no difference between PD patients and control subjects regarding the effect of ATD, thus implying that both groups responded to the pharmacological intervention in a similar fashion.

\section{Discussion}

This study was the first study using the ATD method and the MTT-TA in PD patients. The results of this pilot experiment suggest that acutely lowering serotonergic availability. using the ATD method, similarly improved the performance of both PD patients and matched controls on the MTT-TA, as measured by SD-V and SD-Y. This might indicate that, although it has been reported earlier that the serotonergic system may also be affected in patients with relatively mild PD [13], the serotonergic system in the PD population tested in this study is still functioning adequately and is sufficiently able to respond to acute changes in the serotonergic system. This option is strengthened by our observation that there were no performance differences in the execution of the MTTTA between PD patients and control subjects at baseline. These findings are in line with 
the antagonistic hypothesis of serotonin function in $\mathrm{PD}_{u}$ stating that the $5-\mathrm{HT}$ system facilitates motor output $[1,14]$. In literature, only one study has investigated the effects of lowered serotonergic functioning, using methysergide, a serotonergic antagonist [15]. In this study no improvement of motor function was observed, and findings were presented as evidence against a serotonergic mechanism in PD. However, this study had some methodological limitations.

Despite the lack of a main effect of disease, or a disease by intervention interaction in the present study, it appears that the MTT-TA is a sensitive task for assessing change in manual tracking performance, as depletion of serotonergic availability has proven to be reflected in a significant improvement of performance on this task. Both patients and controls displayed an improvement in performance after ATD. This indicates that after ATD all subjects experienced less difficulties executing the movement in a fluent manner, and thus deviated less from the ideal line, both in velocity and vertical position. ATD had a beneficial effect on the execution of movernent, in this case a manual tracking movement. This finding is in line with the results of earlier investigations, where we also observed an improvement in motor performance measured with reaction time tasks after ATD (Scholtissen ec all., submilted).

The fact that no baseline differences between PD patients and control subjects were observed might indicate that the present task is not sensitive enough to detect differences in performance of patients with mild PD compared to control subjects and, in its present form, thus lacks discriminant validity. This raises the question whether the task was not specific enough, or whether the population used for the assessment was not the right one. Looking at the tremor item of the Unified Parkinson"s Disease Rating Scale (UPDRS), five of the total of fifteen PD patients included in the study had a baseline tremor score of 2 or higher. This means that the average baseline tremor score of the total group is relatively low. Comparing a group of tremor dominant PD patients, with healthy control subjects, or a group of akinetic PD patients, might provide more information about the discriminant validity of the MTT-TA.

Furthermore ${ }_{i}$ it is possible that the speed of the moving rectangle was not optimal for the performance of this group of subjects. Slowing the imperative speed of the rectangle might have increased the sensitivity of the task. Another possibility might be that the use of a moving rectangle for dictating the velocity at which the movements were to be performed may have been responsible for the lack of a significant between groups effect. That is, there have been studies reporting that providing external cues

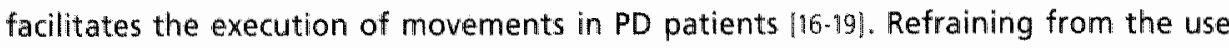
of a cuing rectangle, therefore, might be more suitable for providing a valid representation of the patients' ability to perform this task. More validation experiments of this task are needed before the task can be used in the clinical practice. 


\section{Conclusion}

No baseline differences in performance on the MTT-TA or differential effects between PD patients and control subjects after ATD were observed in the present study. This might indicate that compensatory processes are still able to counteract the degenerative processes in the brains of these mild PD patients. Therefore, administering this task to larger groups, as well as to patients who are in a more advanced stage of PD, and furthermore, adapting the task by removing the moving rectangle, might provide more information about simple manual tracking movements and tremor in PD patients. In its current state the MTT-TA lacks the required discriminant validity, but is sensitive to change, for instance after pharmacological interwentions. This means that the task is not suited for diagnosing disturbances in manual tracking and tremor in PD, but is suited for investigating the therapeutic effects of pharmacological interventions.

\section{References}

(1) Doder M, Rabiner EA, Turjanski N, Lees Al and Brooks DI, Tremor in Parkinson's disease and serotonergic dys. function: an 11C-WAY 100635 PET study, Neurology, 200360 (4) 604-605.

12] Edwards 16 and Anderson 1, 5ystematic rewew and guide to selection of selective serotonin reuptake inbibitors, Drugs, 199957 (4) $507-533$.

[3] Mehta $\mathrm{H}_{4}$ Saravanan $\mathrm{KS}$ and Mohanakumar $\mathrm{KP}$, Serotoan synthesis inhibition in olivo-cerebellar system attenuates harmaline-induced tremor in Swiss albino mice, Behav Brain Res, 2003 1.45 (1-2) 31-36.

[4] Leo Rs, Movement disturbances associated with the use of selective serotonin-reuptake inhibitors, Ann Pharmacother, $1998: 32712-714$.

15] Gerber PE and Lynd LD, Selective serotonin-reuptake inhbitor-induced movement disonders, Am Pharmacother. $199832692-698$

[6] Beuter $A$, de Geoffroy $A$ and Cordo P. The measurement of tremor using simple laser systems, 1 Newrosci Methods, $199453(1) 47-54$.

[7) Spieker 5, Boose A, Breit 5, and Dichgans J, Long-term measurememt of tremor, Mov Disard, 199813 Suppl 381 . 84.

18] van Someren EJ, Wonk BF, Thijssen WA, Speelman JD, Schuuman PR, Mirmiran M and Swablb DF, A new actigraph for long-term registration of the duration and intensity of tremor and movement, IEEE Trans Bioned Eng. $199845(3) 386-395$.

[9] Hoff Jl, van der Meer $V$ and van Hilten $\mathrm{II}$. Accuracy of objective ambulatory accelerometry in detecting motor complications in patients with Parkinson discase, Clin Neuropharmacol, 200427 (2) $53-57$.

[10] Thielgen I, Foerster F, Fuchs G, Hornig A and Fahrenberg J. Tremor in Parkinson's disease: 24- he monitoring with calibratted accelerometry, Electromyogr clin Neurophysiol, 2004 .44 (3) 137-146.

[111 Jacobs BL and Fornal CA, 5-HT and motor control: a thypothesis, Trends Neurosci, 199316 (9) 346-352

[12] Hoehn MM and Yahr MD, Parkinsonism: anset, progression and mortality, Neuralogy, 1967 17 (5) 427-442.

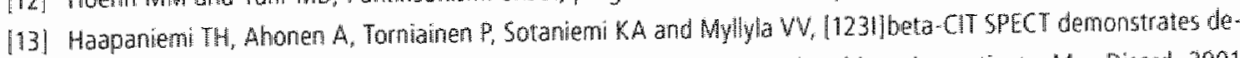
creased brain dopamine and serotonin transporter levels in untreated parkinsonian patients, Mov Disord, 2001 


\section{General discussion}

In this thesis, the aim was to bring two fields of research together. Starting out from the neuropsychiatric disease of Parkinson's disease (PD), the experiments described in this thesis attempt to bridge the gap between the clinical and preclinical fields of science. Attention was focused on the role of serotonin in PD. After providing an overview of the research done so far regarding functional interventions into the serotonergic neurotransmitter system in PD (chapter 2), the remaining part of this thesis focused on results from preclinical studies (chapters 3 and 4) and dinical studies. (chapters 5 to 8 ). In the preclinical part of this thesis, an animal model of PD (the 6-hydroxydopamine, or 6-OHDA model) was used to investigate the effects of several serotonergic and dopaminergic agents on behavioral performance and anatomical changes in the striatum of these animals. In the clinical part of this thesis, the effects of acutely intervening with the serotonergic neurotransmitter system on several symptom areas of patients suffering from Parkinson's disease were investigated. In this last chapter of the thesis, a brief review of the findings will be given and methodological limitations, and implications for future research will be discussed.

\section{Serotonin and Parkinson's disease: main findings of this thesis}

\section{Functional interventions of the serotonergic neurotransmitter system in PD patients}

Besides the well known motor symptoms, a myriad of other, mainly neuropsychiatric problems have been reported to occur in patients suffering from PD. These include, cognitive impailrments, apathy, dementia, depression, anxiety, psychosis, pain, sleep dysfunction, and behavioral dysfunction $\mid 1-14]$. It is not only dopamine that is causing the symptoms of the disease, but the degeneration of a number of other neurotransmitter systems may also play a part in the pathophysiology and symptomatology of the disease $[15,16]$. One of these other neurotransmitters is serotonin $[16 * 18]$. It is known 
that serotonergic activity is decreased in PD patients even patients with mild PD, and comorbid disorders in which it is assumed that serotonin is an important factor, such as for instance depression, zre observed more frequent in $\mathrm{PD}$ patients than in the general population [19-28]. The serotonergic hypothesis for depression in PD tries to give an explanation for these observations by stating that the serotomergic degeneration constitutes a compensatory response to the decreased striatal dopaminergic level, while at the same time this creates a risk factor for depression $129-31]$.

There are several ways of investigating the possible role of a neurotransmitter in a disease, for instance imaging studies, and postmortem neurochemical studies. Probably the most direct method of research is to functionally intervene with the neurotransmitter system in question. Chapter 2 is the result of a review of the existing dinical and preclinicall literature on data about functional interventions of the serotonergic system. This chapter provides evidence for the fact that preclinical and clinical studies appear to yield opposite results. While animal models appear to support the hypothesis that reduced serotonin levels form a compensatory response to the reduced dopamine levels, studies in humans do not appear to support this hypothesis.

Studies in humans with PD have clear advantages over animal models of PD which have serious shortcomings (for instance the different onset and recovery of the lesioned area and behavioral parameters) when investigating the pathophysiology of PD. Therefore, one of the conclusions of the review, and of this thesis in general, is that more effort should be invested in the use of the best available model of PD: patients suffering from PD. This can for instance be done by actively combining different fields of research, such as combining functional imaging research, with neuropsychological testing, and research investigating possible biological markers. Although preclinical research can also add more knowledge about basal mechanisms in PD, approaching PD from a clinical viewpoint is more likely to result in the discovery of clinically relevant information for better treatment of PD.

\section{Parkinson"s disease in animals, results from the 6-OHDA model}

PD is a disease that only occurs in one type of species, mankind. It has never been observed in any other species. From a clinical and scientific point of view animal madels are needed to further elucidate the characteristics and possible cure of a specific disease. It is, however, important to keep in mind that models are only a partial representation of the clinical reality. There are several ways of modeling $P D$ in animals $32-34$. One of the existing animal models for PD is the model we used for our studies, the partial bilateral striatal 6-OHDA model in the rat (for an overview see 1351 ). Results from chapter 3 indicate that after lesioning animals according to the 6-OHDA model, behavioral impairments in reaction time responding were observed. Also, a recovery of func- 
tion was observed in some (reaction time), but not all (movement time and premature responses), of the behavioral parameters at about two months after lesioning. Administration of dopaminergic ( $d$-amphetamine) and serotonergic agents (DOI and ketanserin) led to the conclusion that, in this model, serotonin and dopamine appear to be agonistically linked to each other. Administration of both the dopaminergic ( $d$-amphetamine) and serotonergic agent (DOI) resulted in an improvement of motor function, more specific reaction time. Chapter 4 describes in brief, that the observed behavioral recovery was paralleled by a recovery in Tyrosine Hydroxylase (TH) staining. This observation can be used to explain the behavioral recovery. The observed behavioral and immunohistochemical recovery implies that when using this model, a specific time frame (i.e. the first two months post lesion) has to be taken into account for behavioral testing، since the 6-OHDA model loses its usefulness for investigating PD symptoms after this period. Recovery is not new, although it is not widely cited in literature, there are a number of experiments describing (behavioral) recovery after 6-OHDA lesioning 13638). Therefore, it remains important to realize that besides the advantages of animal research, there also are serious limitations of animal models compared to the clinical situation (as mentioned earlier).

\section{Parkinson's disease in humans, results from the serotonergic depletion and challenge studies}

When interested in the exact role and function of a neurotransmitter, one of the most direct methods of investigation is functionally intervening with this system in the patient himself. This was done in two opposing ways: acutely depleting and challenging the serotonergic system. Chapter 5 provides data of the first study to date, in which the role of serotonin in cognition and psychomotor aspects in early stage PD patients was examined using this experimental approach. Results suggest that, after acute tryptophan depletion (ATD), cognitive and psychomotor performance (especially long term memory and reaction time performance) was equally affected in patients as in matched controls. The observations regarding the cognitive tasks (investigating short and long term memory and set shifting (apability) are mainly in line with results from earlier investigations, although some investigations failed to replicate the abovementioned findings $[39-45]$. The fact that similar results were found in both PD patients and control subjects, indicates that the serotonergic system still functions at a (near) normal level in these patients with mild PD, while it was expected to be more impaired since PD patients are known to possess a vulnerability for serotonergic dysfunction. in chapter 6 the serotonergic hypothesis for depression was tested. This hypothesis, originally formulated by Mayeux, is based on an overall reduction of serotonergic activity in PD $[29,30)$. This reduction can be seen as a functional compensation for the reduction 
in avallable striatal dopamine. Based on results from animal studies showing that serotonim inhibits dopamine release in the striatum, a reduction of available serotonin would result in less inhibition and lead to an increased availability of striatal dopamine $[46,47 \mid$. Furthermore, decreased serotonergic activity composes a biological risk factor for depression, and in PD depressive symptoms are more frequent than in the general population 121,24,31. After ATD, however, no changes in mood or cortisol response were observed. This speaks against the serotonergic hypothesis for depression. It appears that the vulnerability of PD patients for mood dysfunction is not directly mediated through the serotonergic system.

The data in Chapter 7 are based on the experiment following the ATD experiment. This experiment can be regarded as the opposite of the ATD experiment, and the same experimental setup was used. Here, the serotonergic system was acutely challenged, which resulted in comparable effects for both $P D$ patients and healthy control subjects. The observed effects were partially complementary with the results of our ATD experiment. After challenging the serotonergic system, cognitive performance (memory function and cognitive flexibility) was not altered, whereas motor performance (reaction time responding) was impaired. This impairment of motor performance was opposite to the improvement which was observed after ATD. These findings indicate that the serotonergic neurotransmitter system was still operational in patients with mild $\mathrm{PD}_{v}$ and that serotonin does not appear to play a specific and direct role in the pathophysiology of these patients. Bringing the results of the ATD and challenge studies together, it seems that a decrease of available serotonin leads to a partial improvement of motor performance, and a decrease in cognitive performance in PD patients in a similar way as in healthy control subjects. However, a decrease of serotonin did not lead to the expected impairment of mood, and therefore does not support the hypothesis of Mayeux and colleagues $(29,30)$. This hypothesis might not be completely valid, the first part of the hypothesis stating that a decrease in serotonin forms a compensational mechanism for the decreased striatal dopamine holds, but no evidence was found for the second part stating that there is an increased risk for depression. Therefore, attention should focus more on other neurotransmitters (for instance dopamine) regarding the regulam tion of mood symptoms. Increasing the serotonergic availability only partially led to the opposite results. Motor performance (reaction time responding) of PD patients was impaired but again in the same fashion as in healthy controls.

This finding also supports the observation that the serotonergic system in patients with mild PD is probably still functioning at an adequate level. Cognitive performance (memory function and cognitive flexibility) on the other hand, was not altered after increasing the serotonergic availability. This was also observed in other studies, although beneficial effects of serotonergic challenge on cognitive performance were also observed $|48,49|$. Motor performance can be directly influenced by interventions in the se- 
rotonergic system, but it also appears that serotonin does not play a direct role in the regulation of mood in the population tested in the experiments described in this thesis. All in all, although it is clear that a reduction of avallable serotonin results in memory impairments and improvements in motor performance, the opposite (increasing the availability of serotonin) does not lead to a complete opposite reaction, and thus the exact role of serotonin in PD still remains difficult to understand and needs further examination.

\section{Measuring simple manual tracking movement in PD patients, results from a new task}

The initiation and execution of movements is one of the core symptoms of PD and tremor is also a very frequent problem. For that reason, a simple task was developed to enable researchers and clinicians to investigate simple manual tracking movements and tremor in order to obtain a better insight in the severity and characteristics of this phenomenon.

Chapter 8 provides results of the first pillot study performed with this new task. Results indicated that in the population tested mainly mild cases of $\mathrm{PD}$, baseline performance between PD patients and control subjects was similar. This might imply that the disease process of the patients has not yet progressed far enough in order for the active. compensation mechanisms to fail, and for measurable differences to become manifest. But the fact remains that in its present form, the task lacks discriminant validity. Nevertheless, when acutely intervening with the serotonergic neurotransmitter system by means of ATD, beneficial intervention effects were visible. Using the standard deviation of the tangential movement as outcome variable, results showed that after ATD subjects were better able to follow a target moving at a constant speed and thus execute movements more fluently. The fact that, also after functionally intervening with the se. rotonergic system, no differences between patients and controls were visible can possibly be explained by the severity of the disease in the PD group, but can also be seen as support for earlier observations indicating that the serotonergic system appears to be functioning adequately in the tested population. Since the provided cue (the moving rectangle) might facilitate the movement execution for the patients, as it can be regarded as an external stimulus, refraining from the use of this stimulus might provide a more valid picture of patients" performance. Although removing the cue from the task also removes the option of controlling the speed with which the movement is executed.

Testing both versions of the task in a larger population, and perhaps also testing several groups of patients with different disease stages is likely to result in a more clear picture of the situation. 


\section{Methodological considerations}

Doing research almost automatically means using a limited group of people or animals in order to say something about the general population. However, it is almost impossible to create a small group that exactly copies the general population. This means that it is inevitable to encounter some limitations in each study. These are addressed before the implications of the results can be discussed.

\section{Preclinical Experiments}

The model we used for our investigations, the partial bilateral striatal 6-OHDA model, was developed out of dissent with other existing models. These other rat-models are not all suited for extensive behavioral testing, most models are unilateral, which limits the possibilities of behavioral testing. These unilateral models are, however, the most widely used models in testing novel forms of antiparkinsonian medicines, while they actually may not provide the best possible information (after all, rotating behavior of animals and the clinical situation of a PD patient are not really similar). It is important that a good model resembles the dinical situation (or specific aspects of it) as much as possible 1501. The major difference between animal PD models and the clinical situation is the fact that allmost al models (except for the model using rotenone) are of an acute nature, and when the lesion is not complete regenerative processes may occur $[35,51]$. These are probably the most difficult features to overcome when creating an animal PD model. Furthermore ${ }_{n}$ since PD is a disease which affects the human brain in a bilateral way, a bilateral animal model is preferred to an unilateral model. As it was the first time this model was used, it was not clear whether this model would be suitable. Results showed that the first two months after lesioning a clear behavioral deficit was present for all motor variables. Surprisingly, a behavioral recovery of function over time was observed. After anatomical and immunohistochemical analysis of the brains of the animals, results showed that a recovery of the lesioned striatal area was visible. This anatomical recovery can be used to explain the recovery of behavioral parameters, but it also indicates that recovery of the lesioned area is an important aspect which should be taken into account when using this animal model.

Taking this knowledge into account, it would perhaps have been better when all behavioral tests were performed in the first two months post lesion, or even use parallel groups to ensure that all substances were tested at approximately the same time after lesioning. The fact that this was not done in the experiment described in this thesis might have introduced a confounding factor into the experiments described in this thesis. However, not all behavioral parameters showed signs of recovery (reaction time recovered, whereas movement time, and premature responses did not show signs of re- 
covery), as is evident from the results of chapter 3. This possibly means that not all processes involved in the execution of movements are susceptible to the recovery of the lesioned area. This is an interesting observation which would have been overlooked if all behavioral tests would have been performed in the first wo months post lesion. However, at the time of the experiment we had no information regarding the possibility of behaviorall recovery in this model. Regarding future research using this 6-OHDA model, the use of several parallel groups to ensure that all substances are given at the same time after lesioning should be preferred over sequentially testing different sub. stances over a longer time period in one animal. This sequential method is widely used in animal research, partially for ethical reasons (reducing the number of animals used). However, this method might not always result in reliable results since underlying mechanisms might influence the outcome of those studies.

Concluding, when modeling a disease or aspects of this disease, it is of the highest importance to realize and consider exactly what stage and which specific aspects of a disease you're interested in. The model should resemble these characteristics as close as possible. When these criteria are met, it is permitted to draw conclusions about these characteristics. The fact remains that rats are no humans and (usually) vice versa, and therefore the interpretation of results from animal studies should be done keeping this observation in mind. In the end, successful symptomatic modeling in animals will depend on, and should benefit from, a continuous exchange of experience and information between clinical and preclinical investigators $\mid 50]$.

\section{Clinicall Experiments}

\section{Study population}

PD is a disease with a heterogeneous presentation. No two PD patients are identical. This makes it difficult to select homogenous experimental groups. Taking this into account and using the inclusion criteria as described in chapters $5 \& 7$, we tried to select the best possible homogenous groups for experiments described in this thesis. This. was, however, not allways a simple task. Patients who participated in the studies were recruited from the Neurological Outpatient Departments of the Maastricht University Hospital and the Atrium Medical Center Heerlen. These are the two largest hospitals in the region receiving patients from a large population. This does not automatically imply that the patients included in the experiments described in this thesis are a good representation of the general population in this region. Only including patients from these (academic) hospitals might have introduced a referral bias in the study [52]. The fact that the patients who were included in the described studies were all very motivat ed participants, was in contrast with the less motivated control subjects. The motivation of the patients can most likely be explained by their interest in gaining more knowl- 
edge about their disease and helping fellow PD patients in the future. Control subjects, unfortunately but understandably, did not have this commitment to the subject of the research. This might have influenced the results of the studies, in that the higher motivation of the patients may have led them to invest more effort and attention in the execution of the tasks, which might have led to better performance and results. Furthermore, since we decided to exclude patients who were one L-dopa medication, the included patients were mostly in early/mild stages of the disease. Patients were also excluded when they suffered from other neurological or psychiatric diseases. This means that we intentionally investigated a subpart of the general population. For research purposes this is more pragmatic, since for the experiments described in this thesis the choice was made to investigate early/mild patients with as little as possible comorbid problems (for instance depression). This does not mean that all patients were medication free. The use of antiparkinsonian medication might have introduced a confounding factor. Although it would ideally be better only to include medication free patients, this is nearly impossible to achieve since the vast majority of patients is immediately provided with medication after being diagnosed with PD. Then again, if it would have been possible only to include medication free patients in the studies of this thesis, this would also have led to a decrease of the external validity of the study, since medication free patients are generally relatively healthy, younger, perhaps more motivated subjects, and represent only a minor part of the general PD population.

All in all, a number of possible confounding factors might have been of influence on the results described in this thesis. We deliberately chose to investigate the effects of serotonergic intervention in a group of PD patients in early stages of the disease. A number of other possible comorbid disorders such as dementia, depression, and anxiety, which without doubt exert their influence on the PD itself, were not present in the tested population but served as a basis for exclusion. The fact that only patients in early stages of the disease without comorbid disorders were included in the studies described in this thesis, does not mean that only young patients were included in the studies described here. We did not use an age limit for the inclusion of patients, and although the mean age was 61 , the oldest patients included in the study were 75 years of age at the time of testing. Extrapolation of the results of this thesis to the general population of PD patients should be done keeping the aforementioned in mind. But this applies to clinical research in general, and not just for the experiments described in this thesis. Therefore, the results of this thesis certainly are a positive addition to the existing knowledge about PD.

\section{Diagnostic criteria}

The patients included in the described studies were all diagnosed with PD before they entered the study. All patients fulfilled the operational criteria of the United Kingdom 
Parkinson's Disease Brain Bank (UK-PDS-BB) |53]. We did not perform an additional diagnostic assessment with, for instance, IBZM or B-CIT single positron emission tomography (SPECT) in order to verify the diagnosis of the attending physician. Therefore, it is theoretically possible that the physician could have misdiagnosed a patient. This is, however, very unlikely, since the clinical items of the UK-PDS-BB have a diagnostic accuracy of $76 \%$ in comparison with postmortem histological confirmation of the diagnosis $\mathrm{PD}[53]$.

\section{Repeated Testing}

Participants of the studies were tested repeatedly, the ATD experiment consisted of two and the challenge study of three test days. This could have led to a learning effect, which might have interfered with the outcomes of the tests. In order to cantrol for this learning effect, in all studies the participants received extensive information and practice trials before the actual test days. We furthermore used a double blind, randomized order experimental setup and parallel versions of tests (when available), which also decreased the risk of confounding learning effects.

\section{Conceptual considerations}

For many years PD was treated as a pure motor disease. James Parkinson's own description of the disease, which would only be named after him by the French neurologist Charcot in 1876, was: "Involuntary tremulous motion, with lessened muscular power, in parts not in action and even when supported; with a propensity to bend the trunk forwards, and to pass from a walking to a running pace: the senses and intellects being uninjured" [54]. This last sentence of his description also indicates that he thought of it as a pure motor disease. Meanwhile, it is obvious that PD is not simply a motor disease, but much more a neuropsychiatric disease in which the "senses and intellects" are more often impaired than in the general population $[21,27]$. Not only degeneration of the dopaminergic system, but also degeneration of several other neurotransmitter systems such as the cholinergic, noradrenergic and also the serotonergic system play an important part in the pathophysiology and symptomatology of the disease 145-181. The fact that several comorbid problems (especially depression) are frequently present in PD, may have consequences for the nosological concept of PD. Many symptoms of depression resemble symptoms present in PD patients, and thus PD symptoms might be mistaken for depressive symptoms and vice versa. It is important that both clinicians as well as researchers are aware of the overlap in symptoms between PD, depression, and cognitive impairment and the interplay between several neurotransmitter systems. PD is not a disease caused simply by dopaminergic degeneration, but is the result of the 
complex interplay and degeneration of multiple neurotransmitter systems. A broad and multidisciplinary approach is important when investigating and treating this disease, since symptoms are present in multiple domains.

\section{Clinical implications}

The results described in this thesis may have a number of implications for clinical practice. The fact that PD is more than just a motor disease, indicates that a multidisciplinary approach in dealing with this disease is needed [55]. This implies that, ideally, PD patients should not onily be assessed by a neurologist, but also warrant a neuropsychological and psychiatric assessment. A multidisciplinary Movement Disorders Clinic (MDC) provides an ideal framework for this approach. In a multidisciplinary MDC PD patients are assessed by a team existing of a neurologist, neuropsychologist and a psychiatrist, whose test are all specifically aimed at PD. Each individual patient is consecutively seen by the neurologist, neuropsychologist, and psychiatrist. The assessment and treatment of the patients should be performed according to a previously framed protocol. In this way, not only movement problems are examined, but mood state and cognition are also routinely assessed. This way no time is lost with unneeded tests, and results from the various lines of work can immediately be integrated. The benefit for the patient lies in the integral assessment which can result in less under diagnosis of depression and cognitive decline, certainly since cognitive decline can be treated in PD patients using acetylcholine inhibitors [56], better treatment of various symptoms and better psycho-education of both the patient and his caregivers. The benefit to the patient may not be that obvious. Therefore, the patient and his caregivers must receive clear information about the benefits of a multidisciplinary approach, since it might not seem logical to the patient that he is also seen by a neuropsychologist and psychiatrist when his primary complaint is for instance tremor or rigidity. Apart from the clinical possibilities of this approach to the neuropsychiatric disease of Parkinson, this approach also provides an ideal basis for scientific research of several aspects of PD.

\section{5-HT and Parkinson's disease}

Although evidence exists that a decrease of serotonergic levels is present even in patients with mild PD 231 , the functional consequences of these alterations are limited. In motor symptoms, cognition, and mood, the acute interventions in the serotonergic system described in this thesis resulted in similar effects in PD patients and controls. This can be regarded as evidence against the hypothesis stating that a decrease in serotonin levels in PD patients can be regarded as a compensatory mechanism for the decreased 
dopaminergic levels as well as evidence against the serotonergic hypothesis of depression in PD. The fact that the acute interventions described in this thesis resulted in similar effects in PD patients compared to matched controls implies that the serotonergic system in these mild patients is still capable of functioning in a similar way as it does in the control subjects. This means that although it is known that the serotonergic system also degenerates in PD, the degeneration in patients with mild PD has not yet reached a level influencing normal functioning (like with the dopaminergic system). This implies that due to the exclusion criteria that we used in our studlies, a group of patients was selected that did not demonstrate the hypothesized serotonergic vulnerability that might be present in a group of depressed PD patients.

The clinical implications of the preclinical experiments with animals are not that straightforward. The results from these experiments are opposite to the clinical situation, since elevating serotonergic levels in animals resulted in an improvement of motor functioning, while in humans this led to an impairment of motor functioning. Although very interesting, this once more shows that results from preclinical experiments should not be extrapolated to the human situation without consideration. Though preclinical research has shown to be invaluable for gaining basic knowledge about several biological processes, it is important to remember that comparisons are made between different species of mammals (rodents, primates, and humans). Even though they share several characteristics, and the brains are comparable in several functions, a one on one comparison remains difficult. Therefore, as mentioned before, more effort should be invested in performing research in the best available model: i.e. PD patients themselves. This might result in better treatment and possibly earlier detections of all PD symptoms.

\section{Where to go from here?}

In order to further optimize the treatment of PD (or even develop a cure), that is not based on restoring the function of just one neurotransmitter but rather adhering a 'multi-transmitter approach', it is important to gain more knowledge about the role and function of all neurotransmitter systems involved in the pathophysiology of PD, not only dopamine and serotonin. Since no neurotransmitter system operates in an isolated fashion, and the manifestation of a specific symptom will most likely not be caused by an alteration in the functioning of one specific neurotransmitter, interaction between different systems must always be taken into account when investigating aspects of PD (and for that matter, any other neurological or psychiatric disease) [57,58]. Recent literature, for instance, showed that besides serotonin, other neurotransmitters (in this case dopamine) might play an important role in the pathophysiology of depression 1591 . 
To achieve the abovementioned goal, more research is needed. Since PD is a heterogeneous disease it is important to investigate different subgroups of patients. So not only investigate and compare mild and severe groups, but also divide the population according to other criteria. These criteria can include medication use, since different types of medication, and the period of use, might have aspecific influence on PD symptoms. It is also reported that the placebo effect in PD patients is large, and that placebo medication even is capable of activating dopamine production of neurons which were considered to be degenerated $|60-62|$. Another potentially interesting criterion is age of onset of the disease. Young PD patients may display other disease characteristics compared to older PD patients. Furthermore, motor symptomatology, tremor dominant type vs. akinetic rigid type is an interesting criterion since it is hypothesized that akinetic rigid type PD patients have a higher risk for developing a depression [63]. Forming groups based on psychiatric and neuropsychological comorbidity (for instance depression, apathy, and mild cognitive impairment), may also provide new and useful insights into the disease process. Changes in the functioning of different neurotransmitters which result in the abovementioned psychiatric and neuropsychological symptoms can also have a specific influence on PD symptomatology.

This asks for large scale investigations in which the multidisciplinary MDC can play a central role. Combining functional imaging (fMRI) research with psychological and pharmacological research will also give more insight in the relation between neural processes and the behavioral symptomatology. This stresses the need for a longitudinal design. This method provides the opportunity to follow a group of patients for several years, and may provide very useful information about the disease process and its effects on both motor and cognitive functioning of the patient. The multidisciplinary MDC forms an ideal basis for this research. Merging the efforts of several multidisciplinary MDCs, may even lead to a population based approach, resulting in the generation of data and knowledge about various subpopulations of PD patients. Structurally testing patients on a regular and in particular, longitudinal basis might result in the discovery of new biological markers of the disease. With the possible discovery of new biological markers, it might even become possible to identify more risk factors of PD that eventually may add to prevention or a cure for this disease.

Finally, the aims of this thesis: bridging the gap between the clinical and preclinical research fields, and investigating the role of serotonin in PD were not always within reach. It is clear that both the clinical and preclinical fields of research have contributed a large amount of knowledge about the interplay between serotonin and PD. However, results from both fields cannot be compared with each other without caution. As described in this thesis, although the investigated mechanisms share similarities, the results appear to be at least partially incongruous with each other. Therefore, it is important to create and maintain the best passible form of continuous exchange of experi- 
ence and information between clinical and predinical investigators in order to produce valid and meaningful research [50]. Regarding the role of serotonin in $\mathrm{PD}$, the following can be said. Although there is evidence that serotonin is involved in the pathophysiology of PD, the results of the studies described in this thesis indicate that in patients suf. fering from a mill form of PD the serotonergic system still operates in a similar fashion as in healthy subjects. This finding stresses the importance of longitudinal studies, so that different stages of PD can be compared within and between patients, and more information about the role of neurotransmitters over these different disease stages be comes available. Adhering to a multidisciplinary, multi-transmitter approach seems to be the best way of investigating PD.

\section{References}

(1) Ford B, Pain in Parkinson's disease, Clin Neurosci, 19985 (2) 63.72.

12) Friedman JH, Behavioral dysfunction in Parkinson's disease, Clin Neurasci, 19985 (2) 87-93.

13) Trenkwalder C, Sleep dysfunction in Parkinson's disease, Clin Neurosci, 1998 5 (2) 107-114.

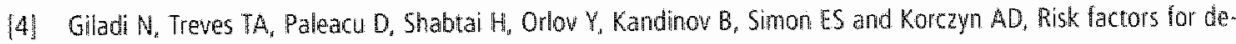
mentia, depression and psychosis in long-standing Parkinson's disease, INeural Transm, 2000107 (1) 59.71.

15] Aarsland D, Larsen JP, Lim NG, Janwin $C$, Karlsen $K$, Tandberg $E$ and Cumnings $U$, Range of neuropsychiatric disturbances in patients with Parkinson's disease, I Neurol Neurosurg Psychiatry, 199967 (4) 492-496.

16] Azuma T, Cruz RF, Bayles KA, Tomoeda CK and Montgomery EB, Jr. A longitudinal study of neuropsycholagical change in individuals, with Parkinson's disease, Int J Geriatr Psychiatry, 200318 (11) $1043-1049$.

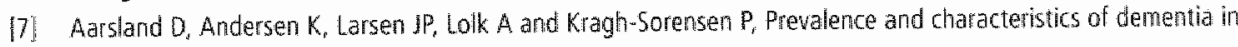
Parkinson disease: an 8-year prospective study, Arch Neurol, 200360 (3) 387-392.

[8] Schrag $A_{4}$ Psychiatric aspects, of Parkinson"s disease - an update, J Neurol, 2004251 (7) 795-804.

19) Enre M. Dementia in Parkinson's, disease: cause and treatment, Curr Opin Neurol, 200417 (4) 399-404,

(10) Hu MT, White S), Chaudhuri KR, Morris RG. Bydder GM and Brooks DJ, Contelating rates of cerebral atrophy in Parkinson's disease with measures of cognitwe decline, INeural Transm, 2001 108 15) 571.580.

[11] Dubois B and Pillon B, Cogmitive deficits in Parkinson's disease, J Neurol, 1997244 (1) 2 -8.

[12] Bruck A, Kurki T, Kaasinery Wathlberg T and Rinne 10. Hippocampal and prefrontal atrophy in pattents with early non-demented Parkinson's disease is related to cognitive impaiment, I Neurol Neurosurg Psychiatry, 2004 $75 \$ 10) \$ 467-1469$.

113) Mindham RH and Hughes TA, Cognitive Impaiment in Parkinson's Disease, Int Rev Pisychiatry, 200012281 . 289.

[14] Taylor AE and Saint-Cy AA, The neuropsycholagy of Parkinson's disease, Brain Cogn, 199528 (3) 281.296.

[15] Kisf 5]. Biochemistry of Parkinson's Disease: Is a Brain Serotonergic Deficiency a Characteristic of Idiopathic Parkinson's Disease. In: Gordin A, Kaakkola S and Teravainen H (Eds.), Parkinson's Disease: Advances in Neu. rology, vol 91, Philadelphia: Lippincot Williams \& Wikins; 2003.

[16] Jellinger $K$. Overview of morphological changes in Parkinson's, disease, Adv Neurol, 1986451.18.

117) Halliday GM, Li YW, Blumbergs PC, Joh TH, Cotton RG, Howe PR, Blersing WW and Geffen LB. Neuropathology of inmunohistochemically identified brainstern neuroms in Parkinson's disease, $199027(4) 373-385$.

[18] Scatton B, Javoy-Agid F, Rouquler L, Dubois B and Agid Y, Reduction of cortical dopamine, noradrenaline, sero- 


\section{Serotonit and Parkinsan I CHAPTEP 9}

tonin and their metabolites in Parkinson's disease, Brain Res, 1983275 (21321-328.

191 Bum D, Depression in Parkinson's disease, Eur I Meurol, 20029 Suppl 344.54.

201 Chen CP. Alder IT, Bray L. Kingshury AE. Francis PT and Foster Of, Post-smaptic 5HTIA and 5HT2A receptors are increased in Pankinson's disease neocorter. Ann N Y Acad So, 1998 B61 288-289.

(21) Cummings t, Depression and Parkinson's disease: a review, Am f Psychiazry, 1992 149(4) 443-454.

[22] Cummings $L$ and Masterman DL, Depression in patients with Parkinson's disease, Int I Geriatr Psychiatry 1999 $14(9) 7117118$

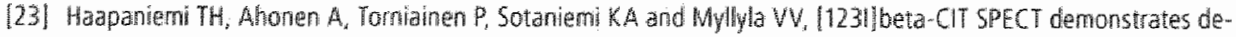
creased braitr dopamine and serotonin transporter levels in untreated parkinsonian patients, Mov Disord, 2001 $16(1) 124 \cdot 130$.

1241 Leentijens AF, Depression in Parkinson's disease: conceptual íssties and dinical challenges, J Geriatr Psychiatry Neurol, 200417 (3) 120-126.

[25) Menza MA. Psychiatric Aspecis of Parkinson's Disease, Psychiatric Ann, 200232 22199-104.

[26] Shulman LM, Taback RL. Bean J and Weiner WJ, Camorbidity of the nonmotor symptoms of Parkinson's disease. Mou Disord, 200116 (3) 507-510.

127 Tandberg E, Larsen IP. Aarsland D. Laake K and Cummings Jl, Risk factors for depression in Parkinson disease, Arch Neurol, $199754(5) 625-630$.

(28) Tom T and Cummings IL, Depression in Parkinson's disease. Phamacological characteristics and treatment, Drugs Aging, 1998 . 12 (1) $55-74$.

(29) Mayeux R, Stern Y, Cote $L$ and Willams JB, Altered serotonin metabolism in depressed patients with parkinson's disease, Neurology, $198434(5) 642-646$

(30) Mayeux $R$, The "serotonin hypothesis" for depression in Parkinson"s disease. Adv Neurol, 199053 163-166.

[31) Van Praag HM and De Haan S. Central serotonin metabolism and frequency of depression, Psychiatry Res, 1979 1219-224.

1321 Orth M and Tabrizi 51, Models of Parkinson's Disease, Mow Disord, $200318(7) 729-737$.

133] Dauer W and Przedborksi S, Parkinson's Disease: Mechanisms and Models, Neuron, $200339889-909$.

[34] Collier TJ, Steece-Collier K and Kordower HH, Primate models of Parkinson's disease, Exp Neurol, 2003 183 (2) $258-262$.

135] Deumens R, Blokland A and Prickaerts J, Madeling Parkinson's Disease in Rats: An Evaluation of 6-OHDA Lesions of the Nigrostriatal Pathway, Exp Neurol, 2002 175(2) 303-317.

(36) Eslamboli A, Baker H, Ridley RM and Annett $L E_{*}$, Sensormotor deficits in a unilateral intrastriatal 6-OHDA partial hesion madel of Parkinson's disease in marmoset monkeys, Exp Neurol, $2003183418-429$.

137/ Tamas A, Lubics A, Szulontay L, Lengvaril and Reglodi D, Age and gender differences in behavioral and morphological outcome after 6-hydroxydopamine-induced lesion of the substantia nigra in rats, Behav Brain Res, 2005 $158(2) 221-229$.

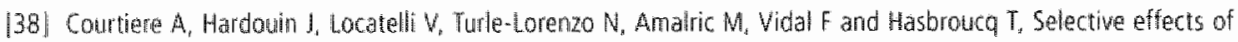
partial striatal 6-OHEA lesions on information processing in the rat, Eur J Neurosci, 200521 (7) 1973-1983.

139| Harrison 8I, Olver 15, Norman TR, Burrows GD, Wesnes KA and Nathan PJ, Selective effects of acute serotonin and catecholamine depletion on memory in healthy women, J Psychopharmacol, 200418 (1) 3240.

[40] Hughes $\mathrm{JH}_{\text {, Gallagher }}$, Stewart ME, Mathews D, Kelly TP and Young $A H_{*}$. The effects of acute tryptophan depletion on neuropsychological function, I Psychophamacol, 200317 (3) 300-309.

[41] Riedel WJ, Klaassen T, Deutz NE. van Soneren A and van Praag HM. Tryptophan depletion in nomal wolunteers produces selective impairment in memory consolidation, Psychopharmacology (Berl), 1999141 (4) 362-369.

1421 Riedel WJ. Klaassen T and Schmit IA, Tryptophan, mood, and cognitive function, Braili Behaw Immun, 200216 (5) $581-589$

143. Riledel W, Cognitive changes after acute tryptophan depletion: what can they tell us?, Psychol Med, 200434 (1) 3-8. 


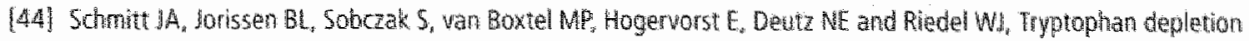
impairs memory consolidation but improves focussed attention in thealthy young wolunteers, I. Psychopharmacol. $2000141112 \%-29$.

[45] Sobczak S. Honig A, Nicolson NA and Rhedel W, Elfects of acute tryptophan depletion on mood and contisol re. lease in first-degree relatives of type land type il bipolat patents and healthy matched controls, Neuropsychopharmacology, $200227(5) 834.842$.

[46] Jacobs BL and Formal CA, 5-HT and motar control: a hypothesis, Trends Neurose;, 1993 16 (9) 346-352.

[47] Ugedo L. Grenhoff J and Svensson TH, Ritanserin, a 5-HT2 receptor antagonist, activates midbrain dopamine neurons by blocking serotonergic inhibition, Psychopharmacology (Berl), 198998 (1) 45-50

[48] Harmer CI, Bhagwagar Z, Cowen PI and Goodwin GM, Acute administration of citalopram facilitates memory consolidation in healthy volunteers, Psychopharmacology (Berl), 2002163 (1) 106-110.

[49] Siepmann $M$, Grossmann s, Muck-Weymann $M$ and Kirch $W$. Effects of sertraline on autonomic and cognitive functions in healthy volunteers. Psychopharmacology (Benl), 2003 168 (3) 293-298.

[50] Cenci MA, Whishaw $1 \mathrm{Q}$ and Schallert T, Animal models of neurological deficits: how relevant is the rat? Nat Rew Netrosci, $20023(7) 574-579$.

[51] Blanchand W, Anglade P, Dziewczapolski G, Savasta M, Agid Y and Raisman-Vozari R, Dopaninergic sprouting in the rat striatum after partial lesion of the substantia nigra, Brain Res, $1996709(2) 399-325$.

[52] Rybicki BA, Johnson CC and Gorell IM, Demographic differences in referral rates to neurologists of patients with suspected Parkinson's disease: implications for case-control study design, 1995:4/ (2) 72-81.

[53) Hughes A., Daniel SE, Kifford $L$ and Lees AJ. Accuracy of dinical diagnosis of idiopathic Parkinson's disease: a dinico pathological study of 100 cases., Neurol Neurosurg Psychiatry, 199255 (3) 181-184.

154) Parkinson J. An assay on the Shaking Paisy, London: Sherwood, Neely and Jones, 1817.

[55] Weintraub $D_{*}$ Moberg $P J_{\text {, Duda }} \mathrm{E}_{\text {, Katz }} \mathrm{R}$ and Stern MB, Effect of psychiatric and other normotor symptoms on disability in Parkinson's disease, 2004.52 (5) 784.788.

[56] Emre $M$, Aarsland $D_{s}$ Albanese A, Byrne Ed, Deuschl $G$, De Deyn PP, Durif $F$. Kulisevsky 1, van Laar $T_{n}$ Lees $A_{r}$ Poewe W, Rabillard A, Rosa MM, Wolters E, Quarg P, Tekin S and Lane R, Rivastignine for dementia associated with Parkinson's disease, 2004351 (24) 2509-2518.

[57] Birkmayer W and Birkmayer JD, Dopamine action and disorders of neuratransmitter balance, Gerontology, 1987 $33(3-4) 168-171$

158) Riederer $P$ and Birkmayer W. A New Concept: Brain Area Specific Imbalance of Neurotoransmitters in Depression Syndrome - Human Brain Studies. In: Usdin En Sourkes TL and Youdim MBH (Eds.), Enzymes and Neurotransmitters in Mental Disease, John Wiley \& Sons. Ltd. 1980, $261-297$.

159| Weintraub D, Newberg AB, Cary MS, Siderow AD, Moberg PJ, Kleiner Fisman G, Duda JE stem MB, Mozley D and Katz IR, Striatal dopamine transporter imaging correlates with anxety and depression symptoms in ParkinSon's disease, J Nucl Med, $200546(2) 227-232$.

[60] de la Fuente-Fernandez $R$ and Stoessl A], The biochemical bases of the placebo effect, 200410 (1) 143-150.

[61] de la Fuente-Fernandez $R$ and Stoessl AJ, The placebo effect in Parkinson's disease, Trends Neurosei, 200225 (6) $302-306$.

[62] de la Fuente-Fernandez R, Ruth TI, Sossi V, Schulzer M, Calne DB and Stoess AJ. Expectation and dopamine release: mechanism of the placebo effect in Parkinson's disease, Science, 2001293 (5532) 1164-1166.

4631 Starkstein SE, Petracca G, Chemerinski E. Teson A, Sabe L, Merello $M$ and Leiguarda $R$, Depression in classic versus akinetic-rigid Parkinson's disease, Wow Disord, 199813 (1) 29.33. 


\section{Summary}

Parkinson's disease (PD) is one of the most prevalent neuropsychiatric diseases. PD has long been considered a pure motor disease, with degeneration of dopaminergic fibers as its main cause. Only recently, it has been recognized that more neurotransmitter sys-

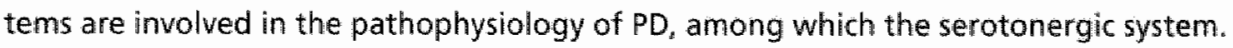
The experiments described in this thesis focus on the role of serotonin in PD. In the first part of this thesis preclinical investigations into the role of serotonin in PD are described. The second part focuses on clinical investigations of this topic.

In chapter 2 we reviewed available literature focusing on functional interventions of the serotonergic neurotransmitter system in PD. Results from both animal research and human research were discussed. It was found that data from both fields of research yielded contrary results. Animal models seem to support the hypothesis stating that a reduced serotonin level forms a compensation for the reduced dopamine level, while studies in humans do not appear to support this hypothesis.

In spite of this disparity, it remains important to combine preclinical and clinical research in order to gain more knowledge about basic mechanisms and interactions be. tween neurotransmitter systems and develop a possible cure for PD. The disparity between both fields of research has to be taken into account when combining findings from clinical and predinical research, since a one on one comparison remains unfea. sible.

In chapter 3 we used an animal model for PD, the partial bilateral striatal 6-hydroxydopamine (6-OHDA) model. This model was used in rats, which were behaviorally tested. Moreover, the effects of different serotonergic and dopaminergic substances on the motor behavior of these rats were investigated. Results showed that serotonin and dopamine appear to be agonistically linked to each other in both 6-OHDA lesioned animals as well as in control animals. Furthermore, we observed a behaviloral recovery of function at approximately 12 weeks post lesion. This behavioral recovery implies that when using this model, a specific time frame (i.e. the first two months post lesion' has. 
to be taken into account for behavioral testing, and that the 6-OHDA model loses its usefulness for investigating $\mathrm{PD}$ symptoms after this period.

In chapter 4 we described that the recovery which was observed during behavioral testing of the animals in chapter 3 was paralleled by an anatomical recovery of the 6-OHDA lesioned Caudate Putamen complex ( $\mathrm{CPu}$, corresponding to the striatum in humans). This was visualized by tyrosine hydroxylase (TH) staining of the lesioned striatal area. This observation once more stresses the importance of the time frame which has to be taken into account when using the 6-OHDA lesion model.

The results of the Acute Tryptophan Depletion (ATD) experiment (the first part of two complementary experiments) were discussed in chapters $5 \& 6$. In chapter 5 the effects of ATD on aspects of motor functioning and cognition were discussed. Results showed that acutely lowering serotonergic availability in PD patients and control subjects did not have differential effects in both groups. Although acutely lowering serotonergic availability did have a positive effect on reaction time responding (in both patients and controls), this effect was not considered to be clinically relevant.

In chapter 6 we tested the serotonergic hypothesis for depression in PD using an experimental approach. This hypothesis states that the reduction of serotonin is a compensation mechanism for the degeneration of the dopaminergic system. Results indicated that acutely intervening with serotonergic availability by means of ATD had no specific effects on mood state and cortisol response. The findings of this experimental approach provide no evidence to support the serotonergic hypothesis for depression in $\mathrm{PD}$, and open the way for alternative hypotheses.

Chapter 7 described the results of the second part of the clinical experiments. In the present experiment, we acutely challenged the serotonergic system in a specific and aspecific manner, using a 5-HT 1 a agonist and a SSRI. Results indicated that receptor specific and aspecific challenge of the serotonergic neurotransmitter system had comparable effects in both PD patients and control subjects. Furthermore, an impairment in performance after challenging the serotonergic system was visible in the tasks measuring reaction time responding. These observations are complementary to the results of the ATD experiment described in chapter 3.

In chapter 8 we described results of a newly developed task, the Manual Tracking Task for Tremor Assessment (MTT-TA). We attempted to develop a task which could easilly be used in both a clinical and experimental setting providing clinicians and researchers with information about the ability of PD patients to perform simple manual tracking 
movements and detect the severity of a possible tremor. This new task was part of the test battery of both the ATD and the challenge study, described in chapters 5,6 , and 7. Results described in chapter 8 described the effects of ATD on performance of the MTT-TA. Results showed that ATD had a beneficial effect on the execution of simple manual tracking movement. This implies that the MTT-TA is sensitive to medication induced changes in performance. We did not observe any baseline differences in performance on the MTT-TA between PD patients and control subjects, which may reflect a limited discriminant validity. Another possible explanation for the absence of the baseline difference between patients and control subjects might be that the severity of the disease of the patients was not large enough. This, however, does not mean that the task should not be used in further experiments. In its present form, the MTT-TA can be of use in monitoring therapeutic effects, but may be less suited as a diagnostic instrument. Slight alterations of the task may also improve its discriminant validity. Validation of the MTT-TA with respect to other methods for assessing aspects of movement and tremor in PD patients has yet to be performed.

In chapter 9 all results presented in this thesis were brought together and discussed. Results showed that findings from preclinical and clinical research are not always in agreement with each other. Furthermore, serotonin still appears to be functioning in an adequate way in patients with mild PD, and no evidence in support of the serotonergic hypothesis for depression in PD was found. Methodological issues and clinical implications are discussed, and recommendations for future research are given. 


\section{Samenvatting}

De ziekte van Parkinson (PD) is een van de meest prevalente neuropsychiatrische ziektebeelden. Lange tijd werd PD gezien als een 'pure' motorische aandoening met de degeneratie van dopaminerge vezelbanen als de voornaamste oorzaak. Recentelilk is duidelijk geworden dat meerdere neurotransmittersystemen betrokken zijn bij de pathofysiologie van PD. Een van deze andere betrokken neurotransmittersystemen is het serotonerge systeem. De experimenten die in deze thesis beschreven zijn richten zich op de rol van serotonine in PD. In het eerste gedeelte van deze thesis wordt aandacht geschonken aan twee preklinische experimenten naar de rol van serotonine in PD. Het tweede gedeelte van deze thesis richt zich op de klinische experimenten die deel uitmaken van dit promatietraject.

In hoofdstuk 2 werd de bestaande literatuur omtrent functionele interventies van het serotonerge neurotransmitter systeem beschreven. Resultaten uit zowel dierexperimenteel (preklinisch) alsook klinisch onderzoek worden besproken. Er werd gevonden dat data uit beide onderzoeksvelden tegengestelde resultaten oplevert. Resultaten uit dierexperimenteel onderzoek lijken de hypothese te ondersteunen die stelt dat een reductie in serotonerge niveaus een compensatie mechanisme vormt voor de afgeno. men dopaminerge niveaus. Klinische studies lijken deze hypothese niet te ondersteunen. Ondanks deze discrepantie blijft het belangrijk preklinisch en klinisch onderzoek te combineren. Op deze manier kan meer kennis verworven worden over basale mechanismen en interacties tussen werschillende neurotransmitter systemen, hetgeen kan leiden tot een betere behandeling van, en mogelijke genezing voor PD. De discrepantie tussen beide onderzoeksgebieden moet echter wel in acht genomen worden wanneer resultaten van klinisch en preklinisch onderzoek gecombineerd worden. Een 'een op een' vergelijking blijft moeilijk, zoniet onmogelijk.

In hoofdstuk 3 werd gebruik gemaakt van een diermodel woor PD, het zogenaamde "partial bilateral striatal 6-hydroxydopamine (6-OHDA) model". In dit model werd gebruik gemaakt van ratten als proefdier. Deze dieren werden gedragsmatig getest. Ver- 
der werden de effecten wan verschillende serotonerge en dopaminerge stoffen op het motorisch gedrag van de dieren getest. De resultaten van dit onderzoek lieten zien dat serotonine en dopamine agonistisch aan elkaar gelinkt te zijn in zowel de 6-OHDA gelaedeerde dieren alsook in de controle dieren. Verder werd herstel in het gedrag van de dieren geobserveerd 12 weken nad at de operaties uitgevoerd waren. Dit herstel van functie geeft aan dat wanneer dit model gebruikt wordt, men rekening dient te houden met de specifieke periode ( 2 maanden na operatie) waarbinnen het model een goede representatie van de PD-symptomen representeert. Na deze periode verliest het model zijn bruikbaarheid om PD-symptomen te onderzoeken.

In hoofdstuk 4 werd het in hoofdstuk 3 beschreven herstel van functie verder onderbouwd door de observatie van de aanwezigheid van anatomisch herstel van het Caudate Putamen complex (het $\mathrm{CPu}$, striatum bij de mens). Dit werd gevisualiseerd door de microscopische bestudering van tyrosine hydroxylase $(\mathrm{TH})$ gekleurde coupes van het geleadeerde striatale gebied. Deze observatie benadrukt eens te meer het belang van het in acht nemen van de specifieke tijdsperiode wanneer gebruik wordt gemaakt van het eerder beschreven 6-OHDA model voor PD.

De resultaten van het Acute Tryptofaan Depletie (ATD) onderzoek (het eerste deel van twee complementaire experimenten) werden bediscussieerd in hoofdstukken $5 \& 6$. In hoofdstuk 5 werden de effecten van ATD op aspecten van motorisch functioneren en cognitief functioneren onderzocht. Resultaten lieten zien dat het acuut verlagen van serotonerge beschikbaarheid in PD patiënten en gezonde controle personen dezelfde effecten had in beide groepen. Alhoewel het acuut verlagen van serotonine niveaus een positief effect had op de prestatie op reactietijdtaken (zowel in patiënten als in controles), werd dit effect niet als klinisch relevant beschouwd.

In hoofdstuk 6 werd de serotonerge hypothese voor depressie bij PD met behulp van een experimentele benadering getest. Deze hypothese stelt dat de afname van serotonine bij PD patiënten een compenserend mechanisme is voor de aanwezige degeneratie van het dopaminerge systeem. Het acuut verlagen van serotonerge activiteit, door middel van de ATD methode, had geen specifieke effecten met betrekking tot stemming en cortisolactiviteit. De bevindingen van deze experimentele aanpak bieden geen bewijs voor de serotonerge hypothese voor depressie bij PD. Deze bevindingen openen wel de weg voor het experimenteel onderzoeken van alternatieve hypotheses.

Hoofdstuk 7 beschreef de resultaten van het tweede deel van de klinische experimenten. Hier werd het serotonerge systeem op een specifieke en aspecifieke manier, met behulp van een $5-\mathrm{HT}_{1 \mathrm{a}}$ agonist en een selectieve serotonine heropname remmer (SSRI) 
gestimuleerd. Uit dit onderzoek kwam naar voren dat specifieke en aspecifieke stimulatie van het serotonerge neurotransmittersysteem vergelijkbare effecten hadden in zowel PD patiënten als controles. Verder bleek dat de serotonerge stimulatie een negatief effect had op de prestatie op reactietijd taken. Deze abservatie is tegengesteld aan de resultaten van de ATD interventie methode beschreven in hoofdstuk 3.

In hoofdstuk 8 werden de resultaten van een nieuwe taak, de Manual Tracking Task for Tremor Assessment (MTT-TA) beschreven. Deze taak werd ontwikkeld om op een makkelijke manier in zowel een klinische als een experimentele setting informatie te verschaffen over de mate waarin PD-patiënten gestoord zijn in het maken van zogenaamde 'simple manual tracking movements'. Tevens was het de bedoeling met de MTT-TA een objectieve indruk te krijgen van de aanwezigheid en mogelijke ernst van een tremor. Deze nieuwe taak maakte deel uit van de testbatterij die gebruikt werd in de ATD en challenge studies, beschreven in hoofdstukken 5, 6, en 7. De resultaten in hoofdstuk 8 beschrijven de effecten van ATD op de prestatie van PD-patiènten en controles op de MTT-TA. ATD had een positief effect op de uitvoering van 'simple manual tracking movements'. Dit betekent dat de MTT-TA gevoelig is voor, door farmacologische interventies geïnduceerde, veranderingen in de prestatie op deze taak. Er werden geen verschillen tussen de patiënten en de controles waargenomen op de baselinemeting. Dit weerspiegelt mogelijk een beperkte discriminerende validiteit. Een andere mogelijke verklaring voor de afwezigheid van een baselineverschil tussen beide groepen ligt mogelijk in het feit dat de meeste geïncludeerde patiënten een milde vorm van de ziekte van Parkinson hadden, wat inhoud dat de motorische symptomen nog niet zeer ernstig waren. Dit betekent echter niet dat de MTT-TA niet meer gebruikt dient te worden in volgende experimenten. In zijn huidige vorm is de MTT-TA geschikt voor het volgen van therapeutische effecten, maar is niet geschikt als diagnostisch instrument. Kleine ver* anderingen in de taak kunnen resulteren in een verbeterde discriminerende validiteit. Validatie van de MTT-TA ten opzichte van andere tests voor het meten van bewegingen en tremor moet nog plaatsvinden.

In hoofdstuk 9 werden alle in deze thesis gepresenteerde resultaten samengebracht en bediscussieerd. Bevindingen uit preklinisch en klinisch onderzoek bleken niet altijd in overeenstemming met elkaar te zijn. Verder functioneert het serotonerge systeem in de geteste PD-patiënten nog adequaat. Er werd geen bewijs gevonden voor de serotonerge hypothese voor depressie bij Parkinson. Methodologilische zaken en klinische implicaties worden besproken, en aanbevelingen voor toekomstig onderzoek worden gegeven. 


\section{Dankwoord}

Het laatste, en waarschijnlijk meest gelezen, 'hoofdstuk' van het proefschrift is misschien ook wel het meest lastige om te schrijven. Het onderzoek waaruit dit proefschrift is ontstaan had natuurlijk niet uitgevoerd kunnen worden zonder de hulp van velen die de afgelopen jaren, al dan niet vanaf de zijlijn of in het heetst van de strijd. hun steentje (kiezel of kei) aan de totstandkoming van het proefschrift hebben bijgedragen. Het is al vaker gezegd, een proefschrift schrijf je niet alleen...

Als eerste wil ik graag alle deelnemers bedanken die aan dit onderzoek hebben meege* daan: zonder jullie zou patientgebonden onderzoek ophouden te bestaan! En zonder jullie medewerking was dit boekje nooit afgekomen.

Dan de "harde kern" van het onderzoeksteam. Als eerste Prof. dr. Verhey, mijn eerste promotor. Frans, je was niet altijd 'direct' betrokken bifi mijn dagelijkse begeleiding, maar was echter steeds op de achtergrond, en in het laatste jaar cok steeds meer op de voorgrond aanwezig om mij waar nodig met raad en daad te helpen bij mijn ontwikkeling tot 'zelfstandig wetenschapper'. Ik wil je bedanken voor je steun en je ongelimiteerde vertrouwen in de goede afloop van het onderzoek en mijn functioneren! $\mathrm{k}$ heb dit altijd zeer gewaardeerd. Het onderzoek bespreken met andere AlO's en collega's. onder het genot van een drankje en een overheerlijke BBQ in 'sjoen' Bemelen zal ik ook. zeker niet snel vergeten, en het zou leuk zijn om hier een traditie van te maken.

Mijn tweede promotor, Prof. dr. Steinbusch. Beste Harry, bedankt voor de ruimte en mogelijkheid die ik gekregen heb voor het uitwoeren van de preklinische experimenten. Ik heb er veel van geleerd! Ik denk dat het ook zeer belangrijk is om in de toekomst beide onderzoekstakken te blijven combineren, ook al is dit niet altijd een mak. kelijke weg gebleken.

Dr. Leentjens, co-promotor. Beste Albert, ik wil je bedanken woor de wekelijkse begleleiding. Van je gestructureerde, nauwgezette werkwijze en je theoretische kennis heb ik vaak staan kijken, maar hier heb ik zeker ook veel van geleerd! En tijdens onze gezamenlijke reis naar Salzburg heb ik je van een andere kant leren kennen. Het diner en de halve liters witbier met Wim in die Oostenrijkse Stube, staan in mijn geheugen gegrift... 
Dr. Vreeling, co-promotor. Beste Fred, door jou heb ik geleerd wat de ziekte van Parkinson echt is, en wat het betekent voor de patiënten en hun familie. In de uren die ik met je op de poli Neurologie heb mogen doorbrengen heb ik enorm veel geleerd over de ziekte van Parkinsom en de behandeling ervan. Aan jouw kijk op het vak neuroloog (en het leven) zouden veel artsen een voorbeeld mogen nemen. Dank je wel dat ik een kijkje in je keuken heb mogen nemen! Ik hoop dat we elkaar de komende jaren zeker nog eens tegen zullen komen.

Voorafgaand aan een promotietraject heb je natuurlijk een goede stageplaats nodig. Die vond ik bij Arjan Blokland. Arjan, van jou heb ik tijdens mijn stage de magelijkheid gekregen om een kijkje te nemen in de dierexperimentele wereld. Jouw passie om als biologisch psycholoog met gedrag bezig zijn en hersenen te onderzoeken, heeft ook mij aangestoken om verder te gaan met onderzoek. Dank je wel voor all die leerzame en leuke momenten, ik heb enorm veel van je geleerd!! Ook je betrokkenheid bij de voortgang wan mijn AlO project heb ik altijd zeer gewaardeerd. En het gedicht dat je een kleine vijf jaar geleden voordroeg is nog steeds van toepassing...

Naast de samenwerking met de afdelingen neurologie en basale neurowetenschappen was er ook de samenwerking met de afdeling bewegingswetenschappen. In het bijzonder wil ik hier Jos Adam bedanken. Beste Jos, bedankt voor de openhartige manier waarop je me kennis helot laten maken met de wondere wereld van de functieleer. De talrijke (basaal) wetenschappelijke discussies bij het bespreken van artikelen waar het enthousiasme wel eens vanaf vloog zal ik me nog lang herinneren. Ik heb me altijd zeer welkom gevoeld bij jullie op het lab, en heb er ook zeker veel van geleerd. Ik hoop dat we ellkaar in de toekomst ook nog tegen zullen komen en mogelijk ook nog eens kunnen samenwerken!

Wim Weber en Dr. P. Koehler will ik danken voor de 'verdere neurolagische samenwerking". Wim bedankt woor de leuke momenten op de gang (we waren immers ooit buren) en de samenwerking binnen de nieuwe 'poli bewegingsstoornissen'. Beste Dr. Koehler, bedankt voor de hulp bij het vinden van nieuwe patiënten toen de "voorraad" in Maastipht ten einde $\mathrm{kwam}$. lk waardeer het zeer dat u lid wilde zijn van de beoordelingscommissie van mijn proefschrift.

Een promotie kan natuurlijk niet plaats vinden zonder de steun van twee goede paranimfen (al was het maar om eventueel een vraag door te kunnen spelen〉.

Beste Jas, misschien realiseer je het niet, maar jij bent min of meer schuld aan het feit dat ik het onderzoek ben ingegaan. Jij was immer degene die mijn interesse in het wetenschappelijk onderzoek pas echt heeft aangewakkerd! De rondleiding die ik van 
je kreeg na afloop van een practicum bij psychologie was voor mij reden genoeg om meer te willen weten over die, tot dan toe, onbekende onderzoekswereld. Je bent voor mij een soort Yoda, een bijna onuitputtelijke bron van kennis en enthousiasme (zowel op de werkvloer als thuis...). In eerste instantie maakte je ook deel uit van 'de harde kern', maar later is daar helaas een eind aan gekomen toen je van baan veranderde. Ik heb je toen meteen gevraagd of je mijn paranimf wilde worden. Ik ben dan ook zeer vereerd dat je die wraag met een volmondig 'ja' beantwoord hebt! En nog mooier vind ik dat er uit onze werkrelatie ook een vriendschappelijke band is ontstaan (samen met Jeanette en de kids natuurlijk), met als hoogtepunt: de wonderbaarlijke avonturen in een bootje onder de Pont d'Arc in de zon in Frankrijk!

Beste Josien, mede BAJO-partner, we zaten ongeveer op gelijke hoogte in het promotieproces (al begon je later en promoveer je ook nog eens twee weken eerder...). Hierdoor konden we wanneer nodig even stoom af blazen over allerlei (vast triviale) problemen die een AlO tegen kan komen... Gelukkig konden we onze spanning ook kwijt in het Sportdomein (of had het iets met angstzweet te maken?) en natuurlijk via de mail. Ik vind het erg leuk, en voel me vereerd, dat we onafhankelijk van elkaar besloten hebben om elkaar voor de rol van paranimf te vragen! lk hoop dat we in de toekomst nog vaak gezellige etentjes zullen hebben (natuurlijk samen met Steven en Dymphie), al dan niet vergezeld van de albums met foto's die je telkens van je verre en soms ook gevaarlijke reizen mee terug neemt. Dank voor je steun en vriendschap!!

Alhoewel het AlO-bestaan vaak eenzaam kan zijn, zit je gelukkig zelden alleen op je kamer. Ik heb de afgelopen jaren een kamer mogen delen met een aantal mensen. Het begon allemaal op een kamer met Tessa Kilkens en Marieke Wichers. Ongeveer op hetzelfde moment begonnen we aan ons promotieproject. We hebben de $21 / 2$ jaar dat we een kamer gedeeld hebben veel gelachen en ook hard gewerkt (de een wat harder dan de ander...). Marieke, je bent al llang en breed gepromoveerd, en Tessa, jij heel veel succes met je eigen promotie en verdere opleiding tot arts. Bedankt voor de gezellige tijd op de kamer met het mooiste uitzicht van de gang!

In een dynamisch bedrijf moet natuurlijk ook eens van kamer gewisseld worden, dus na 21/2 jaar was het tijd om te verhuizen en kwam ik bij Petra Kuijpers op de kamer terecht. Petra, ook al was jij vaak niet lijfelijk op onze kamer aanwezig omdat je druk bezig was met andere belangrijke zaken (je bent immers een beroemd cardioloog), jij was ongetwijfeld mijn gezelligste kamergenoot (al is deze uitspraak ondertussen niet meer echt origineel...). Ondertussen hebben we al menig maal van elkaars kookkunsten mogen genieten, en ik hoop dat we dat de komende jaren ook zullen blijven doen!

Ook aan de tijd met Petra kwam een eind, want bij de volgende verhuizing kreeg ik weer een nieuwe kamergenoot, Pauline Aalten. Bij jou klikte het eigenlijk ook meteen. Naast de nodige wetenschappelijke discussies heb ik het ook altijd erg gezellig en 
leuk gevonden om het te hebben over al die andere dingen die het leven leuk, en soms ook minder leuk maken...

Naast de mensen met wie ik een kamer heb mogen delen waren er natuurlijk ook een hoop andere mensen die het werk de afgelopen jaren wan de nodige afwisseling hebben voorzien en altijd wel in waren voor een (kort of wat langer) praatje. Met name Jeanette Dijkstra, Femke Dings, Floor van Bergen, Sandra Rosmuller, Inez Ramakers, Ilse Janssen, en Jeroen Schmitt (ook al zit je ondertussen vele honderden kilometers verder. op in Zwitserland) bedankt voor de vele gezellige en leerzame momenten!

Carla Brandts, jou wil ik hier speciaal bedanken woor je kunstzinnige kijk op het leven en de bijzondere kaft van het proefschrift. Ik ben er nog steeds erg van onder de indruk. Ik hoop dat ik je nog vaak in verlegenheid en aan het lachen mag brengen, en dat iij dit ook bij mij zult blijven doen...

En dan zijn er natuurlijk nog vele andere collega's die ik hier nu niet bij naam noem (want dan kan ik eigenlijk wel iedereen op de gang gaan opnoemen), maar die het verblijf de afgelopen jaren zeker ook veraangenaamd hebben, dank jullie allemaal voor de leuke en gezellige momenten!

Naast de collega's op de gang bij Neuropsychologie heb ik ook de nodige tijd in het lab op de UNS50 doorgebracht. Daar wil ik vooral Wiel (tondeuse) Honig, Ronall (sjnaps du dat noe neet?) Deumens, en Guido (kano) Koopmans bedanken met wie ik vele gezellige en zeker ook wetenschappelijk verantwoorde en leerzame uren heb doorgebracht. Jongens bedankt woor deze leuke tijd! Ook Hellen Steinbusch wil ik bedanken voor de prettige samenwerking en de leerzame momenten op het lab! Christoph Schmitz, herzlichen Dank für deine Mühe bei dem Verarbeiten der mikroskopischen Bilder.

Ook bij de meer praktische kant van het werk heb ik er gelukkig niet altijd alleen voor gestaan. Jennifer, dank je wel voor je enthousiaste inzet bij de dataverzameling en invoer! Je was telkens bereid om bij te springen, ook al had je eigenlijk al meer dan genoeg gedaan om je wetenschapsstage af te ronden. Zonder jouw hulp was ik vast en zeker nu nog steeds patiënten aan het testen!

Voor de lay-out van het proefschrift wil ik Anita Kaemingk danken. Anita, dank je wel voor al het werk dat je in m'n proefschrift gestoken hebt! Het ziet er allemaal prachtig uit! Toen ik die vrijdag voor de eerste keer uitleg van je kreeg over hoe je een boekwerk mooi in elkaar zet, ging er voor mij een nieuwe wereld open. Ik heb die hele ochtend met $m$ "n oren zitten flapperen, maar heb ook hier erg veel wan geleerd. Het resul-m taat mag er wat mij betreft zijn!! Diezelfde dag werd ook de lay-out van de kaft onder handen genomen. Ans Dirx en Pieter Spruyt, bedankt voor jullie creatieve ideeën. 
Naast alle collega's is er natuurlijk ook een thuisfront en famlie waar je van tijd tot tijd je hart kunt luchten en soms eens uit moet leggen waar je nu eigenlijk mee bezig bent. Vragen zoals: "ben je nu nog steeds aan het studeren?", "wanneer krijg je je diploma nu?" en "je bent toch bezig met onderzoek naar de ziekte van Alzheimer?!" werden wel eens gesteld... (niet door iedereen overigens). Lieve Fia, Karel Joep, Opa, Oma, Truus, Ghis en Etienne, bedankt voor de steum en interesse waar ik altijd op kon en kan rekenen!!

Naast familie zijn er ook nog een aantal bijzondere wrienden waar ik altijd welkom was om onder het genot van een hapje en een drankje te kunnen relaxen en tijdelijk te ontsnappen aan het werk van de afgelopen tijd. Veronique en Sven, Desiree en Fer, Jeanette en Jos, Josien en Steven, ik hoop dat we in de toekomst nog vele leuke momenten met elkaar zullen belleven!!

En tenslotte natuurlijk Dymphie. Elke dag ben ik weer blij dat ik ooit tegen je aan ben geschaatst... Dank je wel voor je onvoorwaardelijke liefde en steun. Het leven met jou is gewoon geweldig!!!!

Maastricht, september 2005.

Bart 



\section{Publications}

- A. Blokland, B. Scholtissen, A. Vermeeren, and I. Ramaekers Dissociable effects of histamine H'l antagonists an reaction-time performance in rats Pharmacology Biochemistry and Behavior. 2001 70(2-3);427-436.

- Y. Temel, V. Visser-Vandewalle, B. Aandekerk, B. Rutten, 5. Tan, B. Scholtissen ${ }_{i}$ C. Schmitz, A. Blokland, and H.W.M. Steinbusch Dissaciation of motor and cognitive processing in parkinsonian rats during bilateral subthalamic nucleus stimulation

Exp Neurol. 2005 May:193(1):43-52.

- B. Scholtissen, F.R.J. Verhey, H.W.M. Steinbusch, and A.F.G. Leentjens Serotonergic mechanisms in Parkinson's Disease: Opposing results from predinical and clinical data Journal of Neural Transmission (accepted).

- B. Scholtissen, F.R.J. Verhey, J.J. Adam, J. Prickalerts, and A.F.G. Leentjens Effects of acute tryptophan depletion on cognition, memory and motor performance flournal of the Neurological Sciences (accepted).

- B. Scholtissen, J. Dijkstra, J. Reithler, and A.F.G. Leentjens Verbal fluency is not impaired" in patients with Parkinson's disease Acta Neuropsychiatrica (accepted)

- A.F.G. Leentjens, B.Scholtissem, F. Vreeling, and F.R.J. Verhey No evidence for the serotonergic hypothesis of depression in Parkinson's disease in an experimental approach Neuropsychopharmacology (accepted)

- B. Scholtissen, R. Deumens, A.F.G. Leentjens, C. Schmitz, A. Blokland, H.W.M. Steinbusch, and $\mathrm{J}$. Prickaerts.

Functional investigations into the role of dopamine and serotonin in partial bilateral striatal 6-hydroxydopamine lesioned rats Pharmacology, Biochemistry and Behaviar (under revision)

- B. Scholtissen, F.R.J. Verhey, J. J. Adam, and A.F.G. Leentjens Challenging the serotonergic system in Parkinson's disease patients:

Effects on cognition, memory, motor performance and mood submitted 



\section{Curriculum Vitae}

Bart Scholtissen werd geborein op 7 januari 1978 in Heerlen. Na het behalen van zijn VWO diploma aan het Bernardinus college in Heerlen (in 1996) werhuisde hij naar Maas. tricht en volgde hij de studie psychologie aan de Universiteit Maastricht. De afsluitende wetenschapsstage ging over de rol van serotonine bij de ziekte van Parkinson, en stond onder begeleiding stond van Dr. A. Blokland en Dr. A.F.G. Leentjens. Ook was hil als student-assistent betrokken bij twee wetenschappelijke projecten van de vakgroepen Neonatologie en Psychologie. In 2000 studeerde hij af en werd hij aangesteld als Assistent In Opleiding (AIO) bij de vakgroep Psychiatrie en Neuropsychologie. Het project had als doel meer inzicht verkrijgen in de rol van serotonine bij de ziekte van Parkinson. Aangezien het handelde om een zogenaamd Brede Onderzoek Strategle (BOS) AIO-project betekende dit dat hij werkzaam was bij zowel de vakgroep Basale Neurowetenschappen alsook de vakgroep Psychiatrie. Dit resulteerde in dit proefschrift. Naast het onderzoek heeft hij ook een onderwijsaanstelling gehad bij de faculteit der Geneeskunde van de Universiteit Maastricht.

Momenteel is hilj werkzaam voor de vakgroep Psychiatrie en Neuropsychologie van de Universiteit Maastricht en houdt hij zich bezig met onderwijs en onderzoek. 